\title{
Singularidades de curvas na Geometria Afim ${ }^{1}$
}

\author{
Luis Florial Espinoza Sánchez
}

Orientador: Prof. Dr. Marcelo José Saia

Dissertação apresentada ao Instituto de Ciências Matemáticas e de Computação - ICMC-USP, como parte dos requisitos para obtenção do título de Mestre em Ciéncias - Matemática.

USP - São Carlos

Agosto / 2010

\footnotetext{
${ }^{1}$ Este trabalho teve suporte financeiro do $\mathrm{CNPq}$
} 



\section{Agradecimentos}

Primeiramente, agradeço a Deus por ter me dado oportunidade de estar aqui, e por sempre guiar meus passos.

Ao professor Marcelo José Saia, os meus agradecimentos, pela sua orientação, motivações, ensinamentos, conselhos que me deu para poder ter desenvolvido esta disertação e pela amizade que sempre me oferece.

À minha mãe pelo apoio que sempre me brindou e por todos os sacrifícios que fez para que eu pudesse continuar estudando. À meu irmão Sergio que sempre me apoia e por ser um bom amigo. Aos meus irmãos Andrea e Ruben que também sempre me apoiam.

À meus amigos José, Nancy, Manuel, Napoleón, Alvaro,... todos eles tem minha gratidão e respeito pois além que são bons amigos, são excelentes estudantes.

À minha namorada Telma, pelo carinho que ela me dá.

Aos professores do ICMC pelo suporte importante em minha formação acadêmica.

Ao $\mathrm{CNPq}$, pelo apoio financeiro, sem o qual não seria possível a realização deste trabalho. 
A minha mãe, Palmira, e aos meus irmãos, minha infinita gratidão, por tudo o que representam em minha vida. 


\section{Resumo}

Neste trabalho estudamos a geometria da evoluta afim e da curva normal afim associada à uma curva plana sem inflexões a partir do tipo de singularidade das funções suporte afim. O principal resultado estabelece que se $\gamma$ é uma curva plana sem inflexões, satisfazendo certas condições genéricas então dois casos podem ocorrer:

1. se $p$ é um ponto da evoluta afim de $\gamma$ em $s_{0}$ então temos dois casos: se $\gamma\left(s_{0}\right)$ é um ponto sextático então, localmente em $p$, a evoluta afim é difeomorfa a uma cúspide em $\mathbb{R}^{2}$; se não, localmente em $p$, a evoluta afim é difeomorfa à uma reta em $\mathbb{R}^{2}$,

2. se $p=\gamma^{\prime \prime}\left(s_{0}\right)$ é um ponto da normal afim de $\gamma$ então temos dois casos: se $\gamma\left(s_{0}\right)$ é um ponto parabólico de $\gamma$ então, localmente em $p$, a curva normal afim é difeomorfa a uma cúspide em $\mathbb{R}^{2}$; em outro caso, localmente em $p$, a curva normal afim é difeomorfa à uma reta em $\mathbb{R}^{2}$. 


\section{Abstract}

In this work we study the geometry of the affine evolute and the affine normal curve associated with a plane curve without inflections from the type of singularity of affine support functions. The main result is setting if $\gamma$ is a flat curve without inflections, satisfying certain conditions generic then, if $\mathrm{p}$ is a point of the affine evolute of $\gamma$ at $s_{0}$ then two cases: if $\gamma\left(s_{0}\right)$ is a sextactic point then locally in $\mathrm{p}$ the affine evolute is diffeomorphic to a cusp at $\mathbb{R}^{2}$, otherwise locally in $\mathrm{p}$ the affine evolute is diffeomorphic to a straight in $\mathbb{R}^{2}$, and second if $p=\gamma^{\prime \prime}\left(s_{0}\right)$ is a point of the affine normal curve then two cases: if $\gamma\left(s_{0}\right)$ is a parabolic point of $\gamma$ then locally in $\mathrm{p}$ the affine normal curve is diffeomorphic to a cusp at $\mathbb{R}^{2}$, in otherwise locally in $\mathrm{p}$ the affine normal curve is diffeomorphic to a line in $\mathbb{R}^{2}$. 


\section{Sumário}

Introdução 1

1 Curvas no espaço Euclidiano 5

1.1 Curva parametrizada regular - comprimento de arco . . . . . . . . . . 5

1.2 Curvatura e equações de Serret-Frenet . . . . . . . . . . . . . . . 8

1.2.1 Curvatura e normal para curvas planas . . . . . . . . . . . . . . 8

1.2.2 Fórmulas de Serret-Frenet para curvas planas . . . . . . . . . . . . 10

1.2.3 Fórmulas de Serret-Frenet para curvas no espaço . . . . . . . . . . 10

1.3 Contato entre curvas . . . . . . . . . . . . . . . . . . . . . 11

1.4 Funções definidas sobre curvas . . . . . . . . . . . . . . . . . . . . 15

2 Sobre Teoria de Singularidades $\quad 17$

2.1 Equivalência a direita . . . . . . . . . . . . . . . . . 17

2.2 Jatos de funções . . . . . . . . . . . . . . . . . . . . . . . . 22

2.3 Desdobramentos . . . . . . . . . . . . . . . . . . 23

2.4 Desdobramento $(\mathrm{p})$ versal . . . . . . . . . . . . . . . . 25

2.4.1 Conjunto singular - conjunto bifurcação . . . . . . . . . . . . . . . 27

2.4.2 Desdobramento (p)versal de $A_{2}(r \geq 1) \ldots \ldots$. . . . . . . 28

2.4.3 Desdobramento (p)versal de $A_{3}(r \geq 2) \ldots \ldots$. . . . . . . 29

2.5 Desdobramento versal . . . . . . . . . . . . . . . . . . 30

2.5.1 Conjunto discriminante . . . . . . . . . . . . . . . . . 31

2.5.2 Desdobramento versal de $A_{1}(r \geq 1) \ldots \ldots . \ldots . \ldots . \ldots 32$

2.5.3 Desdobramento versal de $A_{2}(r \geq 2) \ldots \ldots . \ldots . \ldots 32$

2.6 Conjuntos bifurcação e discriminante . . . . . . . . . . . . . . . . . . . 33 
3 Curvas planas no espaço afim 35

3.1 Geometria Euclidiana - geometria afim . . . . . . . . . . . . 35

3.1.1 Geometria Euclidiana . . . . . . . . . . . . . . . . 36

3.1 .2 Geometria afim . . . . . . . . . . . . . . 37

3.1.3 Transformações afins e cônicas . . . . . . . . . . . . . . . . . . 38

3.2 O espaço afim . . . . . . . . . . . . . . . . . . . . . . 39

3.3 Comprimento de arco afim - curvatura afim . . . . . . . . . . . . . 42

3.3.1 Comprimento de arco afim . . . . . . . . . . . . . . . . 43

3.3.2 Curvatura afim . . . . . . . . . . . . . . . . 46

3.4 Tangentes afins e normais afins . . . . . . . . . . . . . . . . . 49

3.5 Reta tangente afim e reta normal afim . . . . . . . . . . . . . . . 53

3.6 Transformação equiafim . . . . . . . . . . . . . . . . . . . 55

3.7 Funções suporte afim . . . . . . . . . . . . . . . . . . . . . 57

3.7.1 Funções distância ao cubo afim . . . . . . . . . . . . . . . . . . 57

3.7 .2 Função altura afim . . . . . . . . . . . . . . . . . . . . . 61

4 Aberração $\quad 63$

4.1 Interpretação geométrica da primeira derivada . . . . . . . . . . . . . 63

4.2 Interpretação geométrica da segunda derivada . . . . . . . . . . . . . . . . 64

4.3 Interpretação geométrica da terceira derivada . . . . . . . . . . . . . . 65

4.3.1 Raio de aberração . . . . . . . . . . . . . . . . . . . . . . . 68

4.3 .2 Centro de aberração . . . . . . . . . . . . . . . . 69

4.4 Contato de curvas . . . . . . . . . . . . . . . . . . . . . . 70

4.5 Ordem do contato . . . . . . . . . . . . . . . . . 71

4.5.1 Círculo osculador . . . . . . . . . . . . . . . . . 73

4.5.2 Parábola osculadora . . . . . . . . . . . . . . . . . . 73

4.5 .3 Cônica osculadora . . . . . . . . . . . . . . . . 74

4.5.4 Contato de ordem $6 \ldots \ldots \ldots \ldots$. . . . . . . . . . . . . . . . . .

5 Pontos sextático e parabólico $\quad 79$

5.1 Propriedade genérica . . . . . . . . . . . . . . . . . . . . . . . . 79

5.2 Existência dos pontos sextáticos . . . . . . . . . . . . . . 80

5.3 Caracterização dos pontos sextáticos e parabólicos . . . . . . . . . . . . . 84

5.4 Classificação de curvas planas . . . . . . . . . . . . . . . . 86 
Referências Bibliográficas 



\section{Introdução}

A geometria diferencial de curvas, tem sido estudada desde antes das idéias que levaram ao cálculo, isto é desde tempos anteriores a Newton e Leibniz. Por outro lado a partir dos trabalhos de Whitney e Thom na década do 50, teve inicio a Teoria de Singularidades que está numa linha direta de descendência do cálculo e é a última das muitas tentativas para compreender a riqueza da geometria e beleza de curvas, superfícies e variedades de maior dimensão. O estudo da geometria de curvas e superfícies no espaço Euclidiano envolvendo suas singularidades relaciona estas duas teorias e permite uma visão global dos principais conceitos de cálculo e geometria diferencial de uma forma natural.

Curvas e suas paralelas e envolventes foram estudadas antes de Newton, por exemplo, o matemático e físico holandês C. Huyghens. Ele estava interessado na propagação de ondas, ele pensou uma paralela como a envolvente de círculos centrados numa curva. Ele também estudou cerca de 1660 a involuta de uma curva, desenvolvendo uma sequência de cordas a partir da curva.

A geometria das curvas e superfícies no plano ou no espaço, é estudada por meio de funções de valor real sobre as curvas (como a distância ao quadrado e altura). R Thom nos anos 1960, foi o primeiro em explorar estas ideias mas ele usou funções reais para obter propriedades topológicas.

A extração de informação local sobre as funções a partir dos termos de sua série de Taylor (de seus jatos) tem sido um dos temas centrais do cálculo, desde a epoca de Newton e Leibniz.

Por outro lado, em 1870, já se conheciam varias geometrias: Euclidiana, afim, projetiva, inversiva, geometria esférica e não-euclidiana. Para unificar estes conceitos num único ponto de vista Felix Klein, desenvolveu um programa "Erlangen Program". Sua idéia é bem elegante, considera a geometria como um espaço junto com um grupo de transformações desse espaço, e propriedades de figuras que são invariantes por transformações. 
Felix Klein in 1872, declarou em seu famoso programa, "Erlanger Proogram", geometria é o estudo de invariantes em relação a um determinado grupo de transformação.

Geometria diferencial Euclidiana clássica é o estudo de invariantes diferenciais em relação ao grupo dos movimentos rígidos e geometria diferencial afim é o estudo de invariantes diferenciais em relação ao grupo de transformações afins.

Este trabalho esta baseado no trabalho de Shyuichi Izumiya e Takasi Sano[11], a ideia principal é mostrar que a geometria diferencial afim é mais apropriada para descrever curvas sem pontos de inflexão. Para isto são definidas em [11] duas funções suporte sobre uma curva plana, as chamadas função distância ao cubo afim e função altura afim, e são estudadas as singularidades destas funções para obter informação da geometria da evoluta afim e da curva normal afim associadas a esta curva.

No Capítulo 1, revisamos conceitos de curvas e conceitos relacionados a estas tais como comprimento de arco, curvatura, retas tangentes, retas normais, equações de serret-frenet. Também apresentamos os conceitos de contato entre curvas e das funções distância ao quadrado e altura. As definições e resultados deste capítulo foram seguidos de [3].

No Capítulo 2, revisamos conceitos de teoria de singularidades tais como jatos, equivalência a direita e desdobramentos. Aqui enunciamos os critérios para (p)versalidade e versalidade que serão usados para demonstrar nosso principal resultado.

No Capítulo 3, primeiro apresentamos a geometria Euclidiana e geometria afim do ponto de vista de Klein, depois estudamos o espaço afim para poder apresentar os conceitos próprios de geometria diferencial afim, tais como comprimento de arco afim, curvatura afim, vértice afim, inflexão afim, reta tangente e normal afim, evoluta afim e as funções suporte afim. Aqui se exibem caracterizações da evoluta afim e curvatura afim via as funções distância ao cubo afim e altura afim, respectivamente. Também neste capítulo se prova que a curvatura e o comprimento de arco são invariantes equiafins. As notações e resultados seguem basicamente de [11],[3], [2],[14].

No Capítulo 4, apresentamos a aberração como em [15]. Neste capítulo damos interpretação geométrica de modo natural para o ordem do contato, para isto usamos os conceitos de aberração e de cônica osculante. Além disso, obtemos relações para o raio de aberração, eixo de aberração e fundamentalmente deduzimos relações explícitas que caracterizam os pontos de uma curva que fazem contato de ordem 4, 5 e 6 com a cônica osculante, em função da curvatura e suas derivadas.

No Capítulo 5, definimos as propriedades genéricas como em [3] e também pontos parabólicos e pontos sextáticos. Estudamos a existência de pontos sextáticos e caracterizamos os 
pontos parabólicos e sextáticos via curvatura afim. Por último estudamos a geometria da evoluta afim e normal afim.

Ressaltamos que para facilitar a leitura desta dissertação, optamos por incluir todas as definições e resultados básicos sobre curvas tanto na geometria Euclidiana como na geometria afim. Mesmo tendo tornado a dissertação um pouco mais longa, entendemos que a inclusão destes conceitos em ambas geometrias facilita a comparação entre ambas permitindo um melhor conhecimento de conceitos da geometria afim. 


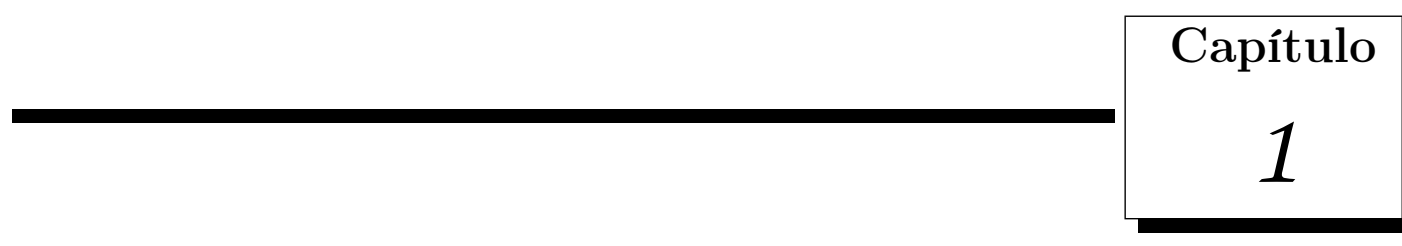

\section{Curvas no espaço Euclidiano}

Neste capítulo, apresentamos as definições básicas sobre curvas espacias e curvas planas no sentido clássico da geometria diferencial de curvas, estudamos as funções definidas em curvas e também a noção de contato entre curvas.

Para fixar a notação estamos considerando o espaço euclideano $\mathbb{R}^{n}$, ou seja o conjunto de n-uplas de números reais,

$$
\left(x_{1}, \ldots, x_{n}\right), x_{i} \in \mathbb{R}, \forall i=1, \ldots, n
$$

com produto escalar usual,

$$
x . y=x_{1} y_{1}+\ldots+x_{n} y_{n},
$$

e com comprimento de $x$ dado por,

$$
\|x\|=(x . x)^{\frac{1}{2}}=\left(x_{1}^{2}+\ldots+x_{n}^{2}\right)^{\frac{1}{2}}
$$

onde $x=\left(x_{1}, \ldots, x_{n}\right), y=\left(y_{1}, \ldots, y_{n}\right)$ em $\mathbb{R}^{n}$.

Neste trabalho entendemos funções suaves como funções de classe $C^{\infty}$.

\subsection{Curva parametrizada regular - comprimento de arco}

Nesta seção estudamos os conceitos de curvas parametrizadas regulares, definimos mudança de parâmetro e em particular revisamos o conceito de uma curva reparametrizada por comprimento de arco. 
Definição 1.1. Uma curva parametrizada no espaço Euclidiano $\mathbb{R}^{n}$ é uma função $\gamma: I \rightarrow \mathbb{R}^{n}$ onde I é um intervalo aberto de $\mathbb{R}$ e $\gamma(t)=\left(\gamma_{1}(t), \ldots, \gamma_{n}(t)\right)$ com $\gamma_{i}$ de classe $C^{\infty}, \forall i=1, \ldots, n$. A curva $\gamma$ é chamada regular se $\gamma^{\prime}(t) \neq 0, \forall t \in I$.

Seja $\gamma: I \rightarrow \mathbb{R}^{n}$ uma curva regular. O vetor $v=\gamma(t+h)-\gamma(t)$ corresponde ao segmento da corda desde $\gamma(t)$ para $\gamma(t+h)$.

Observemos que o vetor,

$$
\left(\gamma_{1}^{\prime}(t), \ldots, \gamma_{n}^{\prime}(t)\right)=\gamma^{\prime}(t)=\lim _{h \rightarrow 0} \frac{\gamma(t+h)-\gamma(t)}{h}
$$

tem por direção o limite destas cordas, a dizer a tangente em $\gamma(t)$.

Definição 1.2. Seja $\gamma: I \rightarrow \mathbb{R}^{n}$ uma curva regular, o vetor $T(t)=\frac{\gamma^{\prime}(t)}{\left\|\gamma^{\prime}(t)\right\|}$ é chamado vetor tangente unitário a $\gamma$ em $t$. O comprimento $\left\|\gamma^{\prime}(t)\right\|$ é chamada velocidade de $\gamma$ em $t$. Dizemos que $\gamma$ tem velocidade unitária se $\left\|\gamma^{\prime}(t)\right\|=1$ para todo $t \in I$. A reta tangente a $\gamma$ em $t$ é a reta através de $\gamma(t)$ na direção $T(t)$.

Para $n=2$ a equação da reta tangente a $\gamma: I \rightarrow \mathbb{R}^{2} \operatorname{com} \gamma(t)=\left(\gamma_{1}(t), \gamma_{2}(t)\right)$ em $t$ é dada por

$$
\left(x_{1}-\gamma_{1}(t)\right) \gamma_{2}^{\prime}(t)-\left(x_{2}-\gamma_{2}(t)\right) \gamma_{1}^{\prime}(t)=0 \text {. }
$$

Observação 1.3. Ressaltemos os seguintes fatos que serão usados posteriormente,

1. se $x, y: I \rightarrow \mathbb{R}^{n}$ são curvas suaves então a função $x . y: I \rightarrow \mathbb{R}^{2}$ definida por $(x . y)(t)=x(t) . y(t), \forall t \in I$, define uma curva suave, além disso tem-se a seguinte relação

$$
(x . y)^{\prime}=x \cdot y^{\prime}+x^{\prime} \cdot y
$$

2. se $x: I \rightarrow \mathbb{R}^{n}$ é uma curva suave tal que $x . x=1$ (isto é $x(t) . x(t)=1, \forall t \in I$ ), então $x \cdot x^{\prime}=0$.

A seguir introduzimos a noção de mudança de parâmetros.

Definição 1.4. Seja $\gamma: I \rightarrow \mathbb{R}^{n}$ uma curva regular. Uma mudança de parâmetro para $\gamma$ é uma função $h: J \rightarrow I$, onde $J$ é um intervalo aberto, satisfazendo:

1. h é suave,

2. $h^{\prime}(s) \neq 0, \forall s \in J$,

3. $h(J)=I$. 
A curva $\delta: J \rightarrow \mathbb{R}^{n}$ dada por $\delta(s)=\gamma(h(s))$ é dita como sendo obtida de $\gamma$ por mudança de parâmetro $h$ ou que $\delta$ é uma reparametrização de $\gamma$.

Notemos os seguintes fatos,

- a curva $\delta$ é regular, desde que $\gamma$ seja regular,

- $h: J \rightarrow I$ é uma bijeção de $J$ em $I$, logo tem inversa $h^{-1}$ além disso, $h^{-1}$ é suave, desde que $h^{\prime}(s) \neq 0, \forall s \in J$. Assim $h$ é um difeomorfismo de $J$ em $I$.

Proposição 1.5. Seja $g: I \rightarrow \mathbb{R}$ uma função suave e $h: J \rightarrow I$ um difeomorfismo, seja $t_{0} \in I$ e $s_{0}=h^{-1}\left(t_{0}\right)$ então as derivadas $g^{(i)}\left(t_{0}\right), i=1,2, \ldots, k$ são todas zero se, $e$ somente se, as derivadas $(g \circ h)^{(i)}\left(t_{0}\right), i=1,2, \ldots, k$ são todas zero.

Demonstração: Ver ([3], p. 26).

Definição 1.6. O comprimento de arco de uma curva regular $\gamma: I \rightarrow \mathbb{R}^{n}$, medido desde $\gamma\left(t_{0}\right)$, onde $t_{0} \in I$ é,

$$
l(t)=\int_{t_{0}}^{t}\left\|\gamma^{\prime}(u)\right\| d u .
$$

Em particular, se $\gamma$ tem velocidade unitária, então $l(t)=t-t_{0}$.

Observemos que fixando $t_{0} \in I$, o comprimento de arco de $\gamma$ define um difeomorfismo. Com efeito, definindo $l(t)$ como em (1.1), $l$ é suave e $l^{\prime}(t)=\left\|\gamma^{\prime}(t)\right\|>0, \forall t \in I$. Assim $l$ define um difeomorfismo.

Tendo em mente esta observação, usualmente fixamos $t_{0} \in I$ e dizemos que $l$ é uma função comprimento de arco.

Se $\gamma: I \rightarrow \mathbb{R}^{n}$ uma curva regular então $\alpha=\gamma \circ l^{-1}: J \rightarrow \mathbb{R}^{n}$ tem velocidade unitária, neste caso ela é chamada de curva parametrizada por comprimento de arco.

Para demonstrar esta afirmação, seja $\gamma: I \rightarrow \mathbb{R}^{n}$ uma curva regular. Escolhemos $t_{0} \in I$ e definamos $l(t)$ como em (1.1). Como $l$ é um difeomorfismo de $I=(a, b)$ para um intervalo aberto $J=(l(a), l(b))$, consideremos $h=l^{-1}$. Afirmamos que $\alpha=\gamma \circ l^{-1}: J \rightarrow \mathbb{R}^{n}$ tem velocidade unitária.

De fato, como $l(h(s))=s, \forall s \in J$, pela regra da cadeia $l^{\prime}(h(s)) h^{\prime}(s)=1, \forall s \in J$. Por outro lado $\alpha^{\prime}(s)=\gamma^{\prime}(h(s)) h^{\prime}(s)$, daí

$$
\left\|\alpha^{\prime}(s)\right\|=\left\|\gamma^{\prime}(h(s)) h^{\prime}(s)\right\|=\left\|\gamma^{\prime}(h(s)) h^{\prime}(s)\right\| \frac{\left|l^{\prime}(h(s))\right|}{\left|l^{\prime}(h(s))\right|}
$$




$$
=\frac{1}{l^{\prime}(h(s))}\left\|\gamma^{\prime}(h(s))\right\|=1
$$

\subsection{Curvatura e equações de Serret-Frenet}

Outros conceitos associados às curvas são a curvatura e as equações de Serret-Frenet.

Definição 1.7. Seja $\alpha: I \rightarrow \mathbb{R}^{n}$ uma curva regular parametrizada por comprimento de arco onde $\alpha(s)=\left(x_{1}(s), \ldots, x_{n}(s)\right)$, e seja $n \geq 3$, a curvatura de $\alpha$ em s é definida como

$$
k(s)=\left\|T^{\prime}(s)\right\|=\left(\left(x_{1}^{\prime \prime}(s)\right)^{2}+\ldots+\left(x_{n}^{\prime \prime}(s)\right)^{2}\right)^{1 / 2} .
$$

Tem-se $k(s) \geq 0$ e $k$ é uma função suave. No caso que $k(s) \neq 0$, dizemos que o vetor unitário $N(s)$ definido por

$$
N(s)=\frac{T^{\prime}(s)}{k(s)}
$$

é a normal principal. Desde que $\|T(s)\|=1$ então $N(s) \perp T(s)$. No caso que $k(s)=0$, $N(s)$ não é definido.

Definição 1.8. Seja $\gamma: I \rightarrow \mathbb{R}^{n}$ uma curva regular, com função comprimento de arco $l$ (relativo para algum $t_{0} \in I$ ), definimos a curvatura de $\gamma$ em $t, k(t)$ como a curvatura da curva $\alpha=\gamma \circ l^{-1}: J \rightarrow \mathbb{R}^{n}$ em $s=l(t)$.

A curvatura de $\gamma$ em $t$ está dada pela relação,

$$
k(t)=\frac{\left\|T^{\prime}(t)\right\|}{\left\|\gamma^{\prime}(t)\right\|} .
$$

De fato, escrevemos $T(t)$ o vetor tangente unitário de $\gamma$ em $t$ e $T_{\alpha}(s)$ para o vetor unitário a $\alpha$ em $s$; então $T(t)=T_{\alpha}(l(t))$ e daí $T^{\prime}(t)=T_{\alpha}^{\prime}(l(t)) l^{\prime}(t)$. Portanto,

$\left\|T^{\prime}(t)\right\|=\left\|T_{\alpha}^{\prime}(l(t)) l^{\prime}(t)\right\|=k_{\alpha}(l(t)) l^{\prime}(t)=k(t) l^{\prime}(t)$.

\subsubsection{Curvatura e normal para curvas planas}

Seja $\alpha: I \rightarrow \mathbb{R}^{2}$ uma curva com velocidade unitária, com $\alpha(s)=(x(s), y(s)), \forall s \in I$. Como $\alpha$ tem velocidade unitária então $\left\|\alpha^{\prime}\right\|=1 \mathrm{e}$, portanto, $\alpha^{\prime} \cdot \alpha^{\prime \prime}=0$. Agora definimos a normal unitária $N(s)$ como sendo obtida desde $T(s)=\alpha^{\prime}(s)$ pela rotação no sentido anti-horário um ângulo $\frac{1}{2} \pi$. Assim $T(s)=\left(x^{\prime}(s), y^{\prime}(s)\right)$ e $N(s)=\left(-y^{\prime}(s), x^{\prime}(s)\right)$. Por outro lado, $T^{\prime}(s)=\alpha^{\prime \prime}(s)$ é perpendicular a $T(s)=\alpha^{\prime}(s)$. Desde que $T(s)$ e $T^{\prime}(s) \in \mathbb{R}^{2}$, existe $k(s) \in \mathbb{R}$ tal que

$$
T^{\prime}(s)=k(s) N(s) .
$$


Chamamos $k(s)$ a curvatura de $\alpha$ em $s$. Note que $|k(s)|=\left\|T^{\prime}(s)\right\|$.

Com $\alpha$ como acima tem-se

$$
T^{\prime}=\alpha^{\prime \prime}=\left(x^{\prime \prime}, y^{\prime \prime}\right)=k N \text {. }
$$

Pela equação (1.4), $k=T^{\prime} \cdot N=\left(x^{\prime \prime}, y^{\prime \prime}\right) \cdot\left(-y^{\prime}, x^{\prime}\right)$, então

$$
k=x^{\prime} y^{\prime \prime}-x^{\prime \prime} y^{\prime}
$$

Em particular $k$ é uma função suave de $s, \forall s \in I$.

Seja $\alpha: I \rightarrow \mathbb{R}^{2}$ curva com velocidade unitária e suponhamos que o ângulo entre a tangente de $\alpha$ em $s$ e o eixo- $x$ é $\psi(s)$ com $0 \leq \psi(s)<2 \pi$, assim temos definida uma função suave $\psi: I \rightarrow \mathbb{R}$, tal que

$$
\cos \psi(s)=x^{\prime}(s), \quad \operatorname{sen} \psi(s)=y^{\prime}(s)
$$

Tem-se $x^{\prime \prime}(s)=-\psi^{\prime}(s) \operatorname{sen} \psi(\mathrm{s}), \quad y^{\prime \prime}(s)=\psi^{\prime}(s) \cos \psi(s)$ portanto

$$
k(s)=\psi^{\prime}(s)=\frac{d \psi}{d s} .
$$

Consideremos agora o caso de uma curva arbitrária $\gamma: I \rightarrow \mathbb{R}^{2}, \gamma(t)=(x(t), y(t))$. Lembremos que $k(t)$ é definida como a curvatura da curva de velocidade unitária $\alpha=\gamma \circ l^{-1}$ em $l(t)$. Por outro lado temos

$$
\begin{aligned}
& T(t)=\frac{\left(x^{\prime}(t), y^{\prime}(t)\right)}{\left(\left(x^{\prime}(t)\right)^{2}+\left(y^{\prime}(t)\right)^{2}\right)^{\frac{1}{2}}}, \\
& N(t)=\frac{\left(-y^{\prime}(t), x^{\prime}(t)\right)}{\left(\left(x^{\prime}(t)\right)^{2}+\left(y^{\prime}(t)\right)^{2}\right)^{\frac{1}{2}}},
\end{aligned}
$$

e que $l^{\prime}(t)=\left(\left(x^{\prime}(t)\right)^{2}+\left(y^{\prime}(t)\right)^{2}\right)^{\frac{1}{2}}$ e que $\alpha$. Assim

$$
\gamma^{\prime}(t)=\alpha^{\prime}(l(t)) l^{\prime}(t)=T(l(t)) l^{\prime}(t) \text { e } \gamma^{\prime \prime}(t)=\alpha^{\prime \prime}(l(t))\left(l^{\prime}(t)\right)^{2}+\alpha^{\prime}(l(t)) l^{\prime \prime}(t) .
$$

Desde que $\alpha$ tem velocidade unitária obtem-se $\alpha^{\prime \prime}(l(t))=k_{\alpha}(l(t)) N_{\alpha}(l(t))$ e $T_{\alpha}(l(t))=\alpha^{\prime}(l(t))$ é o vetor tangente unitário, daí $\gamma^{\prime \prime}(t) \cdot N_{\alpha}(l(t))=\left(\alpha^{\prime \prime}(l(t))\left(l^{\prime}(t)\right)^{2}+\alpha^{\prime}(l(t)) l^{\prime \prime}(t)\right) \cdot N_{\alpha}(l(t))=k_{\alpha}(l(t))\left(l^{\prime}(t)\right)^{2}$.

Segue-se,

$$
k=\frac{x^{\prime} y^{\prime \prime}-x^{\prime \prime} y^{\prime}}{\left(\left(x^{\prime}\right)^{2}+\left(y^{\prime}\right)^{2}\right)^{\frac{3}{2}}} .
$$




\subsubsection{Fórmulas de Serret-Frenet para curvas planas}

Da equação (1.3) temos $T^{\prime}(s)=k(s) N(s)$. Por outro lado $\|N(s)\|=1$. Assim $N(s) \cdot N^{\prime}(s)=1$ e então existe $\lambda(s) \in \mathbb{R}$ tal que $N^{\prime}(s)=\lambda(s) T(s)$. Também derivando $T(s) \cdot N(s)=0$ segue $T^{\prime}(s) N(s)+T(s) \cdot N^{\prime}(s)=0$ e, substituindo as relações acima nesta equação, tem-se

$$
k(s) N(s) \cdot N(s)+\lambda(s) T(s) \cdot T(s)=0 .
$$

Daí $k(s)+\lambda(s)=0$ e assim obtemos a seguinte equação

$$
N^{\prime}=-k T
$$

As fórmulas (1.4) e(1.7) são as fórmulas de Serret-Frenet para curvas planas.

\subsubsection{Fórmulas de Serret-Frenet para curvas no espaço}

Seja $\alpha: I \rightarrow \mathbb{R}^{3}$ curva com velocidade unitária e $\alpha^{\prime}(s)=T(s)$ o vetor tangente unitário, também a curvatura é dada por $k(s)=\left\|T^{\prime}(s)\right\|$ e, para $k(s) \neq 0$, existe uma normal principal $N(s)$ perpendicular para $T(s)$. Da equação (1.2) temos $T^{\prime}(s)=k(s) N(s)$. No que segue consideramos curvas $\operatorname{com} k(s) \neq 0$.

Como $T(s)$ e $N(s)$ são vetores unitários e perpendiculares, consideremos o único vetor $B(s)$ chamado vetor binormal perpendicular a ambos e tal que, quando $T(s), N(s)$, $B(s)$ são escritos como vetores coluna nessa ordem, estes constituem uma matriz $3 \times 3$ com determinante 1 .

Definição 1.9. O plano passando por $\alpha(s)$ e gerado por $N(s)$ e $B(s)$ é chamado o plano normal em s e o plano passando por $\alpha(s)$ e gerado por $T(s)$ e $N(s)$ é chamado o plano osculador.

Agora para efeitos de cálculo vamos omitir $s$. Como $N$ é unitário, então $N^{\prime}$ é perpendicular com $N$, assim existem $\lambda, \tau \in \mathbb{R}$ tal que $N^{\prime}=\lambda T+\tau B(\lambda, \tau$ dependem de $s)$. Por outro lado, como $T . N=0$, derivando temos $T^{\prime} \cdot N+T \cdot N^{\prime}=0$. Logo substituindo $T^{\prime}$ e $N^{\prime}$ nesta equação, tem-se

$$
k N \cdot N+T \cdot(\lambda T+\tau B)=0
$$

$\operatorname{assim} \lambda=-k$. Portanto

$$
N^{\prime}(s)=-k(s) T(s)+\tau(s) B(s)
$$

para algum $\tau(s) \in \mathbb{R}$. Este número $\tau(s)$ é chamado torção da curva em $s$. Logo, como 
$k(s) \neq 0, \tau$ é uma função suave de $s$.

Além disso, como $B^{\prime}$ é perpendicular com $B$, existem $\mu, \nu \in \mathbb{R}$ tal que $B^{\prime}=\mu T+\nu N$. Usando $B . T=0$ temos $B^{\prime} . T+B \cdot T^{\prime}=0$ e assim $\mu+0=0$. Utilizando $B . N=0$ obtemos de forma similar $\nu+\tau=0, \log 0 B^{\prime}(s)=-\tau(s) N(s)$.

Portanto

$$
\left\{\begin{array}{c}
T^{\prime}(s)=k(s) N(s) \\
N^{\prime}(s)=-k(s) T(s)+\tau(s) B(s), \\
B^{\prime}(s)=-\tau(s) N(s)
\end{array}\right.
$$

que são as equações de Serret-Frenet para curvas no espaço.

\subsection{Contato entre curvas}

Para $F: \mathbb{R}^{n} \rightarrow \mathbb{R}$ uma função suave e $\gamma: I \rightarrow \mathbb{R}^{n}$ uma curva regular definimos o contato da curva $\gamma \operatorname{com} F^{-1}(0)$.

Definição 1.10. Dizemos que uma curva $\gamma$ e $F^{-1}(0)$ tem $\boldsymbol{k}$-ponto de contato para $t=t_{0}$ (mais precisamente em $p=\gamma\left(t_{0}\right)$ ) se a função $g: I \rightarrow \mathbb{R}$ definida por

$$
g(t)=F(\gamma(t))=F\left(\gamma_{1}(t), \ldots, \gamma_{n}(t)\right)
$$

satisfaz

$$
g\left(t_{0}\right)=g^{\prime}\left(t_{0}\right)=\ldots=g^{(k-1)}\left(t_{0}\right)=0 \text { e } g^{(k)}\left(t_{0}\right) \neq 0 .
$$

Neste caso dizemos que a ordem do contato é $k$.

Além disso, se só sabemos que

$$
g\left(t_{0}\right)=g^{\prime}\left(t_{0}\right)=\ldots=g^{(k-1)}\left(t_{0}\right)=0
$$

dizemos que curva $\gamma$ e $F^{-1}(0)$ tem ao menos $k$-ponto de contato para $t=t_{0}$ ou que $o$ ordem do contato é $\geq k$.

Exemplo 1.11. Seja $\gamma: I \rightarrow \mathbb{R}^{n}$ curva regular. Fixando $t_{0} \in I$ consideremos a função

$$
F: \mathbb{R}^{n} \rightarrow \mathbb{R}, \text { definida por } F(x)=\left(x-\gamma\left(t_{0}\right)\right) \cdot u,
$$

onde $u$ é um vetor fixo em $\mathbb{R}^{n}$, com $F^{-1}(0)=\left\{x \in \mathbb{R}^{n} ;\left(x-\gamma\left(t_{0}\right)\right) \cdot u=0\right\}$. Agora consideremos a função

$$
g: I \rightarrow \mathbb{R}, \text { definida por } g(t)=F(\gamma(t))=\left(\gamma(t)-\gamma\left(t_{0}\right)\right) . u
$$


Como $g\left(t_{0}\right)=0$ então $\gamma$ e $F^{-1}(0)$ tem ao menos 1-ponto de contato em $t=t_{0}$.

Derivando g, tem-se $g^{\prime}(t)=\gamma^{\prime}(t) . u, \operatorname{logo} \gamma$ e $F^{-1}(0)$ tem ao menos 2-ponto de contato em $t=t_{0}$ se, e somente se, $u$ é ortogonal a $\gamma^{\prime}\left(t_{0}\right)$.

Exemplo 1.12. Seja $F: \mathbb{R}^{2} \rightarrow \mathbb{R}$ a segunda projeção $F\left(x_{1}, x_{2}\right)=x_{2} \operatorname{com} F^{-1}(0)=$ $\mathbb{R} \times\{0\}$, seja $\gamma: \mathbb{R} \rightarrow \mathbb{R}^{2}$ definida por $\gamma(t)=\left(t, t^{k}\right)$, estudamos o contato de $\gamma$ e $F^{-1}(0)$ no ponto $t=0$. Consideramos $g: \mathbb{R} \rightarrow \mathbb{R}$ definida por $g(t)=F(\gamma(t))$, assim $g(t)=t^{k}$ derivando têm-se

$$
g^{\prime}(t)=k t^{k-1}, g^{\prime \prime}(t)=(k)(k-1) t^{k-2}, \ldots, g^{(k-1)}(t)=k ! t \text { e } g^{(k)}(t)=k !
$$

para $t=0, g(0)=g^{(1)}(0)=\ldots=g^{(k-1)}(0)=0$ e $g^{(k)(0)}=k ! \neq 0$ portanto $\gamma$ e $F^{-1}(0)$ tem k-ponto de contato em $t=0$.

Proposição 1.13. Sejam $F: \mathbb{R}^{n} \rightarrow \mathbb{R}$ função suave, $\gamma: I \rightarrow \mathbb{R}^{n}$ uma curva regular $e$ $L: \mathbb{R}^{n} \rightarrow \mathbb{R}^{n}$ uma aplicação linear invertivel ou uma translação.

Se $G=F \circ L: \mathbb{R}^{n} \rightarrow \mathbb{R}$ e $\delta=L^{-1} \circ \gamma$ tais que $F \circ \gamma$ é suave então a ordem de contato de $G^{-1}(0)$ e $\delta$ para $t=t_{0}$ é igual igual ao ordem de contato de $F^{-1}(0)$ e $\gamma$ para $t=t_{0}$.

Demonstração: Basta ver que $(G \circ \delta)=(F \circ L) \circ\left(L^{-1} \circ \gamma\right)=(F \circ \gamma)$.

A proposição acima diz que o ordem do contato é invariante por aplicações lineares inversíveis e por translações, em particular o contato é invariante por rotações e reflexões.

Lema 1.14. Sejam $\gamma: I \rightarrow \mathbb{R}^{n}$ uma curva regular e $F, F_{1}: \mathbb{R}^{n} \rightarrow \mathbb{R}$ funções tais que $F \circ \gamma, F_{1} \circ \gamma$ são suaves. Seja $t_{0} \in I, p=\gamma\left(t_{0}\right)$ e suponhamos que $F_{1}(p) \neq 0$. Então a ordem do contato de $\gamma$ com $\left(F F_{1}\right)^{-1}(0)$ em $t_{0}$ é $k$ se, e somente se, o ordem do contato de $\gamma \operatorname{com~} F^{-1}(0)$ em $t_{0}$ é $k$.

Demonstração: Consideremos $g(t)=F(\gamma(t))$ e $h(t)=F F_{1}(\gamma(t))=F\left(\gamma(t) F_{1}(\gamma(t))\right.$ derivando $h$ (usando a regra de leibniz) obtemos as seguintes relações:

$h\left(t_{0}\right)=g\left(t_{0}\right) F_{1}(p)$

$h^{\prime}\left(t_{0}\right)=g^{\prime}\left(t_{0}\right) F_{1}(p)+g\left(t_{0}\right) F_{1}^{\prime}(p) \gamma^{\prime}\left(t_{0}\right)$

$h^{\prime \prime}\left(t_{0}\right)=g^{\prime \prime}\left(t_{0}\right) F_{1}(p)+2 g^{\prime}\left(t_{0}\right) F_{1}^{\prime}(p) \gamma^{\prime}\left(t_{0}\right)+g\left(t_{0}\right) F_{1}^{\prime}(p)\left(\gamma^{\prime}\left(t_{0}\right)^{2}+\gamma^{\prime \prime}\left(t_{0}\right)\right)$

assim

$$
h^{(n)}\left(t_{0}\right)=g^{(n)}\left(t_{0}\right) F_{1}(p)+\text { termos envolvendo derivadas de } g, \gamma \text { e } F_{1},
$$

Como $F_{1}(p) \neq 0, h\left(t_{0}\right)=\ldots=h^{(k-1)}\left(t_{0}\right)=0$ e $h^{(k)}\left(t_{0}\right) \neq 0$ se, e somente se, $g\left(t_{0}\right)=\ldots=g^{(k-1)}\left(t_{0}\right)=0$ e $g^{(k)}\left(t_{0}\right) \neq 0$. 
Proposição 1.15. Seja $\gamma: I \rightarrow \mathbb{R}^{2}$ uma curva regular, $l$ uma função comprimento de arco e $\alpha=\gamma \circ l^{-1}$. Então $k^{\prime}\left(t_{0}\right)=\ldots=k^{(n)}\left(t_{0}\right)=0$ se, e somente se, $k_{\alpha}^{\prime}\left(s_{0}\right)=\ldots=$ $k_{\alpha}^{(n)}\left(s_{0}\right)=0$, onde $s_{0}=l\left(t_{0}\right)$.

Demonstração: Por definição de curvatura tem-se $k(t)=k_{\alpha}(l(t))$, derivando e considerando $t=t_{0}$ temos

$k^{\prime}\left(t_{0}\right)=k_{\alpha}\left(s_{0}\right) l^{\prime}\left(t_{0}\right)$,

$k^{\prime \prime}\left(t_{0}\right)=k_{\alpha}^{\prime \prime}\left(s_{0}\right) l^{\prime}\left(t_{0}\right)^{2}+k_{\alpha}^{\prime}\left(s_{0}\right) l^{\prime \prime}\left(t_{0}\right)$,

$k^{\prime \prime \prime}\left(t_{0}\right)=k_{\alpha}^{\prime \prime \prime}\left(s_{0}\right) l^{\prime}\left(t_{0}\right)^{3}+3 k_{\alpha}^{\prime \prime}\left(s_{0}\right) l^{\prime}\left(t_{0}\right) l^{\prime \prime}\left(t_{0}\right)+k_{\alpha}^{\prime}\left(s_{0}\right) l^{\prime \prime \prime}\left(t_{0}\right)$,

portanto, para $n \in \mathbb{N}$, obtemos

$$
k^{(n)}\left(t_{0}\right)=k_{\alpha}^{(n)}\left(s_{0}\right) l^{\prime}\left(t_{0}\right)^{n}+\text { termos envolvendo derivadas de } k \text { e } l
$$

Como $l^{\prime}\left(t_{0}\right)=\left\|\gamma^{\prime}\left(t_{0}\right)\right\| \neq 0$, segue-se a proposição.

Proposição 1.16. Seja $\gamma: I \rightarrow \mathbb{R}^{2}$ uma curva parametrizada da forma $\gamma(t)=(t, y(t))$, onde $y: I \rightarrow \mathbb{R}$ é uma função suave que satisfaz $y(0)=y^{\prime}(0)=0$. Então $\gamma$ tem ao menos n-ponto de contato com o eixo-x no ponto $t=0$ ( $n \geq 3)$ se, e somente se, $k(0)=\ldots=k^{(n-3)}(0)=0$.

Demonstração: Consideremos a segunda projeção $F(x, y)=y \operatorname{com} F^{-1}(0)$ o eixo- $x$, tem-se $F(\gamma(t))=y(t)$, assim para saber a ordem do contato de $\gamma$ e o eixo- $x$, olhamos as derivadas de $y$.

Por outro lado, da equação $(1.6), k(t)=\frac{y^{\prime \prime}(t)}{\left(1+\left(y^{\prime}(t)\right)^{2}\right)^{\frac{3}{2}}}$ daí,

$$
y^{\prime \prime}(t)=k(t)\left(1+\left(y^{\prime}(t)\right)^{2}\right)^{\frac{3}{2}},
$$

por derivação obtem-se,

$y^{\prime \prime \prime}(t)=k^{\prime}(t)\left(1+\left(y^{\prime}(t)\right)^{2}\right)^{\frac{3}{2}}+3 k(t) y^{\prime}(t) y^{\prime \prime}(t)\left(1+\left(y^{\prime}(t)\right)^{2}\right)^{\frac{1}{2}}$.

Em geral para $p \in \mathbb{N}$

$$
y^{(p)}(t)=k^{(p-2)}(t)\left(1+\left(y^{\prime}(t)\right)^{2}\right)^{\frac{3}{2}}+\text { termos envolvendo derivadas e } y \text { e } k .
$$

Notemos que os termos envolvendo as derivadas de $y$ tem ordem $<p$ e os termos envolvendo as derivadas de $k$ tem ordem $<p-2$, logo segue o resultado.

Corolário 1.17. Seja $\gamma: I \rightarrow \mathbb{R}^{2}$ uma curva regular, $\gamma$ tem ao menos $n$-ponto de contato com sua reta tangente em $\gamma\left(t_{0}\right)$ se, e somente se, $k\left(t_{0}\right)=\ldots=k^{(n-3)}\left(t_{0}\right)=0$. 
Demonstração: Por rotação, translação e pela Proposição (1.13) podemos considerar, $\gamma(t)=(t, y(t))$, com $\gamma(0)=(0,0)$ e $\gamma^{\prime}(0)=(1,0)$. Agora pela proposição acima $\gamma$ tem ao menos $n$-ponto de contato em $t=0$ se, e somente se, $k(0)=\ldots=k^{(n-3)}(0)$.

Definição 1.18. Seja $\gamma: I \rightarrow \mathbb{R}^{2}$ uma curva plana regular.

1. Um ponto $p=\gamma\left(t_{0}\right)$ é um vértice ordinário da curva plana $\gamma$ se existe um círculo $C$ tal que a curva $\gamma$ e $C$ tem 4-ponto de contato para $t=t_{0}$.

2. Um ponto $p=\gamma\left(t_{0}\right)$ é um vértice degenerado da curva plana $\gamma$ se existe um círculo $C$ tal que $\gamma$ e $C$ tem ao menos 5-ponto de contato para $t=t_{0}$.

Na Definição (1.18) para encontrar os vértices de uma curva $\gamma$, definimos a função $F: \mathbb{R}^{2} \rightarrow \mathbb{R}$ dada por $F(x, y)=(x-a)^{2}+(y-b)^{2}-R^{2}$, logo consideramos a função $g: I \rightarrow \mathbb{R}$ dada por $g(t)=F(\gamma(t))$ e devemos encontrar valores $a, b$ e $R$ tais que $\gamma$ e $F^{-1}(0)$ tenham 4-ponto de contato para algum $t=t_{0}$ da curva $\gamma$.

Exemplo 1.19. A elipse definida pela equação $x^{2}+4 y^{2}=4$ tem 4 vértices ordinarios.

De fato, parametrizando a elipse por $\gamma(t)=(2 \cos t$, sent $)$, seja $p_{0}=\left(2 \cos t_{0}\right.$, sent $\left.t_{0}\right)$ um ponto da elipse, agora consideremos $g(t)=(2 \cos t-a)^{2}+(\operatorname{sen} \mathrm{t}-\mathrm{b})^{2}-\mathrm{R}^{2}$ e procuremos valores para $a, b$ e $R$ tais que $p_{0}$ seja um vértice.

- $g\left(t_{0}\right)=0$ se, e somente se, $\left(2 \cos t_{0}-a\right)^{2}+\left(\operatorname{sen} t_{0}-\mathrm{b}\right)^{2}=\mathrm{R}^{2}$.

- $g^{\prime}(t)=-2 \operatorname{sen} t(2 \cos \mathrm{t}-\mathrm{a})+2 \cos \mathrm{t}(\operatorname{sen} \mathrm{t}-\mathrm{b})$ então $g^{\prime}\left(t_{0}\right)=0$ se, e somente se,

$$
0=\left((a, b)-\left(2 \cos t_{0}, \operatorname{sen} t_{0}\right)\right) \cdot\left(-2 \operatorname{sen} t_{0}, \cos t_{0}\right),
$$

isto é quando, $(a, b)$ pertence à normal da elipse em $\gamma\left(t_{0}\right)$.

- $g^{\prime \prime}(t)=-3 \cos 2 t+2 a \cos t+b \operatorname{sen} t$ então $g^{\prime \prime}\left(t_{0}\right)=0$ se, e somente se,

$$
a\left(2 \cos t_{0}\right)+b\left(\operatorname{sen} t_{0}\right)=3 \cos 2 t_{0}
$$

também $g^{\prime}\left(t_{0}\right)=0$ se e somente se

$$
a\left(2 \operatorname{sen} t_{0}\right)+b\left(-\cos t_{0}\right)=3 \operatorname{sen} t_{0} \cos t_{0} .
$$

Assim, desenvolvendo o sistema de equações tem-se $(a, b)=\left(\frac{3}{2} \cos ^{3} t_{0},-3 \operatorname{sen}^{3} t_{0}\right)$. 
- $g^{\prime \prime \prime}(t)=6 \operatorname{sen} 2 \mathrm{t}-2 \mathrm{a} \operatorname{sen} \mathrm{t}+\mathrm{b} \cos \mathrm{t}, \log \mathrm{O}$

$$
g\left(t_{0}\right)=g^{\prime}\left(t_{0}\right)=g^{\prime \prime}\left(t_{0}\right)=g^{\prime \prime \prime}\left(t_{0}\right)=0
$$

se, e somente se, sent $t_{0} \cos t_{0}=0$, de onde sent $t_{0}=0$ ou cost $t_{0}=0$ e portanto $t_{0}=0, \pi$ ou $t_{0}=\frac{\pi}{2}, \frac{3 \pi}{2}$.

- $g^{(i v)}\left(t_{0}\right)=9 \cos 2 t_{0} \neq 0$ em $t_{0}=0, \pi, \frac{\pi}{2}, \frac{3 \pi}{2}$, segue-se que a elipse tem 4 vértices.

Mais geralmente considerando a elipse $\frac{x^{2}}{A^{2}}+\frac{y^{2}}{B^{2}}=1$ parametrizada por $(A \cos t, B \operatorname{sen} \mathrm{t})$, verifica que, para qualquer $t$, existe exatamente um ponto $(a, b)$ tal que o círculo com centro $(a, b)$ pasando por $(A \cos t, B$ sent) tem ao menos 3 -ponto de contato, os centros destes círculos são dados por

$$
(a, b)=\left(\frac{\left(A^{2}-B^{2}\right) \cos ^{3} t}{A}, \frac{\left(B^{2}-A^{2}\right) \operatorname{sen}^{3} \mathrm{t}}{B}\right)
$$

Estes círculos são chamados círculos osculadores da elipse.

Definição 1.20. Seja $\gamma: I \rightarrow \mathbb{R}^{2}$ uma curva plana regular.

1. Um ponto $p=\gamma\left(t_{0}\right)$ é uma inflexão ordinaria da curva plana $\gamma$ se a reta tangente $L$ em $t=t_{0}$ é tal que a curva $\gamma$ e L tem 3-ponto de contato para $t=t_{0}$.

2. Um ponto $p=\gamma\left(t_{0}\right)$ é uma inflexão degenerada da curva plana $\gamma$ se a reta tangente $L$ em $t=t_{0}$ é tal que a curva $\gamma$ e $L$ tem ao menos 4-ponto de contato para $t=t_{0}$.

\subsection{Funções definidas sobre curvas}

Nesta seção estudamos duas funções de muita utilidade na Teoria de Singularidades, estas são a Função Altura e a Função Distância ao Quadrado, as quais são definidas sobre curvas.

Seja $\gamma: I \rightarrow \mathbb{R}^{n}$ uma curva regular e $F: \mathbb{R}^{n} \rightarrow \mathbb{R}$ uma função, então $F$ se considera como definida na curva, quando tem sentido a função composta $F \circ \gamma: I \rightarrow \mathbb{R}$.

Definição 1.21. Seja $u \in \mathbb{R}^{n}$. A função distância ao quadrado sobre $\gamma$ desde $u$ é a função $f_{d}: I \rightarrow \mathbb{R}$ definida por

$$
f_{d}(t)=\|\gamma(t)-u\|^{2}=(\gamma(t)-u) \cdot(\gamma(t)-u) .
$$


Definição 1.22. Seja $u \in \mathbb{R}^{n}$ um vetor unitário fixado. A função altura sobre $\gamma$ na direção de u é a função $f_{h}: I \rightarrow \mathbb{R}$ definida por

$$
f_{h}(t)=\gamma(t) \cdot u
$$

A seguir apresentaremos o teorema que relaciona o contato de curvas com as funções distância ao quadrado e função altura.

Teorema 1.23. Seja $\gamma: I \rightarrow \mathbb{R}^{2}$ uma curva regular

1. A curva $\gamma$ e o círculo $C$ centrado em u e passando por $\gamma\left(t_{0}\right)$ tem $k$-ponto de contato, para $t=t_{0}$ se, e somente se, a função distância ao quadrado $f_{d}$ sobre $\gamma$ desde $u$ satisfaz $f_{d}^{(i)}\left(t_{0}\right)=0, i=1, \ldots, k-1 ; f_{d}^{(k)}\left(t_{0}\right) \neq 0$.

2. A curva $\gamma$ e a reta tangente $L$ passando por $\gamma\left(t_{0}\right)$ tem $k$-ponto de contato, para $t=t_{0}$ se, e somente se, a função altura $f_{h}$ sobre $\gamma$ na direção de u perpendicular a $T\left(t_{0}\right)$ satisfaz $f_{h}^{(i)}\left(t_{0}\right)=0, i=1, \ldots, k-1 ; f_{h}^{(k)}\left(t_{0}\right) \neq 0$.

Demonstração: Segue das definições (1.22), (1.21) e (1.10). 


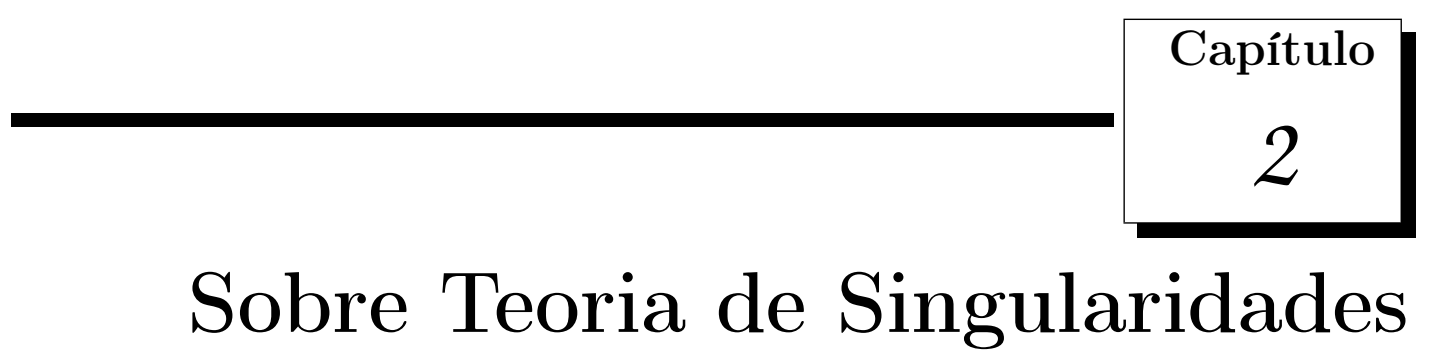

Neste capítulo são apresentadas definições e notações da Teoria de Singularidades, tais como: equivalência a direita, jatos, desdobramentos entre outros para o caso especial de funções reais. Seguimos neste capítulo a notação e resultados de Bruce e Giblin [3].

\subsection{Equivalência a direita}

Sejam $U_{i}(i=1,2)$ subconjuntos abertos da reta $\mathbb{R}, t_{i}$ um ponto de $U_{i}$ e $f_{i}: U_{i} \rightarrow \mathbb{R}$ funções suaves.

Definição 2.1. Dizemos que $f_{1}\left(e m t_{1}\right)$ e $f_{2}\left(e m t_{2}\right)$ são equivalentes à direita ( $\mathcal{R}$ equivalentes) se, existem intervalos abertos $V_{i} \subset U_{i}(i=1,2)$, com $t_{i} \in V_{i}$, um difeomorfismo $h: V_{1} \rightarrow V_{2}$ e uma constante $c$ tal que para todo $t \in V_{1}$

$$
h\left(t_{1}\right)=t_{2} \quad e \quad f_{1}(t)=f_{2}(h(t))+c,
$$

$\operatorname{com} c=f_{1}\left(t_{1}\right)-f_{2}\left(t_{2}\right)$.

Esta definição pode também ser expressa, dizendo que o seguinte diagrama comuta.

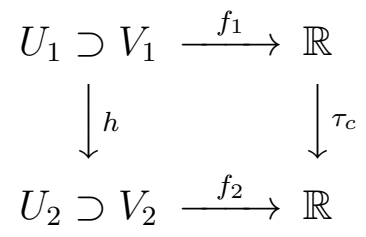

onde $\tau_{c}(x)=x-c, \forall x \in \mathbb{R}$ 
A $\mathcal{R}$-equivalência é uma relação de equivalência.

Com efeito, verifiquemos que é reflexiva, simétrica e transitiva.

(1) Reflexiva: $f$ em $t_{1}$ é $\mathcal{R}$-equivalente a $f$ em $t_{1}$, basta considerar $h$ a identidade.

(2) Simétrica: Se $f_{1}$ em $t_{1}$ é $\mathcal{R}$-equivalente a $f_{2}$ em $t_{2}$ então existe um difeomorfismo $h: V_{1} \rightarrow V_{2}$ e uma constante $c$ tal que $f_{1}(t)=f_{2}(h(t))+c$, agora consideremos o difeomorfismo $h^{-1}: V_{2} \rightarrow V_{1}$ e constante $-c$. Logo $f_{2}(s)=f_{1}\left(h^{-1}(s)\right)+(-c), \forall s \in V_{2}$.

(3) Transitiva: Se $f_{1}$ em $t_{1}$ é $\mathcal{R}$-equivalente a $f_{2}$ em $t_{2}$ então, existe um difeomorfismo $h: V_{1} \rightarrow V_{2}$ e uma constante $c_{1}$ tal que $f_{1}(t)=f_{2}(h(t))+c_{1}$ e se $f_{2}$ em $t_{2}$ é $\mathcal{R}$-equivalente a $f_{3}$ em $t_{3}$ então existe difeomorfismo $g: V_{2} \rightarrow V_{3}$ e constante $c_{2}$ tal que $f_{2}(s)=f_{3}(h(t))+c_{2}$, $\log$ o consideremos o difeomorfismo $h=g \circ h: V_{1} \rightarrow V_{3}$ e constante $c=c_{1}+c_{2}$. Portanto temos $f_{1}(t)=f_{3}(h(t))+c$ e, assim, $f_{1}$ em $t_{1}$ é $\mathcal{R}$-equivalente $f_{3}$ em $t_{3}$.

Exemplo 2.2. Uma função $F: \mathbb{R}^{2} \rightarrow \mathbb{R}$ definida numa curva parametrizada $\gamma: I \rightarrow \mathbb{R}^{2}$ e a função obtida pela mudança de parâmetro $h$ são certamente $\mathcal{R}$-equivalentes para algum $t_{1} \in I$, de forma que $t_{2}=h\left(t_{1}\right)$.

Para $t_{0} \in \mathbb{R}$, denotamos $f: \mathbb{R}, t_{0} \rightarrow \mathbb{R}$ uma função definida em alguma vizinhança de $t_{0}$. A seguir definimos o que entendemos por igualdade de duas funções deste tipo.

Dizemos que duas funções $f: \mathbb{R}, t_{0} \rightarrow \mathbb{R}$ e $g: \mathbb{R}, t_{0} \rightarrow \mathbb{R}$, são equivalentes quando, existe uma vizinhança $V$ de $t_{0}$ tal que

$$
f(t)=g(t), \quad \forall t \in V
$$

Esta é uma relação de equivalência no espaço de funções defininidas numa vizinhança de $t_{0}$. Uma classe de equivalência de tais funções é chamada de germe em $t_{0}$.

Sejam $f_{i}: \mathbb{R}, t_{i} \rightarrow \mathbb{R}, i=1,2$ duas funções, dizer que $f_{1}$ e $f_{2}$ são $\mathcal{R}$-equivalentes significa que $f_{1}$ em $t_{1}$ é $\mathcal{R}$-equivalente a $f_{2}$ em $t_{2}$.

A notação $f: \mathbb{R}, t_{0} \rightarrow \mathbb{R}, c$ quer dizer que $f$ está definido numa vizinhança de $t_{0}$ e temos $f\left(t_{0}\right)=c$.

Lema 2.3. (de Hadamard) Seja $f: \mathbb{R}, t_{0} \rightarrow \mathbb{R}$ suave, e suponha que $f^{(p)}\left(t_{0}\right)=0$ para todo $p$ com $1 \leq p \leq k$. Então existe uma função suave $f_{1}: \mathbb{R}, t_{0} \rightarrow \mathbb{R}$ tal que $f(t)=f\left(t_{0}\right)+\left(t-t_{0}\right)^{k+1} f_{1}(t)$ para todo $t$ em alguma vizinhança de $t_{0}$. Além disso se, $f^{(k+1)}\left(t_{0}\right) \neq 0$ então, $f_{1}\left(t_{0}\right) \neq 0$. 
Demonstração: É suficiente provar para o caso especial em que $t_{0}=f\left(t_{0}\right)=0$. Deste modo suponha $F: \mathbb{R}, 0 \rightarrow \mathbb{R}, 0$, suave e $F^{(p)}(0)=0$ para $1 \leq p \leq k$. Provemos que $F(t)=t^{k+1} F_{1}(t)$ para alguma função suave $F_{1}$ em todo $t$ próximo de 0 . O caso geral segue de chamarmos $F(t)=f\left(t+t_{0}\right)-f\left(t_{0}\right), f_{1}(t)=F_{1}\left(t-t_{0}\right)$. Note que $f_{1}\left(t_{0}\right) \neq 0$ e $f^{(k+1)} \neq 0$. A demonstração segue por indução sobre $k$. Para $k=0$ temos $F(0)=0$ e queremos $F(t)=t F_{1}(t)$. Agora

$$
\int_{0}^{1} \frac{d}{d u} F(t u) d u=[F(t u)]_{0}^{1}=F(t)-F(0)=F(t)
$$

Assim

$$
F(t)=\int_{0}^{1} \frac{d}{d u} F(t u) d u=\int_{0}^{1} t F^{\prime}(t u) d u=t \int_{0}^{1} F^{\prime}(t u) d u
$$

Dessa forma podemos escolher $F_{1}(t)=\int_{0}^{1} F^{\prime}(t u) d u$ que é uma função suave, suas derivadas são dadas pela diferenciação sobre o sinal de integração,

$$
F_{1}^{\prime}(t)=\int_{0}^{1} \frac{d}{d t} F^{\prime}(t u) d u
$$

A indução segue facilmente. Assumimos o resultado para $k$ e supomos $F^{(p)}(0)=0$ para $1 \leq p \leq k+1$. Então do caso $k=0, F(t)=t F_{2}(t)$ digamos, e com isto obtemos da regra de Leibniz

$$
F^{(p)}(t)=t F_{2}^{(p)}(t)+p F_{2}^{(p-1)}(t)
$$

Assim $F_{2}^{(p)}(0)=0$ para $1 \leq p \leq k$. Utilizando a hipótese de indução, $F_{2}(t)=t^{k+1} F_{1}(t)$ para uma função suave $F_{1}$, dando $F(t)=t^{k+2} F_{1}(t)$ como queriamos.

Uma versão em duas variáveis de Lema de Hadamard diz:

Se $U \subseteq \mathbb{R}^{2}$ aberto, $G: U \rightarrow \mathbb{R}$ é uma funçaõ de classe $C^{\infty}$ tal que $G(x, 0)=0, \forall(x, 0) \in U$. Então

$$
G(x, y)=y G_{1}(x, y)
$$

onde $G_{1}$ é uma função de classe $C^{\infty}$ e todo $(x, y) \in U$.

Teorema 2.4. Sejam $f: \mathbb{R}, t_{0} \rightarrow \mathbb{R}$ suave e $k \geq 0$. Suponha que $f^{(p)}\left(t_{0}\right)=0$ para todo $p$ com $1 \leq p \leq k$, e $f^{(k+1)}\left(t_{0}\right) \neq 0$. Então $f$ é $\mathcal{R}$-equivalente a $g: \mathbb{R}, 0 \rightarrow \mathbb{R}$ definida por $g(t)= \pm t^{k+1}$ onde temos + ou - de acordo com o sinal de $f^{(k+1)}\left(t_{0}\right)$ é $>0$ ou $<0$.

Demonstração: Defina $h(t)=\left(t-t_{0}\right)\left( \pm f_{1}(t)\right)^{1 /(k+1)}$ onde o sinal \pm é o sinal de $f_{1}\left(t_{0}\right)$, com $f_{1}$ dado no lema de Hadamard. Como $h\left(t_{0}\right)=0$ e $h^{\prime}\left(t_{0}\right)>0$ então, $h$ é um 
difeomorfismo em alguma vizinhança de $t_{0}$. Além disso,

$$
g(h(t))=\left(t-t_{0}\right)^{k+1} f_{1}(t)=f(t)-f\left(t_{0}\right)
$$

onde $g$ é como no teorema. Temos também que o sinal de $f_{1}\left(t_{0}\right)$ e $f^{(k+1)}\left(t_{0}\right)$ coincidem (utilizando a regra de Leibniz na fórmula do Lema de Hadamard). Dessa forma $f$ e $g$ são $\mathcal{R}$-equivalentes.

Definição 2.5. Suponhamos que $f: \mathbb{R}, t_{0} \rightarrow \mathbb{R} e ́ \mathcal{R}$-equivalente $a \pm t^{k+1}$ então, para $k \geq 0$, diremos que $f$ tem tipo $A_{k}$ em $t_{0}$, ou uma $A_{k}$ singularidade em $t_{0}$. Dessa forma tipo $A_{0}$ só diz que $f^{\prime}\left(t_{0}\right) \neq 0$. Também dizemos que $f$ tem tipo $A_{\geq k}$ quando $f^{(p)}\left(t_{0}\right)=0$ para $1 \leq p \leq k$. Ou seja, $f$ tem tipo $A_{l}$ para algum $l \geq k$ ou todas as derivadas de $f$ se anulam em $t_{0}$.

Segue do Teorema (2.4) que uma condição necessária e suficiente para $f$ ter uma $A_{k}$ singularidade em $t_{0}$ é $f^{(p)}\left(t_{0}\right)=0$ para $1 \leq p \leq k$, e $f^{(k+1)}\left(t_{0}\right) \neq 0$.

Interpretamos geometricamente a definição acima a partir do contato entre curvas.

Seja $f: \mathbb{R}, t_{0} \rightarrow \mathbb{R}$ uma função suave.

Consideremos a curva $\gamma: I \rightarrow \mathbb{R}^{2}$ definida por $\gamma(t)=(t, f(t))$ e a função $F: \mathbb{R}^{2} \rightarrow \mathbb{R}$ definida por $F\left(x_{1}, x_{2}\right)=x_{2}-f\left(t_{0}\right)$, com $F(\gamma(t))=f(t)-f\left(t_{0}\right)$. Logo $f$ tem singularidade do tipo $A_{k}$ em $t_{0}$ se, e somente se, $\gamma$ e $F^{-1}(0)$ tem $(k+1)$-ponto de contato em $t=t_{0}$, isto é, o gráfico de $f$ tem $(k+1)$-ponto de contato com a reta em $\mathbb{R}^{2}$ paralela ao eixo- $x$ passando pelo ponto $\left(t_{0}, f\left(t_{0}\right)\right)$.

Definição 2.6. Sejam $f, g: I \rightarrow \mathbb{R}$ duas funções suaves. Os gráficos de $y=f(t) e$ $y=g(t)$ tem $(k+1)$-ponto de contato em $t_{0}$, se $y_{0}=f\left(t_{0}\right)=g\left(t_{0}\right)$ e $f-g$ tem singularidade do tipo $A_{k}$ em $t_{0}$.

Observação 2.7. Seja $F: \mathbb{R}^{2},\left(x_{0}, y_{0}\right) \rightarrow \mathbb{R}, 0$ suave. Seja $\gamma: \mathbb{R}, t_{0} \rightarrow \mathbb{R}^{2}, \gamma(t)=$ $\left(\gamma_{1}(t), \gamma_{2}(t)\right)$, uma curva regular com $\gamma\left(t_{0}\right)=\left(x_{0}, y_{0}\right)$ e $\gamma_{1}^{\prime}\left(t_{0}\right) \neq 0$, então o ordem do contato entre $F^{-1}(0)$ e $\gamma$ é igual a ordem do contato entre $F^{-1}(0)$ e $\delta$ onde, $\delta(x)=$ $(x, g(x))$, para uma única $g$.

Demonstração: Seja $\pi: \mathbb{R}^{2} \rightarrow \mathbb{R}$ a primeira projeção $\pi(x, y)=x$, agora consideremos a composição $\pi \circ \gamma: \mathbb{R}, t_{0} \rightarrow \mathbb{R}$. Por diferenciação $(\pi \circ \gamma)^{\prime}\left(t_{0}\right)=\gamma_{1}^{\prime}\left(t_{0}\right) \neq 0$, logo $\pi \circ \gamma$ é um difeomorfismo local, isto é existem $V, W$ abertos de $\mathbb{R}$, com $t_{0} \in V, x_{0} \in W$ tal que $\pi \circ \gamma: V \rightarrow W$ é um difeomorfismo. Seja $\xi=(\pi \circ \gamma)^{-1}: W \rightarrow V$ considere $\delta=\gamma \circ \xi$, suponhamos $\delta(x)=(a(x), b(x))$, de onde tem-se

$$
a(x)=\pi \circ \delta(x)=(\pi \circ \gamma) \circ \xi(x)=(\pi \circ \gamma) \circ(\pi \circ \gamma)^{-1}(x)=x
$$


Considerando $g: W \rightarrow \mathbb{R}, g(x)=b(x), \forall x \in W$, obtemos $\delta(x)=(x, g(x))$. Observemos que para $t \in V, F(\gamma(t))=F(\gamma(\xi(x)))=F(\delta(x))$, logo por derivação $(F \circ \delta)^{\prime}(x)=(F \circ \gamma)^{\prime}(t) \xi^{\prime}(x)$, onde $t=\xi(x)$, $(F \circ \delta)^{\prime \prime}(x)=(F \circ \gamma)^{\prime \prime}(t) \xi^{\prime}(x)^{2}+(F \circ \gamma)^{\prime}(t) \xi^{\prime \prime}(x)$, e assim obtemos

$$
(F \circ \delta)^{(n)}(x)=(F \circ \gamma)^{(n)}(t) \xi^{\prime}(x)^{n}+\text { termos envolvendo derivadas de }(F \circ \gamma) \text { e } \xi
$$

Se $\xi^{\prime}\left(x_{0}\right) \neq 0$, desde a relação acima pode-se concluir que o contato de $F^{-1}(0)$ e $\gamma$ em $t_{0}$, pode ser substituido pelo contato de $F^{-1}(0)$ e $\delta$ em $x_{0}$. Vejamos que $\xi^{\prime}\left(x_{0}\right) \neq 0$, de fato, como $(\pi \circ \gamma) \circ \xi(x)=x$, aplicando regra da cadeia temos $(\pi \circ \gamma)^{\prime}\left(t_{0}\right) \xi^{\prime}\left(x_{0}\right)=1$, isto é $\gamma_{1}^{\prime}\left(t_{0}\right) \xi^{\prime}\left(x_{0}\right)=1$, de onde concluimos $\xi^{\prime}\left(x_{0}\right) \neq 0$.

Observação 2.8. Seja $F: \mathbb{R}^{2},\left(x_{0}, y_{0}\right) \rightarrow \mathbb{R}$ suave e suponhamos $\frac{\partial F}{\partial y}\left(x_{0}, y_{0}\right) \neq 0$. Seja $\gamma: \mathbb{R}, t_{0} \rightarrow \mathbb{R}^{2}, \gamma(t)=\left(\gamma_{1}(t), \gamma_{2}(t)\right)$, uma curva regular, com $\gamma\left(t_{0}\right)=\left(x_{0}, y_{0}\right)$, então mantendo o mesmo contato, $F$ pode ser substituido por $G$ onde $G(x, y)=y-f(x)$ para uma única $f$.

Demonstração: Sem perda de geralidade podemos supor que $F\left(x_{0}, y_{0}\right)=0$, como $F$ é suave e $\frac{\partial F}{\partial y}\left(x_{0}, y_{0}\right) \neq 0$, segue pelo Teorema da Função Implícita que existe um aberto $\mathrm{U}$ contendo $x_{0}$ e uma função suave $f: U \rightarrow \mathbb{R}$ tal que $F(x, f(x))=0, \forall x \in U$. Agora consideremos a função $H: \mathbb{R}^{2},\left(x_{0}, y_{0}\right) \rightarrow \mathbb{R}$ definida por

$$
H(x, y)=F(x, f(x)-y)
$$

como $H(x, 0)=F(x, f(x))=0$, pelo Lema de Hadamard, existe $G_{1}: \mathbb{R}^{2},\left(x_{0}, y_{0}\right) \rightarrow \mathbb{R}$ suave tal que $H(x, y)=y G_{1}(x, y)$, daí segue

$$
F(x, y)=H(x, y-f(x))=(y-f(x)) G_{1}(x, y-f(x))
$$

assim consideremos $F_{1}(x, y)=G_{1}(x, y-f(x))$. Aplicando o Lema (1.14) para as funções $F$ e $G \operatorname{com} G(x, y)=y-f(x)$ segue o resultado.

Corolário 2.9. Seja $F: \mathbb{R}^{2},\left(x_{0}, y_{0}\right) \rightarrow \mathbb{R}$ suave e suponhamos $\frac{\partial F}{\partial y}\left(x_{0}, y_{0}\right) \neq 0$. Seja $\gamma: \mathbb{R}, t_{0} \rightarrow \mathbb{R}^{2}, \gamma(t)=\left(\gamma_{1}(t), \gamma_{2}(t)\right)$, uma curva regular, com $\gamma\left(t_{0}\right)=\left(x_{0}, y_{0}\right)$ e $\gamma_{1}^{\prime}\left(t_{0}\right) \neq 0$, então mantendo o mesmo contato, $F$ pode ser substituido por $G$ onde $G(x, y)=y-f(x)$ para uma única $f$, e $\gamma$ pode ser substituido por $\delta$ onde $\delta(t)=(t, g(t))$, para uma única $g$.

Corolário 2.10. Seja $F: \mathbb{R}^{2},\left(x_{0}, y_{0}\right) \rightarrow \mathbb{R}$ suave e suponhamos $\frac{\partial F}{\partial y}\left(x_{0}, y_{0}\right) \neq 0$. Seja $\gamma: \mathbb{R}, t_{0} \rightarrow \mathbb{R}^{2}, \gamma(t)=\left(\gamma_{1}(t), \gamma_{2}(t)\right)$, uma curva regular, com $\gamma\left(t_{0}\right)=\left(x_{0}, y_{0}\right)$ e $\gamma_{1}^{\prime}\left(t_{0}\right) \neq 0$, 
então $\gamma$ e $F^{-1}(0)$ tem $k$-ponto de contato em $t_{0}$ se, e somente se, a função $f-g$ tem tipo $A_{k-1}$ em $x_{0}$, onde $f$ e $g$ são as funções obtidas no corolário acima.

Assim, quando duas curvas são representadas localmente por equações $y=f(x), y=g(x)$ (onde $f\left(x_{0}\right)=g\left(x_{0}\right)$ ), o ordem do contato é medido pelo tipo de singularidade de $f-g$ em $x_{0}$.

Demonstração: Sejam $G(x, y)=y-f(x)$ e $\delta(x)=(x, g(x))$ como nas proposições acima, $\log 0 G \circ \delta(x)=G(x, g(x))=f(x)-g(x)$, assim $\gamma$ e $F^{-1}(0)$ tem $k$-ponto de contato em $t_{0}$ se, e somente se, a função $f-g$ tem tipo $A_{k-1}$ em $x_{0}$.

\subsection{Jatos de funções}

Seja $f: \mathbb{R}, t_{0} \rightarrow \mathbb{R}$ função suave, a série de Taylor de $f$ em $t_{0}$ pode ser escrita na forma

$$
f\left(t_{0}\right)+t f^{\prime}\left(t_{0}\right)+\frac{t^{2}}{2 !} f^{\prime \prime}\left(t_{0}\right)+\frac{t^{3}}{3 !} f^{\prime \prime \prime}\left(t_{0}\right)+\ldots
$$

onde expandimos $f\left(t+t_{0}\right)$ em vez de $f(t)$, de modo que $t$ está próximo de 0 em vez de $t_{0}$.

Definição 2.11. Seja $k \geq 1$ um inteiro. O k-jato de $f$ em $t_{0}$ é o polinômio

$$
j^{k} f\left(t_{0}\right)=t f^{\prime}\left(t_{0}\right)+\frac{t^{2}}{2 !} f^{\prime \prime}\left(t_{0}\right)+\ldots+\frac{t^{k}}{k !} f^{(k)}\left(t_{0}\right)
$$

obtido do truncamento da série de Taylor no grau $k$ e excluindo o termo constante $f\left(t_{0}\right)$.

Dois $k$-jatos são iguais quando são idênticos como polinômios. As vezes incluimos o termo constante no jato. Nos referimos a $f\left(t_{0}\right)+j^{k} f\left(t_{0}\right)$ como o $k$-jato com termo constante de $f$ em $t_{0}$.

Exemplo 2.12. Por um cálculo simples $f$ tem tipo $A_{k}$ em $t_{0}$ se, e somente se, $j^{k} f\left(t_{0}\right)=0$ e $j^{k+1} f\left(t_{0}\right) \neq 0$ (assumimos $k \geq 1$ ) mas pensando em $j^{0} f\left(t_{0}\right)=0$ como 0 , isto vale para $k=0$ também. Além disso $f$ tem tipo $A_{m}$ para algum $m \geq k$ se, e somente se, $j^{k} f\left(t_{0}\right)=0$ e alguma derivada de $f$ é não nula em $t_{0}$.

Observemos que nem todas as funções são do tipo $A_{k}$ para algum $k$, um exemplo de tais funções é a função $f: \mathbb{R} \rightarrow \mathbb{R}$ definida por $f(t)=\exp (-1 / t)$ para $t>0$ e $f(t)=0$ para $t \leq 0$. Esta função é denominada função flat em 0 , esta função satisfaz que todas as derivadas são nulas, ou seja $f^{(p)}(0)=0$ para todo $p \geq 1$. 
Lema 2.13. Seja $f: \mathbb{R}, t_{0} \rightarrow \mathbb{R}$ função suave e seja $k$ um inteiro $\geq 0$, então existe uma função $f_{1}: \mathbb{R}, t_{0} \rightarrow \mathbb{R}$ tal que

$$
f(t)=\sum_{p=0}^{k} a_{p}\left(t-t_{0}\right)^{p}+\left(t-t_{0}\right)^{k+1} f_{1}(t)
$$

para t numa vizinhança de $t_{0}$, onde $a_{p}=\frac{f^{(p)}\left(t_{0}\right)}{p !}$

Demonstração: Consideremos a função $g: \mathbb{R}, t_{0} \rightarrow \mathbb{R}$ definida por

$$
g(t)=f(t)-\sum_{p=0}^{k} a_{p}\left(t-t_{0}\right)^{p}
$$

por diferenciação $g^{(l)}\left(t_{0}\right)=0, \forall l=0,1, \ldots, k$.

Logo pelo Lema de Hadamard, existe uma função suave $f_{1}: \mathbb{R}, t_{0} \rightarrow \mathbb{R}$ tal que

$$
g(t)-g\left(t_{0}\right)=\left(t-t_{0}\right)^{k+1} f_{1}(t)
$$

de onde conclui-se pois $g\left(t_{0}\right)=0$.

\subsection{Desdobramentos}

Uma família de funções contendo $f$ é chamada de desdobramento de $f$, o nome de desdobramento é porque a família $F$ se "desdobra" e revela todas estas funções que são próximas de $f$.

Seja $F: \mathbb{R} \times \mathbb{R}^{r},\left(t_{0}, x_{0}\right) \rightarrow \mathbb{R}$ uma função suave. Podemos considerar $\mathrm{F}$ como uma família a $r$-parâmetros de funções $F_{x}: \mathbb{R}, t_{0} \rightarrow \mathbb{R}$ ou como uma família a 1-parâmetro de funções $F_{t}: \mathbb{R}^{r}, x_{0} \rightarrow \mathbb{R}$. Escrevemos $f=F_{x_{0}}: \mathbb{R}, t_{0} \rightarrow \mathbb{R}$ a função definida por $f(t)=F\left(t, x_{0}\right)$.

Definição 2.14. F como acima é chamada desdobramento a r-parâmetros de $f$.

Sabemos que uma função $f: \mathbb{R}, t_{0} \rightarrow \mathbb{R}$ tendo uma singularidade $A_{k}$ em $t_{0}$ pode ser reduzida, em uma vizinhança de $t_{0}$ para uma das formas $g(t)= \pm t^{k+1}$, isto é existe difeomorfismo local $h: \mathbb{R}, t_{0} \rightarrow \mathbb{R}, 0$ e uma constante $c$ tal que $f(t)=g(h(t))+c$, ou seja existem $U_{1}, U_{2}$ intervalos abertos de $\mathbb{R}$ tal que o seguinte diagrama comuta

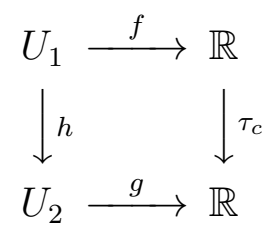


Agora suponhamos que $F: \mathbb{R} \times \mathbb{R}^{r},\left(t_{0}, x_{0}\right) \rightarrow \mathbb{R}$ é um desdobramento $f$. A seguir reduziremos $F$ a uma forma normal $G$ via mudança de variáveis nos seguintes quatro itens.

1. $F$ pode ser reduzida como $f$, isto é, defina:

$$
\bar{F}(t, x)=F\left(h^{-1}(t), x\right)-c
$$

tal que $\bar{F}\left(t, x_{0}\right)=g(t)$, para $t$ próximo a 0 . Consideremos só o caso $g(t)=t^{k+1}$.

2. Do Lema (2.13), para cada $x \in \mathbb{R}$ escrevemos $\bar{F}(t, x)$ na seguinte forma

$$
\bar{F}(t, x)=b_{0}(x)+b_{1}(x) t+\ldots+b_{k+1}(x) t^{k+1}+t^{k+2} \theta(t, x)
$$

para funções unicamente determinadas $b_{i}: \mathbb{R}^{r}, x_{0} \rightarrow \mathbb{R}$ e $\theta: \mathbb{R} \times \mathbb{R}^{r},\left(0, x_{0}\right) \rightarrow \mathbb{R}$. Por outro lado $\bar{F}\left(t, x_{0}\right)=t^{k+1}$, daí $b_{i}\left(x_{0}\right)=0$ para $0 \leq i \leq k$, e $b_{k+1}\left(x_{0}\right)=1$.

3. Por uma mudança de variável eliminamos $t^{k+2} \theta(t, x)$, reduzimos $b_{k+1}(x)$ a 1 e tiramos o termo em $t^{k}$. Para isto substituimos $t$ por $t-\frac{1}{3} \lambda$ (transformação de Tschirnhaus's). Assim obtemos novas funções $b_{i}$ e $c$ e

$$
\bar{F}\left(a_{1}(t, x), x\right)=c(x)+b_{1}(x) t+\ldots+b_{k-1}(x) t^{k-1}+t^{k+1}
$$

onde $a_{1}: \mathbb{R} \times \mathbb{R}^{r},\left(0, x_{0}\right) \rightarrow \mathbb{R}$ é suave, $a_{1}\left(t, x_{0}\right)=t$ para todo $t$ próximo de $0 \mathrm{e}$ $b_{i}\left(x_{0}\right)=0$ para $i=1, \ldots, k-1$ e $c\left(x_{0}\right)=0$.

Agora definimos a função $G: \mathbb{R} \times \mathbb{R}^{k-1} \rightarrow \mathbb{R}$ dada por

$$
G\left(t, u_{1}, \ldots, u_{k-1}\right)=u_{1} t+u_{2} t^{2}+\ldots+u_{k-1} t^{k-1}+t^{k+1} .
$$

4. Seja $a_{1} \times i d: \mathbb{R} \times \mathbb{R}^{r},\left(0, x_{0}\right) \rightarrow \mathbb{R} \times \mathbb{R}^{r}$ dada por $\left(a_{1} \times i d\right)(t, x)=\left(a_{1}(t, x), x\right)$. Então temos que $a_{1} \times i d$ é um difeomorfismo local em $\left(0, x_{0}\right)$, usando $a_{1}\left(t, x_{0}\right)=t$. Definimos $a: \mathbb{R} \times \mathbb{R}^{r},\left(0, x_{0}\right) \rightarrow \mathbb{R}$ como $a \times i d=\left(a_{1} \times i d\right)^{-1}$. Então pode-se escrever:

$$
\bar{F}(t, x)=G(a(t, x), b(x))+c(x) .
$$

O que completa a redução de $\bar{F}$ à uma forma normal $G$ via mudança de variáveis dadas por $a, b, c$. 


\subsection{Desdobramento (p)versal}

Definição 2.15. Seja $G: \mathbb{R} \times \mathbb{R}^{s},\left(t_{0}, y_{0}\right) \rightarrow \mathbb{R}$ um desdobramento a s-parâmetros da função $g: \mathbb{R}, t_{0} \rightarrow \mathbb{R}$.

Sejam

$$
a: \mathbb{R} \times \mathbb{R}^{r},\left(t_{0}, y_{0}\right) \rightarrow \mathbb{R}
$$

função suave que satisfaz $a\left(t, x_{0}\right)=t$ para $t$ próximo a $t_{0}$,

$$
b: \mathbb{R}^{r}, x_{0} \rightarrow \mathbb{R}^{s}, y_{0}
$$

função suave $e$

$$
c: \mathbb{R}^{r}, x_{0} \rightarrow \mathbb{R}^{s}
$$

função suave. Então o desdobramento

$$
F: \mathbb{R} \times \mathbb{R}^{r},\left(t_{0}, x_{0}\right) \rightarrow \mathbb{R}
$$

definido como

$$
F(t, x)=G(a(t, x), b(x))+c(x)
$$

é dito ser (p)induzido de G.

Note que se $F$ é um desdobramento de $f$, então $f(t)=F\left(t, x_{0}\right)$. Como $F\left(t, x_{0}\right)=$ $G\left(a\left(t, x_{0}\right), b\left(x_{0}\right)\right)+c\left(x_{0}\right)$ e $G\left(t, y_{0}\right)=g(t)$ então $f(t)=g(t)+c\left(x_{0}\right)$, daí $F$ é um desdobramento de $f$ que é igual a $g$ a menos de uma constante aditiva.

Definição 2.16. Seja $G: \mathbb{R} \times \mathbb{R}^{s},\left(t_{0}, y_{0}\right) \rightarrow \mathbb{R}$ um desdobramento a s-parâmetros da função $g=\mathbb{R}, t_{0} \rightarrow \mathbb{R}$. G é chamado desdobramento (p)versal de $g$ em $t_{0}$, se qualquer desdobramento de gé (p)induzido de $G$.

Teorema 2.17. O desdobramento $G: \mathbb{R} \times \mathbb{R}^{k-1},(0,0) \rightarrow \mathbb{R}$ dado por

$$
G(t, x)= \pm t^{k+1}+x_{1} t+x_{2} t^{2}+\ldots+x_{k-1} t^{k-1}
$$

é um desdobramento (p)versal de $g(t)= \pm t^{k+1}$ em $t_{0}=0$.

Demonstração: Decorre desde as relações (2.2) e (2.3)

Este desdobramento é chamado miniversal desde que o número de parâmetros do desdobramento seja mínimo. 
Exemplo 2.18. Sejam $G: \mathbb{R} \times \mathbb{R} \rightarrow \mathbb{R}$ e $H: \mathbb{R} \times \mathbb{R}^{2} \rightarrow \mathbb{R}$ definidas por

$$
G\left(t, x_{1}\right)=t^{3}+x_{1} t \text { e } H\left(t, x_{1}, x_{2}\right)=t^{3}+x_{1} t+x_{2} t^{2}
$$

$G$ e $H$ assim definidas são desdobramentos da função $f: \mathbb{R} \rightarrow \mathbb{R}, f(t)=t^{3}$. Além disso, $G$ é induzido de $H$ e reciprocamente $H$ é induzido de $G$.

Primeiro induzimos $G$ desde $H$, para isto basta considerar $G\left(t, x_{1}\right)=H\left(t, x_{1}, 0\right)$, ou seja, $G\left(t, x_{1}\right)=H\left(a\left(t, x_{1}\right), b\left(x_{1}\right)\right)+c\left(x_{1}\right)$, onde $a\left(t, x_{1}\right)=t, b\left(x_{1}\right)=\left(x_{1}, 0\right)$ e $c\left(x_{1}\right)=0$. Agora induzimos $H$ desde $G$. Como primeiro passo eliminamos o termo $t^{2}$ considerando a mudança $t \mapsto t-\frac{1}{3} x_{2}$ :

$$
\begin{aligned}
H\left(t-\frac{1}{3} x_{2}, x_{1}, x_{2}\right) & =\left(t-\frac{1}{3} x_{2}\right)^{3}+x_{1}\left(t-\frac{1}{3} x_{2}\right)+x_{2}\left(t-\frac{1}{3} x_{2}\right)^{2} \\
& =t^{3}+\left(x_{1}-\frac{1}{3} x_{2}^{2}\right) t+\left(\frac{2}{27} x_{2}^{3}-\frac{1}{3} x_{1} x_{2}\right) \\
& =G\left(t, x_{1}-\frac{1}{3} x_{2}^{2}\right)+\left(\frac{2}{27} x_{2}^{3}-\frac{1}{3} x_{1} x_{2}\right) .
\end{aligned}
$$

Portanto, $H\left(t, x_{1}, x_{2}\right)=G\left(t+\frac{1}{3} x_{2}, x_{1}-\frac{1}{3} x_{2}^{2}\right)+\left(\frac{2}{27} x_{2}^{3}-\frac{1}{3} x_{1} x_{2}\right)$ e o resultado segue para $a\left(t, x_{1}, x_{2}\right)=t+\frac{1}{3} x_{2}$.

Teorema 2.19. (Critério de (p)versalidade) Seja $F: \mathbb{R} \times \mathbb{R}^{r},\left(t_{0}, x_{0}\right) \rightarrow \mathbb{R}$ um desdobramento a r-parâmetros de $f: \mathbb{R}, t_{0} \rightarrow \mathbb{R}$ e suponhamos que $f$ tem singularidade de tipo $A_{k}, k \geq 1$ em $t_{0}$. Então $F$ é (p)versal se, e somente se, todo polinomio real $p(t) d e$ grau $\leq k-1$ e sem termo constante pode ser escrito na seguinte forma:

$$
p(t)=\sum_{i=1}^{r} c_{i} j^{k-1}\left(\frac{\partial F}{\partial x_{i}}\left(t, x_{0}\right)\right)\left(t_{0}\right)
$$

para constantes reais $c_{i}$, onde $j^{k-1}$ denota $o(k-1)$-jato.

Demonstração: $\operatorname{Ver}([3]$ p. 141).

O seguinte teorema esta enunciado sem demostração no livro de Bruce e Giblin ([3] p. 141). Este teorema fornece outro critério para (p)versalidade.

Teorema 2.20. (Criterio matricial de (p)versalidade) Seja $F: \mathbb{R} \times \mathbb{R}^{r},\left(t_{0}, x_{0}\right) \rightarrow \mathbb{R}$, um desdobramento a r-parâmetros de $f: \mathbb{R}, t_{0} \rightarrow \mathbb{R}$ e suponhamos que $f$ tem singularidade de tipo $A_{k}, k \geq 1$ em $t_{0}$, e seja

$$
j^{k-1}\left(\frac{\partial F}{\partial x_{i}}\left(t, x_{0}\right)\right)\left(t_{0}\right)=\alpha_{1, i} t+\alpha_{2, i} t^{2}+\ldots+\alpha_{k-1, i} t^{k-1}
$$


para $i=1, \ldots, r$. Então $F$ é $(p)$ versal se, e somente se, a matriz $(k-1) \times r$ dos coeficientes $\alpha$ tem posto $k-1$.

\subsubsection{Conjunto singular - conjunto bifurcação}

Definição 2.21. Seja $F: \mathbb{R} \times \mathbb{R}^{r},\left(t_{0}, x_{0}\right) \rightarrow \mathbb{R}$ um desdobramento a r-parâmetros de $f$. $O$ conjunto singular $S_{F}$ de $F$ é o conjunto :

$$
S_{F}=\left\{(t, x) \in \mathbb{R} \times \mathbb{R}^{r}: \frac{\partial F}{\partial t}=0 \text { em }(t, x)\right\}
$$

ou seja, o conjunto singular consiste de pares $(t, x)$ tal que $F_{x}$ é singular em $t$.

$O$ conjunto de bifurcação $\mathcal{B}_{F}$ de $F$ é o conjunto:

$$
\mathcal{B}_{F}=\left\{x \in \mathbb{R}^{r}: \exists t \text { tal que } \frac{\partial F}{\partial t}=\frac{\partial^{2} F}{\partial t^{2}}=0 \text { em }(t, x)\right\}
$$

Se $x \in \mathcal{B}_{F} e \frac{\partial F}{\partial t}=\frac{\partial^{2} F}{\partial t^{2}}=0$ em $(t, x)$, dizemos que $t$ corresponde a $x$.

Observemos que se $F$ é um desdobramento (p)versal a $r$-parâmetros de $f$ então $S_{F}$ é uma $r$-variedade parametrizada em $\mathbb{R}^{r+1}$.

De fato, suponhamos que $F: \mathbb{R} \times \mathbb{R}^{r},\left(t_{0}, x_{0}\right) \rightarrow \mathbb{R}$ é um desdobramento (p) versal a $r$-parâmetros de $f: \mathbb{R}, t_{0} \rightarrow \mathbb{R}$ e $\left(t_{0}, x_{0}\right) \in S_{F}$ e consideremos a função

$$
\begin{aligned}
H: \mathbb{R} \times \mathbb{R}^{r},\left(t_{0}, x_{0}\right) & \rightarrow \mathbb{R} \\
(t, x) & \mapsto H(t, x)=\frac{\partial F}{\partial t}(t, x)
\end{aligned}
$$

Como $F$ é desdobramento (p)versal, então pelo critério (p)versal e pela equação (2.4) o polinômio

$$
\sum_{i=1}^{r} c_{i} \frac{\partial H}{\partial x_{i}}\left(t_{0}, x_{0}\right) t=\sum_{i=1}^{r} c_{i} \frac{\partial^{2} F}{\partial t \partial x_{i}}\left(t_{0}, x_{0}\right) t
$$

é diferente de zero. Portanto $\left(t_{0}, x_{0}\right)$ é um ponto regular de $H$, e assim $S_{F}$ é uma $r$ variedade parametrizada em $\mathbb{R}^{r+1}$ numa vizinhança $U_{1}$ de $\left(t_{0}, x_{0}\right)$.

Notemos também que se $\left(t_{0}, x_{0}\right) \in S_{F}$ então

$$
\frac{\partial f}{\partial t}\left(t_{0}\right)=\frac{\partial F_{x_{0}}}{\partial t}\left(t_{0}\right)=\frac{\partial F}{\partial t}\left(t_{0}, x_{0}\right)=0,
$$


assim $f$ tem singularidade do tipo $A_{\geq 1}$ em $t_{0}$.

Se $F$ e $G$ são dois desdobramentos (p)versais a $r$-parâmetros quaisquer de $f\left(\right.$ em $t_{0}$ ) e $g\left(\right.$ em $\left.t_{1}\right)$, respectivamente, ambos do tipo $A_{k}(k \geq 1)$. Então existem vizinhanças $U$ de $\left(t_{0}, x_{0}\right)$ e $V$ de $\left(t_{1}, u_{0}\right)$ em $\mathbb{R} \times \mathbb{R}^{r}$ tal que $S_{F} \cap U$ e $S_{G} \cap V$ são $r$-variedades parametrizadas.

Proposição 2.22. (Unicidade dos conjuntos de bifurcação) Sejam $F$ e $G$ dois desdobramentos (p)versais a r-parâmetros quaisquer de $f$ (em $\left.t_{0}\right)$ e $g$ (em $t_{1}$ ), respectivamente, ambos do tipo $A_{k}(k \geq 1)$, então existem vizinhanças $U$ e $V$ e um difeomorfismo $\phi: U \rightarrow V$ na forma $\phi(t, x)=(a(t, x), b(x))$, onde $a\left(t_{0}, x_{0}\right)=t_{1}, b\left(x_{0}\right)=u_{0} e$

1. $\phi\left(S_{F} \cap U\right)=S_{G} \cap V$

2. $b$ é um difeomorfismo de $\pi(U)$ a $\pi(V)$, onde cada $\pi$ é projeção dos parâmetros de desdobramento, e $b\left(\mathcal{B}_{F} \cap \pi(U)\right)=\mathcal{B}_{G} \cap \pi(V)$.

Demonstração: $\operatorname{Ver}([3]$, p. 145).

Devido ao Teorema (2.22) os conjuntos bifurcação de $F$ e $G$ são localmente difeomorfos quando $f$ e $g$ possuem singularidade de tipo $A_{k}$.

\subsubsection{Desdobramento (p)versal de $A_{2}(r \geq 1)$}

Consideremos dois casos:

Para $r=1$, o desdobramento $G: \mathbb{R} \times \mathbb{R},(0,0) \rightarrow \mathbb{R}$ dado por $G(t, x)= \pm t^{3}+x t$ é um desdobramento (p)versal de $g(t)= \pm t^{3}$ em $t_{0}=0$. Por outro lado considerando a mudança $t \mapsto-t$, obtemos que $t^{3}$ e $-t^{3}$ são $\mathcal{R}$-equivalentes, com isto só consideremos o desdobramento (p)versal $F: \mathbb{R} \times \mathbb{R},(0,0) \rightarrow \mathbb{R}$ de $f(t)= \pm t^{3}$ o qual é definido por $F(t, x)=t^{3}+x t$.

O conjunto singular é

$$
S_{F}=\left\{(t, x) \in \mathbb{R} \times \mathbb{R}: 3 t^{2}+x=0\right\},
$$

e o conjunto bifurcação

$$
\mathcal{B}_{F}=\left\{x \in \mathbb{R}: \text { para algum } t, 3 t^{2}+x=6 t=0\right\},
$$

é exatamente $\{0\}$ isto é, um ponto de $\mathbb{R}$. 
Para $r>1, \tilde{F}: \mathbb{R} \times \mathbb{R}^{r},(0,0) \rightarrow \mathbb{R}$ definido por $\tilde{F}\left(t, x_{1}, x_{2} \ldots, x_{r}\right)=t^{3}+x_{1} t$ é um desdobramento (p)versal $r$-parâmetros de $f$, o conjunto singular é

$$
S_{\tilde{F}}=\left\{\left(t, x_{1}, \ldots, x_{r}\right) \in \mathbb{R} \times \mathbb{R}: 3 t^{2}+x_{1}=0\right\}
$$

e o conjunto bifurcação

$$
\mathcal{B}_{\tilde{F}}=\left\{\left(x_{1}, \ldots, x_{r}\right) \in \mathbb{R}^{r}: \text { para algum } t, 3 t^{2}+x_{1}=6 t=0\right\},
$$

é exatamente $\{0\} \times \mathbb{R}^{r-1}$.

Então localmente o conjunto bifurcação de um desdobramento (p)-versal a $r$-parâmetros de uma singularidade $A_{2}$ é um espaço linear de dimensão $r-1$ em $\mathbb{R}^{r}$, isto é, uma variedade $(r-1)$ local parametrizada em $\mathbb{R}^{r}$.

\subsubsection{Desdobramento (p)versal de $A_{3}(r \geq 2)$}

Seja $F: \mathbb{R} \times \mathbb{R}^{r},(0,0) \rightarrow \mathbb{R},(r \geq 2)$ definido como

$$
F\left(t, x_{1}, x_{2}, \ldots, x_{r}\right)=\frac{1}{4} t^{4}+x_{1} t+\frac{1}{2} x_{2} t^{2} .
$$

$F$ é um desdobramento (p)-versal de $f(t)=\frac{1}{4} t^{4}$ em $t_{0}=0$. O conjunto bifurcação é

$$
\mathcal{B}_{F}=\left\{\left(x_{1}, \ldots, x_{r}\right) \in \mathbb{R}^{r}: \text { para algum } t, t^{3}+x_{1}+x_{2} t=3 t^{2}+x_{2}=0\right\} .
$$

$\operatorname{Logo}\left(x_{1}, \ldots, x_{r}\right) \in \mathcal{B}_{F} \Leftrightarrow t^{3}+x_{1}+x_{2} t=0$ e $3 t^{2}+x_{2}=0$,

$$
\begin{aligned}
& \Leftrightarrow x_{2}=-3 t^{2} \text { e } x_{1}=2 t^{3}, \\
& \Leftrightarrow 27 x_{1}^{2}+4 x_{2}^{3}=0,
\end{aligned}
$$

portanto

$$
\mathcal{B}_{F}=\left\{\left(x_{1}, \ldots, x_{r}\right) \in \mathbb{R}^{r}: 27 x_{1}^{2}+4 x_{2}^{3}=0\right\} .
$$

Logo, considerando o difeomorfismo

$$
\xi: \mathcal{B}_{F} \rightarrow C \times \mathbb{R}^{r-2}
$$

definido por

$$
\left(x_{1}, x_{2}, x_{3}, \ldots, x_{r}\right) \mapsto\left(x_{1},-\frac{\sqrt[3]{4}}{3} x_{2}, x_{3}, \ldots, x_{r}\right),
$$

obtemos que $\mathcal{B}_{F}$ é difeomorfo com $C \times \mathbb{R}^{r-2}$, onde $C=\left\{\left(x_{1}, x_{2}\right) / x_{1}^{2}=x_{2}^{3}\right\}$. 


\subsection{Desdobramento versal}

Definição 2.23. Seja $G: \mathbb{R} \times \mathbb{R}^{s},\left(t_{0}, y_{0}\right) \rightarrow \mathbb{R}$ um desdobramento a s-parâmetros da função $g: \mathbb{R}, t_{0} \rightarrow \mathbb{R}$.

Sejam

$$
a: \mathbb{R} \times \mathbb{R}^{r},\left(t_{0}, y_{0}\right) \rightarrow \mathbb{R}
$$

função suave que satisfaz $a\left(t, x_{0}\right)=t$, para $t$ numa vizinhança de $t_{0} e$

$$
b: \mathbb{R}^{r}, x_{0} \rightarrow \mathbb{R}^{s}, y_{0}
$$

função suave. Então o desdobramento

$$
F: \mathbb{R} \times \mathbb{R}^{r},\left(t_{0}, x_{0}\right) \rightarrow \mathbb{R}
$$

definido como

$$
F(t, x)=G(a(t, x), b(x))
$$

é dito ser induzido de G.

Definição 2.24. Seja $G: \mathbb{R} \times \mathbb{R}^{s},\left(t_{0}, y_{0}\right) \rightarrow \mathbb{R}$ um desdobramento a s-parâmetros da função $g: \mathbb{R}, t_{0} \rightarrow \mathbb{R}, G$ é chamado desdobramento versal de $g$ em $t_{0}$, se qualquer desdobramento de g é induzido de $G$.

Teorema 2.25. O desdobramento $G: \mathbb{R} \times \mathbb{R}^{k} \rightarrow \mathbb{R}$ dado por

$$
G\left(t, x_{1}, \ldots, x_{k}\right)= \pm t^{k+1}+x_{1}+x_{2} t+\ldots+x_{k} t^{k-1}
$$

é um desdobramento versal de $g(t)= \pm t^{k+1}$ em $t_{0}=0$, sendo miniversal quando tem o número mínimo de parâmetros, $k$, para uma singularidade do tipo $A_{k}$.

Demonstração: Ver ([3], p.149).

Teorema 2.26. (Critério para versalidade) Seja $F: \mathbb{R} \times \mathbb{R}^{r},\left(t_{0}, x_{0}\right) \rightarrow \mathbb{R}$, um desdobramento a r-parâmetros de $f: \mathbb{R}, t_{0} \rightarrow \mathbb{R}$ e suponhamos que $f$ tem singularidade de tipo $A_{k}, k \geq 1$ em $t_{0}$.

Então $F$ é versal se, e somente se, qualquer polinômio real $p(t)$ de grau $\leq k-1$ pode ser escrito como uma combinação linear real de $(k-1)$-jatos com termo constante $\frac{\partial F}{\partial x_{i}}\left(t, x_{0}\right)$ em $t_{0}, i=1, \ldots, r$,

$$
p(t)=\sum_{i=1}^{r} c_{i}\left[\frac{\partial F}{\partial x_{i}}\left(t_{0}, x_{0}\right)+j^{k-1}\left(\frac{\partial F}{\partial x_{i}}\left(t, x_{0}\right)\right)\left(t_{0}\right)\right]
$$


para constantes reais $c_{i}$, onde $j^{k-1}$ denota $o(k-1)$-jato.

Demonstração: Ver ([3], p. 149).

Teorema 2.27. (Critério matricial para versalidade) Seja $F: \mathbb{R} \times \mathbb{R}^{r},\left(t_{0}, x_{0}\right) \rightarrow \mathbb{R}$, um desdobramento a r-parâmetros de $f: \mathbb{R}, t_{0} \rightarrow \mathbb{R}$ e suponhamos que $f$ tem singularidade de tipo $A_{k}, k \geq 1$ em $t_{0}$. Escrevemos o $(k-1)$-jato com constante de $\frac{\partial F}{\partial x_{i}}\left(t, x_{0}\right)$ em $t_{0}$ como

$$
\alpha_{0, i}+\alpha_{1, i} t+\alpha_{2, i} t^{2}+\ldots+\alpha_{k-1, i} t^{k-1}
$$

para $i=1, \ldots, r$. Então $F$ é versal se, e somente se, a matriz $k \times r$ dos coeficientes $\left(\alpha_{j, i}\right)$ tem posto $k$ (isto é possivel unicamente se $r \geq k$ ).

\subsubsection{Conjunto discriminante}

Seja $F: \mathbb{R} \times \mathbb{R}^{r},\left(t_{0}, x_{0}\right) \rightarrow \mathbb{R}$ um desdobramento de $f: \mathbb{R}, t_{0} \rightarrow \mathbb{R}$ e suponhamos que $f$ tem singularidade de tipo $A_{k}, k \geq 1$ em $t_{0}$.

O conjunto de zeros $M_{F}$ de $F$ é

$$
M_{F}=\left\{(t, x) \in \mathbb{R} \times \mathbb{R}^{r}: F(t, x)=0\right\}
$$

e o conjunto discriminante $\mathcal{D}_{F}$ de $F$ é

$$
\mathcal{D}_{F}=\left\{x \in \mathbb{R}^{r}: \text { existe } t \in \mathbb{R} \text { com } F=\frac{\partial F}{\partial t}=0 \text { em }(t, x)\right\}
$$

De modo análogo com desdobramentos (p)versais, se $F$ e $G$ são dois desdobramentos versais a $r$-parâmetros quaisquer de $f\left(\right.$ em $\left.t_{0}\right)$ e $g$ (em $\left.t_{1}\right)$, respectivamente, ambos do tipo $A_{k}(k \geq 1)$, temos

$$
\begin{aligned}
& F: \mathbb{R} \times \mathbb{R}^{r},\left(t_{0}, x_{0}\right) \rightarrow \mathbb{R} \\
& G: \mathbb{R} \times \mathbb{R}^{r},\left(t_{1}, u_{0}\right) \rightarrow \mathbb{R}
\end{aligned}
$$

onde $F\left(t, x_{0}\right)=f(t)$ ( $t$ próximo a $\left.t_{0}\right), G\left(t, u_{0}\right)=g(t)$ ( $t$ próximo a $\left.t_{1}\right), f$ tendo tipo $A_{k}$ em $t_{0}$ e $g$ tendo tipo $A_{k}$ em $t_{1}$. Então existem vizinhanças de $U$ de $\left(t_{0}, x_{0}\right)$ e $V$ de $\left(t_{1}, u_{0}\right)$ em $\mathbb{R} \times \mathbb{R}^{r}$ tal que $D_{F} \cap U$ e $D_{G} \cap V$ são $r$-variedades parametrizadas.

Proposição 2.28. (Unicidade do conjunto discriminante) Sejam $F$ e $G$ dois desdobramentos versais a r-parâmetros quaisquer de $f$ (em $\left.t_{0}\right)$ e $g$ (em $\left.t_{1}\right)$, respectivamente, ambos do tipo $A_{k}(k \geq 1)$. Então existem vizinhanças $U$ de $t_{0}$ e $V$ de $t_{1}$ tal que existe um difeomorfismo $\phi: U \rightarrow V$ da forma $\phi(t, x)=(a(t, x), b(x))$ onde $a\left(t_{0}, x_{0}\right)=t_{1}, b\left(x_{0}\right)=u_{0}$ $e$ 
1. $\phi\left(M_{F} \cap U\right)=M_{G} \cap V$

2. b é um difeomorfismo de $\pi(U)$ a $\pi(V)$, onde cada $\pi$ é projeção dos parâmetros de desdobramento, e $b\left(\mathcal{D}_{F} \cap \pi(U)\right)=\mathcal{D}_{G} \cap \pi(V)$.

Demonstração: $\operatorname{Ver}([3]$, p. 145).

Esta proposição diz que os conjuntos discriminantes $\mathcal{D}_{F}$ e $\mathcal{D}_{G}$ são localmente difeomorfos.

\subsubsection{Desdobramento versal de $A_{1}(r \geq 1)$}

$F: \mathbb{R} \times \mathbb{R}^{r},(0,0) \rightarrow \mathbb{R},(r \geq 1)$ definido como $F\left(t, x_{1}, \ldots, x_{r}\right)=t^{2}+x_{1}$ é um desdobramento versal de $f: \mathbb{R}, 0 \rightarrow \mathbb{R}$ definido por $f(t)=t^{2}$. O conjunto dos zeros $M_{F}$ é

$$
M_{F}=\left\{\left(t, x_{1}, \ldots, x_{r}\right) \in \mathbb{R} \times \mathbb{R}^{r}: t^{2}+x_{1}=0\right\},
$$

e o conjunto discriminante é

$$
\mathcal{D}_{F}=\left\{\left(x_{1}, \ldots, x_{r}\right) \in \mathbb{R}^{r}: \text { existe } t \in \mathbb{R} t^{2}+x_{1}=2 t=0\right\},
$$

daí $\mathcal{D}_{F}=\{0\} \times \mathbb{R}^{r-1}$.

O mesmo procedimento para o desdobramento $G: \mathbb{R} \times \mathbb{R}^{r},(0,0) \rightarrow \mathbb{R}(r \geq 1)$

$$
G\left(t, x_{1}, \ldots, x_{r}\right)=-t^{2}+x_{1},
$$

da função $g: \mathbb{R}, 0 \rightarrow \mathbb{R}, g(t)=-t^{2}$ nos diz que $\mathcal{D}_{G}=\{0\} \times \mathbb{R}^{r-1}$.

\subsubsection{Desdobramento versal de $A_{2}(r \geq 2)$}

Temos que $F: \mathbb{R} \times \mathbb{R}^{r},(0,0) \rightarrow \mathbb{R},(r \geq 2)$ definido como $F\left(t, x_{1}, \ldots, x_{r}\right)=t^{3}+x_{1}+x_{2} t$ é um desdobramento versal de $f ; \mathbb{R}, 0 \rightarrow \mathbb{R}, f(t)=t^{3}$ então, o conjunto discriminante é

$$
\mathcal{D}_{F}=\left\{\left(x_{1}, \ldots, x_{r}\right) \in \mathbb{R}^{r}: \text { existe } t \in \mathbb{R} \text { com } t^{3}+x_{1}+x_{2} t=3 t^{2}+x_{2}=0\right\},
$$

desenvolvendo as relações obtemos que

$$
\mathcal{D}_{F}=\left\{\left(x_{1}, \ldots, x_{r}\right) \in \mathbb{R}^{r}: 27 x_{1}^{2}+4 x_{2}^{3}=0\right\} .
$$

Assim $\mathcal{D}_{F}$ é difeomorfo a $C \times \mathbb{R}^{r-2}$, onde $C=\left\{\left(x_{1}, x_{2}\right) / x_{1}^{2}=x_{2}^{3}\right\}$. 


\subsection{Conjuntos bifurcação e discriminante}

Podemos resumir o que fizemos em (2.4.2), (2.4.3), (2.5.2) e (2.5.3) da seguinte maneira.

Proposição 2.29. Seja $F: \mathbb{R} \times \mathbb{R}^{r},\left(t_{0}, x_{0}\right) \rightarrow \mathbb{R}$ um desdobramento a r-parâmetros de $f: \mathbb{R}, t_{0} \rightarrow \mathbb{R}$, com $f$ tendo singularidade tipo $A_{k}$ em $t_{0}$.

1. Seja F desdobramento (p)versal,

(a) se $k=2$ então $\mathcal{B}_{F}$ é difeomorfo a $\{0\} \times \mathbb{R}^{r-1}$, e

(b) se $k=3$ então $\mathcal{B}_{F}$ é difeomorfo a $C \times \mathbb{R}^{r-2}$

2. Seja F desdobramento versal,

(a) se $k=1$ então $\mathcal{D}_{F}$ é difeomorfo a $\{0\} \times \mathbb{R}^{r-1}$, e

(b) se $k=2$ então $\mathcal{D}_{F}$ é difeomorfo a $C \times \mathbb{R}^{r-2}$,

onde $C=\left\{\left(x_{1}, x_{2}\right) / x_{1}^{2}=x_{2}^{3}\right\}$. 


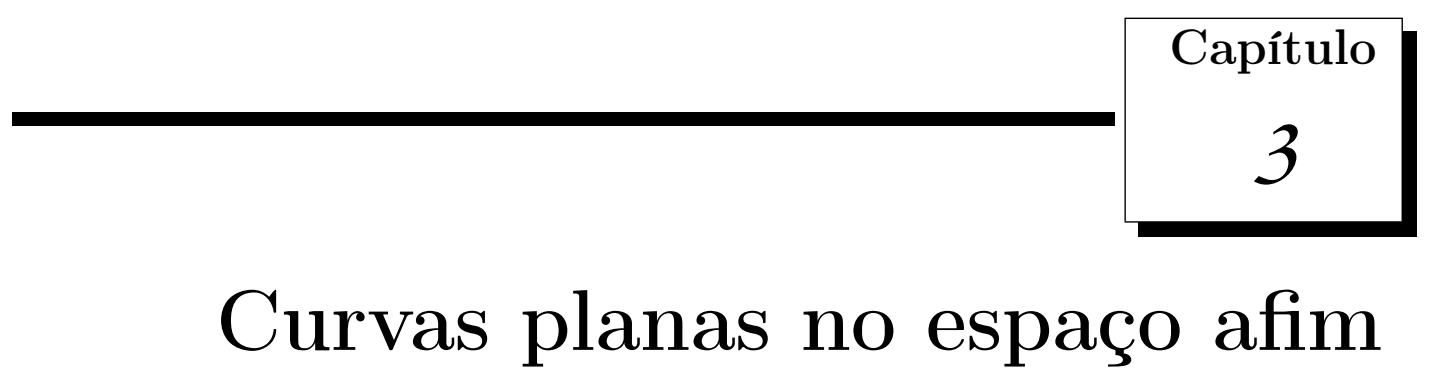

Estamos interessados em estudar a geometria de curvas planas desde o ponto de vista da geometria diferencial afim. Para isto primeiro estudamos as geometrias euclidiana e afim do ponto de vista de Klein, desenvolvido em [2], que estuda propriedades de figuras invariantes pela ação de um grupo, no caso da geometria Euclidiana estuda as propriedades de figuras invariantes por isometrias, no caso da geometria afim estuda as propriedades de figuras invariantes por transformações afins. Na segunda seção estudamos o espaço afim que é o espaço ambiente onde se definem as transformações afins as quais definem a geometria afim. Na terceira seção estudamos conceitos próprios da geometria diferencial afim tais como comprimento de arco afim, curvatura afim e na seção final estudamos as funções suporte afim.

\subsection{Geometria Euclidiana - geometria afim}

O objetivo desta seção é ver as diferenças entre as geometria Euclidiana e geometria afim. Uma geometria consiste de um espaço, algumas propriedades que possuem as figuras neste espaço e um grupo de transformações do espaço que preservam estas propriedades. Este é o chamado "Kleinian view of Geometry". Para o nosso caso, em que estudamos a geometria afim no plano $\mathbb{R}^{2}$, é usado o grupo de transformações da forma $t(x)=A x+a$, onde $a \in \mathbb{R}^{2}$ e $A$ é uma matriz invertível $2 \times 2$. A principal referência nesta seção é o livro de Brannan, Esplen e Gray [2]. 


\subsubsection{Geometria Euclidiana}

Definição 3.1. Uma isometria de $\mathbb{R}^{2}$ é uma função contínua e sobrejetiva $f: \mathbb{R}^{2} \rightarrow \mathbb{R}^{2}$ que preserva distâncias.

Observemos que qualquer isometria tem algumas das seguintes formas ou é composição entre estas.

- Uma translação em $\mathbb{R}^{2}$.

- Uma reflexão com relação a uma reta in $\mathbb{R}^{2}$.

- Uma rotação ao redor de um ponto em $\mathbb{R}^{2}$.

Observemos que o conjunto $\mathcal{S}\left(\mathbb{R}^{2}\right)$ de isometrias forma um grupo com a operação de composição de funções.

Definição 3.2. Uma transformação Euclidiana de $\mathbb{R}^{2}$ é uma função $t: \mathbb{R}^{2} \rightarrow \mathbb{R}^{2} d a$ forma

$$
t(x)=U x+a
$$

onde $U$ é matriz ortogonal $2 \times 2$ e $a \in \mathbb{R}^{2}$.

Uma matriz $U$ é ortogonal se, $U^{-1}=U^{T}$ isto é, $U^{T} U=I$. Equivalentemente uma matriz é ortogonal se, e somente se, as colunas de $U$ são ortonormais.

Teorema 3.3. Qualquer isometria de $\mathbb{R}^{2}$ é uma transformação Euclidiana de $\mathbb{R}^{2}$ e reciprocamente toda transformação Euclidiana é uma isometria.

Demonstração: $\operatorname{Ver}([2]$, p. 49).

Observemos que o conjunto de transformações Euclidianas de $\mathbb{R}^{2}$ forma um grupo com a operação de composição de funções.

Definição 3.4. Uma figura $F_{1}$ é Euclidiana-congruente com uma figura $F_{2}$ se existe uma transformação Euclidiana que transforma $F_{1}$ sobre $F_{2}$.

A relação Euclidiana-congruente é uma relação de equivalência.

Exemplo 3.5. O conjunto de todos os segmentos de reta de comprimento 1 são Euclidianocongruentes.

Exemplo 3.6. Todos os triângulos não são Euclidiano-congruentes.

Em resumo geometria Euclidiana é o estudo das propriedades de figuras invariantes pelo grupo de isometrias. Chamamos estas propriedades de propriedades Euclidianas. 


\subsubsection{Geometria afim}

Entendemos geometria afim como o estudo das propriedades de figuras invariantes pelo grupo de transformações afins.

Definição 3.7. Uma transformação afim de $\mathbb{R}^{2}$ é uma função $t: \mathbb{R}^{2} \rightarrow \mathbb{R}^{2}$ da forma

$$
t(x)=A x+b,
$$

onde $A$ é uma matriz invertivel $2 \times 2$ e $b \in \mathbb{R}^{2}$. O conjunto de transformações afins de $\mathbb{R}^{2}$ é denotado por $A(2)$.

Teorema 3.8. O conjunto de transformações afins A(2) forma um grupo com a operação de composição de funções.

Demonstração: Sejam $t_{1}, t_{2}: \mathbb{R}^{2} \rightarrow \mathbb{R}^{2}$ duas transformações afins, dadas por $t_{1}(x)=$ $A_{1} x+b_{1}$ e $t_{2}(x)=A_{2} x+b_{2}, \operatorname{logo} t_{1} \circ t_{2}(x)=A_{1} A_{2} x+\left(A_{1} b_{2}+b_{1}\right)$, como $A_{1} A_{2}$ é invertível, esta é transformação afim. A inversa de uma transformação afim $t(x)=A x+b$ é dada por $t^{-1}(x)=A^{-1} x-A^{-1} b$.

Observemos que as transformações Euclideanas são transformações afins.

\section{Propriedades básicas de transformações afins:}

Transformações afins,

1. levam retas em retas,

2. levam retas paralelas em retas paralelas,

3. preservam razão de comprimentos ao longo de uma reta dada.

Teorema 3.9. (Teorema fundamental da geometria afim) Sejam $p, q, r$ e $p^{\prime}, q^{\prime}, r^{\prime}$ dois conjuntos de pontos não colineares em $\mathbb{R}^{2}$. Então;

1. existe uma transformação afim $t$ que satisfaz

$$
t(p)=p^{\prime}, t(q)=q^{\prime} \text { e } t(r)=r^{\prime}
$$

2. a transformação afim t é única.

Demonstração: $\operatorname{Ver}([2]$, p. 69). 
Definição 3.10. Uma figura $F_{1}$ é afim-congruente com uma figura $F_{2}$ se existe uma transformação afim que transforma $F_{1} \mathrm{em} F_{2}$.

A relação afim-congruente é uma relação de equivalência.

Corolário 3.11. Todos os triângulos são afim-congruentes.

Demonstração: Segue do Teorema (3.9) e de que as transformações afins levam retas em retas.

Agora a questão é: "Quais outras figuras planas são afim congruentes?".

\subsubsection{Transformações afins e cônicas}

Definição 3.12. Uma cônica é um conjunto em $\mathbb{R}^{2}$ definido pela equação da forma

$$
A x^{2}+B x y+C y^{2}+F x+G y+H=0,
$$

onde $A, B, C, F, G$ e $H$ são números reais, e $A, B$ e $C$ não todos nulos.

As três formas de cônica não degenerada são elipses, parábolas e hipérboles. Uma cônica não degenerada é uma:

- hipérbole se $B^{2}-4 A C>0$,

- parábola se $B^{2}-4 A C=0$,

- elipse se $B^{2}-4 A C<0$.

O número $\Delta=B^{2}-4 A C$ é chamado de discriminante da cônica.

O próximo resultado mostra a grande diferença entre as geometrias afim e Euclidiana.

Teorema 3.13. Qualquer elipse é afim-congruente ao círculo unitário $x^{2}+y^{2}=1$.

Demonstração: Qualquer elipse é afim-congruente com a elipse na forma $\frac{x^{2}}{a^{2}}+\frac{y^{2}}{b^{2}}=1$, pois fazendo uma rotação e translação obtemos tal forma.

Agora consideremos a transformação afim $t_{1}:(x, y) \mapsto\left(x^{\prime}, y^{\prime}\right)$ onde,

$$
\left(\begin{array}{l}
x^{\prime} \\
y^{\prime}
\end{array}\right)=\left(\begin{array}{cc}
\frac{1}{a} & 0 \\
0 & \frac{1}{b}
\end{array}\right)\left(\begin{array}{l}
x \\
y
\end{array}\right)
$$

daí, $\left(x^{\prime}\right)^{2}+\left(y^{\prime}\right)^{2}=1$. 
Teorema 3.14. Qualquer hipérbole é afim-congruente à hipérbole retangular $x y=1$.

Demonstração: Qualquer hipérbole é afim-congruente com a hipérbole da forma $\frac{x^{2}}{a^{2}}-\frac{y^{2}}{b^{2}}=1$, pois por rotação e translação obtemos tal forma.

Agora consideremos a transformação afim $t_{1}:(x, y) \mapsto\left(x^{\prime}, y^{\prime}\right)$, onde

$$
\left(\begin{array}{l}
x^{\prime} \\
y^{\prime}
\end{array}\right)=\left(\begin{array}{cc}
\frac{1}{a} & 0 \\
0 & \frac{1}{b}
\end{array}\right)\left(\begin{array}{l}
x \\
y
\end{array}\right)
$$

$\operatorname{logo}\left(x^{\prime}\right)^{2}-\left(y^{\prime}\right)^{2}=1$ daí $\left(x^{\prime}-y^{\prime}\right)\left(x^{\prime}+y^{\prime}\right)=1$.

Finalmente, aplicando a transformação afim $t_{2}:\left(x^{\prime}, y^{\prime}\right) \mapsto\left(x^{\prime \prime}, y^{\prime \prime}\right)$, onde

$$
\left(\begin{array}{l}
x^{\prime \prime} \\
y^{\prime \prime}
\end{array}\right)=\left(\begin{array}{cc}
1 & -1 \\
1 & 1
\end{array}\right)\left(\begin{array}{l}
x^{\prime} \\
y^{\prime}
\end{array}\right)
$$

obtemos $x^{\prime \prime} y^{\prime \prime}=1$.

Teorema 3.15. Qualquer parábola é afim-congruente à parábola $y^{2}=x$.

Demonstração: Qualquer parábola é afim-congruente com a parábola $y^{2}=a x$. Por rotação e translação obtemos tal forma. Agora consideremos a transformação afim $t$ : $(x, y) \mapsto\left(x^{\prime}, y^{\prime}\right)$, onde

$$
\left(\begin{array}{l}
x^{\prime} \\
y^{\prime}
\end{array}\right)=\left(\begin{array}{cc}
\frac{1}{a} & 0 \\
0 & \frac{1}{a}
\end{array}\right)\left(\begin{array}{l}
x \\
y
\end{array}\right)
$$

com isto $\left(y^{\prime}\right)^{2}=x^{\prime}$

\subsection{O espaço afim}

Nesta seção definimos espaço afim, subespaço afim e sistema de coordenadas afim. As definições e resultados desta seção são encontrados na referência [1].

Definição 3.16. Seja $V$ um espaço vetorial real n-dimensional. Um conjunto não vazio $\Omega$ é chamado um espaço afim associado com $V$ se, existe uma função $\pi: \Omega \times \Omega \rightarrow V$ que associa para cada par $(p, q)$ um vetor em $V$ denotado por $\overrightarrow{p q}$, que satisfaz os seguintes axiomas:

1. $\operatorname{para} p, q, r \in \Omega$ temos $\overrightarrow{p r}=\overrightarrow{p q}+\overrightarrow{q r}$, 
2. para qualquer $p \in \Omega$ e qualquer $x \in V$, existe um único $q \in \Omega$ tal que $\overrightarrow{p q}=x$.

Os elementos de $\Omega$ são chamados pontos. A dimensão de $\Omega$ é definida como a dimensão do espaço vetorial associado $V$.

Exemplo 3.17. Todo espaço vetorial real de dimensão finita é um espaço afim.

Exemplo 3.18. O produto cartesiano finito de espaços afins é um espaço afim.

Fazendo um cálculo simples e usando o Axioma 1 de espaço afim tem-se:

1. $\overrightarrow{p p}=0, \quad \forall p \in \Omega$

2. $\overrightarrow{q p}=-\overrightarrow{p q}, \quad \forall p, q \in \Omega$.

Observação 3.19. Dado $p \in \Omega$ fixado, para qualquer $x \in V$, existe um único $q \in \Omega$ tal que $\overrightarrow{p q}=x$, as vezes é conveniente escrever $q=p+x$ para representar $x=\overrightarrow{p q}$, esta notação é consistente pois se satisfaz, $p+(x+y)=(p+x)+y$.

Seja $\Omega$ um espaço afim associado com o espaço vetorial $V$. Se escolhemos um ponto $\mathrm{O}$ em $\Omega$, podemos dar uma estrutura de espaço vetorial a $\Omega$. De fato, basta definir:

$$
\begin{gathered}
p+q \triangleq \pi^{-1}(\overrightarrow{\mathbf{O} p}+\overrightarrow{\mathbf{O} q}) \\
\lambda p \triangleq \pi^{-1}(\lambda \overrightarrow{\mathbf{O} p})
\end{gathered}
$$

Definição 3.20. Um sistema de coordenadas afim com origem $\boldsymbol{O} \in \Omega$ é definido como segue: Fixamos $\left\{e_{1}, \ldots, e_{n}\right\}$ uma base de $V$, logo para qualquer ponto $p \in \Omega, \overrightarrow{O p} \in V$ e então existe uma $n$-upla $\left(x^{1}(p), \ldots, x^{n}(p)\right)$ de números tal que

$$
\overrightarrow{O p}=\sum_{i=1}^{n} x^{i}(p) e_{i}
$$

Uma $n$-upla $\left(x^{1}(p), \ldots, x^{n}(p)\right)$ assim definida é chamada de coordenadas do ponto $p$. O conjunto de funções $\left\{x^{1}, \ldots, x^{n}\right\}$ é chamado um sistema de coordenadas afim.

Dois sistemas de coordenadas afins $\left\{x^{1}, \ldots, x^{n}\right\}$ e $\left\{y^{1}, \ldots, y^{n}\right\}$ estão relacionados da forma seguinte:

$$
y^{j}=\sum_{i=1}^{n} a_{j}^{i} x^{j}+c^{i} \quad 1 \leq i \leq n,
$$

onde $A=\left[a_{j}^{i}\right]$ é uma matriz não singular e $C=\left[c^{i}\right]$ é um vetor. 
Agora seja $\Omega$ um espaço afim associado com o espaço vetorial $V$ e seja $f: \Omega \rightarrow \Omega$ uma função injetora de $\Omega$ sobre si mesmo. Para qualquer $p \in \Omega$ definimos a função

$$
\begin{aligned}
& F_{p}: V \rightarrow V \\
& x=\overrightarrow{p r} \mapsto F_{p}(x)=\overrightarrow{f(p) f(r) .}
\end{aligned}
$$

Definição 3.21. Dizemos que $f: \Omega \rightarrow \Omega$ é uma transformação afim se, para algum $p \in \Omega$, a função $F_{p}: V \rightarrow V$ é uma transformação linear de $V$ sobre si mesmo.

Teorema 3.22. Seja $\Omega$ um espaço afim associado com o espaço vetorial $V$ e $f: \Omega \rightarrow \Omega$ uma transformação afim. Então $F_{q}=F_{r}, \forall q, r \in \Omega$.

Demonstração: Como $f$ é uma transformação afim, $\exists p \in \Omega$ tal que $F_{p}$ é linear. Por outro lado, para $x \in V$ existe um único $s \in \Omega$ tal que $x=\overrightarrow{q s}$, logo

$$
F_{q}(x)=\overrightarrow{f(q) f(s)}=\overrightarrow{f(q) f(p)}+\overrightarrow{f(p) f(s)},
$$

daí $F_{q}(x)=-\overrightarrow{f(p) f(q)}+\overrightarrow{f(p) f(s)}=-F_{p}(\overrightarrow{p q})+F_{p}(\overrightarrow{p s})=F_{p}(\overrightarrow{p s}-\overrightarrow{p q})=F_{p}(\overrightarrow{q s})$, e assim

$$
F_{q}(x)=F_{p}(\overrightarrow{q s})=F_{p}(x), \forall x \in V
$$

O teorema acima diz que a transformação afim não depende do ponto $p$, logo podemos escrever $F$ em vez de $F_{p}$.

Na seção anterior definimos transformação afim de $\mathbb{R}^{2}$ como uma função $t: \mathbb{R}^{2} \rightarrow \mathbb{R}^{2}$ da forma

$$
t(x)=A x+b
$$

onde $A$ é uma matriz invertível $2 \times 2$ e $b \in \mathbb{R}^{2}$. Esta noção coincide com a noção recentemente definida no caso que $V=\mathbb{R}^{2}$.

De fato, seja $A=\left(\begin{array}{ll}a_{1,1} & a_{1,2} \\ a_{2,1} & a_{2,2}\end{array}\right)$ e $b=\left(\begin{array}{l}b_{1} \\ b_{2}\end{array}\right), \operatorname{logo} t: \mathbb{R}^{2} \rightarrow \mathbb{R}^{2}$ tem a seguinte forma

$$
t(x, y)=\left(a_{1,1} x+a_{1,2} y+b_{1}, a_{2,1} x+a_{2,2} y+b_{2}\right)
$$

Consideremos $p=\left(b_{1}, b_{2}\right)$ e $(x, y)=\overrightarrow{\left(b_{1}, b_{2}\right)\left(x^{\prime}, y^{\prime}\right)}$. Igualando coordenada a coordenada obtemos a seguintes relações, $x=x^{\prime}-b_{1}$ e $y=y^{\prime}-b_{2}$. Por outro lado, $F_{p}(x, y)=$ $\overrightarrow{t\left(b_{1}, b_{2}\right) t\left(x^{\prime}, y^{\prime}\right)}$ e tem-se $F_{p}(x, y)=\left(a_{1,1}\left(x^{\prime}-b_{1}\right)+a_{1,2}\left(y^{\prime}-b_{2}\right), a_{2,1}\left(x^{\prime}-b_{1}\right)+a_{2,2}\left(y^{\prime}-b_{2}\right)\right)$. Daí $F_{p}(x, y)=t(x, y)$. 
Se $\left\{x^{1}, \ldots, x^{n}\right\}$ é um sistema de coordenadas afim com origem $\mathbf{O}$ sobre uma base $\left\{e_{1}, \ldots, e_{n}\right\}$, e $\left\{y^{1}, \ldots, y^{n}\right\}$ um outro sistema de coordenadas afim com origem $f(\mathbf{O})$ sobre a base $\left\{F\left(e_{1}\right), \ldots, F\left(e_{n}\right)\right\}$ então $y^{i}(f(p))=x^{i}(p), \forall p \in \Omega$. Podemos escrever a relação entre os sistemas coordenados $\left\{x^{1}, \ldots, x^{n}\right\}$ e $\left\{y^{1}, \ldots, y^{n}\right\}$ na forma

$$
x^{j}=\sum_{i=1}^{n} b_{j}^{i} y^{j}+d^{i} \quad 1 \leq i \leq n,
$$

além disso, relativo ao sistema de coordenadas $\left\{x^{1}, \ldots, x^{n}\right\}$ as coordenadas de $\bar{x}^{i}$ são

$$
\bar{x}^{i}=x^{i}(f(p))=\sum_{i=1}^{n} b_{j}^{i} y^{j}(f(p))+d^{i}=\sum_{i=1}^{n} b_{j}^{i} x^{j}(p)+d^{i} .
$$

Definição 3.23. Um subconjunto não vazio $\Omega^{\prime}$ de $\Omega$ é chamado subespaço afim se existe um ponto $p \in \Omega^{\prime}$ tal que o conjunto de vetores $W_{p}=\left\{\overrightarrow{p q}: q \in \Omega^{\prime}\right\}$ forma um subespaço vetorial de $V$.

Proposição 3.24. Seja $\Omega^{\prime}$ um subespaço afim de $\Omega$. Então o espaço vetorial $W_{p}$ independe da escolha de $p \in \Omega$.

Demonstração: Seja $r \in \Omega^{\prime}$, temos que provar que $W_{p}=W_{r}$.

De fato, seja $x \in W_{r}$ então existe $q \in \Omega^{\prime}$ tal que

$$
x=\overrightarrow{r q}=\overrightarrow{r p}+\overrightarrow{p q}=-\overrightarrow{p r}+\overrightarrow{p q} \in W_{p}
$$

portanto $W_{r} \subseteq W_{p}$.

Agora a outra inclusão. Seja $y \in W_{p}$, então existe $q \in \Omega^{\prime}$ tal que $y=\overrightarrow{p q}$. Por outro lado existe $s \in \Omega$ tal que $y=\overrightarrow{r s}$. Logo $\overrightarrow{p q}=\overrightarrow{r s}$ e daí

$$
\overrightarrow{p s}=\overrightarrow{p r}+\overrightarrow{r s}=\overrightarrow{p r}+\overrightarrow{r s}=\overrightarrow{p r}+\overrightarrow{p q} \in W_{p}
$$

Assim $\overrightarrow{p s} \in W_{p}$ e portanto $s \in \Omega^{\prime}$ e $y=\overrightarrow{r s}$. Logo $y \in W_{r}$ de onde conclui-se que $W_{p} \subseteq W_{r}$.

\subsection{Comprimento de arco afim - curvatura afim}

Nesta seção, toda curva e aplicações serão consideradas suaves (de classe $C^{\infty}$ ). Identificamos o espaço Euclidiano $\mathbb{R}^{2}$ com o espaço das matrizes reais $2 \times 1, M_{2 \times 1}(\mathbb{R})$ da forma 
usual

$$
(a, b) \leftrightarrow\left[\begin{array}{l}
a \\
b
\end{array}\right]
$$

Com isto, se $a=\left(a_{1}, a_{2}\right)$ e $b=\left(b_{1}, b_{2}\right)$ então $\left[\begin{array}{ll}a & b\end{array}\right]$ denota a matriz $\left[\begin{array}{ll}a_{1} & b_{1} \\ a_{2} & b_{2}\end{array}\right]$ e com $|a, b|$ denotamos o determinante da matriz $\left[\begin{array}{ll}a & b\end{array}\right]$, ou seja

$$
|a, b|=a_{1} b_{2}-a_{2} b_{1}
$$

\subsubsection{Comprimento de arco afim}

Seja $\gamma: I \rightarrow \mathbb{R}^{2}$ uma curva plana, $\gamma(t)=(x(t), y(t)), \forall t \in I$, onde $x: I \rightarrow \mathbb{R} \mathrm{e}$ $y: I \rightarrow \mathbb{R}$ são funções suaves de $t$.

Definição 3.25. Um plano afim é o espaço afim $\mathbb{R}^{2}$ associado com o espaço vetorial Euclidiano $\mathbb{R}^{2}$ da forma usual, considerando a função $\pi: \mathbb{R}^{2} \times \mathbb{R}^{2} \rightarrow \mathbb{R}^{2}$, que associa cada par $(p, q)$ o vetor $q-p$ em $\mathbb{R}^{2}$.

Observação 3.26. Sejam $a=\left(a_{1}, a_{2}\right)$ e $b=\left(b_{1}, b_{2}\right)$ dois vetores linearmente independentes em $\mathbb{R}^{2}$, fixando $\boldsymbol{O}=(p, q) \in \mathbb{R}^{2}$, para qualquer $P=(\bar{x}, \bar{y}) \in \mathbb{R}^{2}$, existem $x, y \in \mathbb{R}$ tal que

$$
\overrightarrow{O P}=a x+b y
$$

$(x, y)$ são as coordenadas afim de P (ver Definição (3.20)).

Desenvolvendo a relação acima obtemos

$$
\begin{aligned}
& \bar{x}=a_{1} x+b_{1} y+p \\
& \bar{y}=a_{2} x+b_{2} y+q .
\end{aligned}
$$

Desde que $a=\left(a_{1}, a_{2}\right)$ e $\left(b_{1}, b_{2}\right)$ são dois vetores linearmente independentes em $\mathbb{R}^{2}$ o determinante da matriz $\left[\begin{array}{ll}a & b\end{array}\right]$ é distinto de zero, ou seja $a_{1} b_{2}-a_{2} b_{1} \neq 0$.

Observação 3.27. O determinante dos vetores linearmente independentes $a=\left(a_{1}, a_{2}\right)$ e $\left(b_{1}, b_{2}\right)$ representa o área do paralelogramo gerado por estes vetores.

De acordo com a observação (3.26) um plano afim $\mathbb{R}^{2}$ é referido em termos de um sistema de coordenadas $\{x, y\}$, como acima o qual considera as coordenadas tal que o área do paralelogramo gerado por dois vetores $a=\left(a_{1}, a_{2}\right)$ e $b=\left(b_{1}, b_{2}\right)$ linearmente independentes é dado por $|a, b|=a_{1} b_{2}-a_{2} b_{1}$. 
Observemos que se $\alpha: I \rightarrow \mathbb{R}^{2}$ e $\beta: I \rightarrow \mathbb{R}^{2}$ são duas curvas planas suaves então a derivada da função $g: I \rightarrow \mathbb{R}^{2}$, definida por $g(t)=|\alpha(t), \beta(t)|$ é dada pela expressão

$$
\dot{g}(t)=|\dot{\alpha}(t), \beta(t)|+|\alpha(t), \dot{\beta}(t)|
$$

Definição 3.28. Uma curva $\gamma: I \rightarrow \mathbb{R}^{2}$ é não degenerada se satisfaz $|\dot{\gamma}(t), \ddot{\gamma}(t)| \neq 0$, $\forall t \in I$.

Seja $\gamma: I \rightarrow \mathbb{R}^{2}$ uma curva não degenerada, $\gamma(t)=(x(t), y(t)), \forall t \in I$. Denotemos por $a_{1}(t)=\dot{\gamma}(t)$ e consideremos $a_{2}(t)$ outro vetor que satisfaz $\left|a_{1}(t), a_{2}(t)\right|=1, \forall t \in I$, isto é $\left[a_{1}(t) \quad a_{2}(t)\right] \in S L(2, \mathbb{R})$. Construimos um novo parâmetro (no sentido da Definição (1.4)) $\sigma: I \rightarrow J$ para a curva $\gamma$ tal que

$$
\left[\gamma^{\prime}(s) \gamma^{\prime \prime}(s)\right] \in S L(2, \mathbb{R}), \forall s \in J
$$

onde o símbolo' denota a derivada com relação ao novo parâmetro $s=\sigma(t)$.

Agora vamos fixar notações, se $\gamma: I \rightarrow \mathbb{R}^{2}$ é uma curva não degenerada e $h: I \rightarrow J$ um difeomorfismo, $\gamma \circ \sigma^{-1}: J \rightarrow \mathbb{R}^{2}$ é uma reparametrização da curva $\gamma$.

Usamos as seguintes notações:

$$
\dot{\gamma}(t)=\frac{d \gamma}{d t}(t), \forall t \in I
$$

para a derivada de $\gamma: I \rightarrow \mathbb{R}^{2}$, e a notação

$$
\gamma^{\prime}(s)=\frac{d\left(\gamma \circ \sigma^{-1}\right)}{d s}(s), \forall s \in J
$$

para a derivada da outra parametrização $\gamma \circ \sigma^{-1}: J \rightarrow \mathbb{R}^{2}$.

Teorema 3.29. Se $\gamma: I \rightarrow \mathbb{R}^{2}$ é uma curva não degenerada, então existe $\sigma: I \rightarrow J$ difeomorfismo tal que $\left[\gamma^{\prime}(s) \gamma^{\prime \prime}(s)\right] \in S L(2, \mathbb{R}), \forall s \in J$.

Demonstração: Suponhamos que existe difeomorfismo $\sigma: I \rightarrow J$ tal que satisfaz $\left[\gamma^{\prime}(s) \gamma^{\prime \prime}(s)\right] \in S L(2, \mathbb{R}), \forall s \in J$. Agora consideremos a reparametrizaçaõ $\alpha_{1}: J \rightarrow \mathbb{R}^{2}$ definida por $\alpha_{1}(s)=\gamma \circ \sigma^{-1}(s), \forall s \in J$. Assim $\gamma(t)=\alpha_{1}(\sigma(t))$. Por diferenciação $\dot{\gamma}(t)=\alpha_{1}^{\prime}(s) \dot{\sigma}(t)$ e $\ddot{\gamma}(t)=\alpha_{1}^{\prime \prime}(s) \dot{\sigma}(t)^{2}+\alpha_{1}^{\prime}(s) \ddot{\sigma}(t)$. Logo

$$
\begin{aligned}
|\dot{\gamma}(t), \ddot{\gamma}(t)|= & \left|\alpha_{1}^{\prime}(s) \dot{\sigma}(t), \alpha_{1}^{\prime \prime}(s) \dot{\sigma}(t)^{2}+\alpha_{1}^{\prime}(s) \ddot{\sigma}(t)\right| \\
& =\left|\alpha_{1}^{\prime}(s) \dot{\sigma}(t), \alpha_{1}^{\prime \prime}(s) \dot{\sigma}(t)^{2}\right|+\left|\alpha_{1}^{\prime}(s) \dot{\sigma}(t), \alpha_{1}^{\prime}(s) \ddot{\sigma}(t)\right| \\
& =\left|\alpha_{1}^{\prime}(s) \dot{\sigma}(t), \alpha_{1}^{\prime \prime}(s) \dot{\sigma}(t)^{2}\right|
\end{aligned}
$$




$$
=\dot{\sigma}(t)^{3}\left|\alpha_{1}^{\prime}(s), \alpha_{1}^{\prime \prime}(s)\right|
$$

Por outro lado $\left[\alpha_{1}^{\prime}(s) \alpha_{1}^{\prime \prime}(s)\right] \in S L(2, \mathbb{R}), \forall s \in J$. Então $\left.\mid \alpha_{1}^{\prime}(s), \alpha_{1}^{\prime \prime}(s)\right]=1$ e, portanto, $|\dot{\gamma}(t), \ddot{\gamma}(t)|=\dot{\sigma}(t)^{3}$. Assim tem-se,

$$
\frac{d \sigma}{d t}=|\dot{\gamma}(t), \ddot{\gamma}(t)|^{\frac{1}{3}}
$$

Fixando um ponto $t_{0} \in I$ definimos

$$
\sigma(t)=\int_{t_{0}}^{t}|\dot{\gamma}(x), \ddot{\gamma}(x)|^{\frac{1}{3}} d x
$$

Usando a Definição (1.4), como $\sigma$ é suave e $\sigma^{\prime}(t) \neq 0, \forall t \in I$, então $\sigma$ é um difeomorfismo.

Definição 3.30. O comprimento de arco afim de uma curva $\gamma: I \rightarrow \mathbb{R}^{2}$ medido a partir de $\gamma\left(t_{0}\right), t_{0} \in I$, é definida como

$$
\sigma(t)=\int_{t_{0}}^{t}|\dot{\gamma}(u), \ddot{\gamma}(u)|^{\frac{1}{3}} d u
$$

onde $\left|\gamma^{\prime}(s), \gamma^{\prime \prime}(s)\right|=1, \forall s=\sigma(t)$.

Dizemos que uma curva $\gamma: I \rightarrow \mathbb{R}^{2}$ esta parametrizada pelo comprimento de arco afim se satisfaz, $\left|\gamma^{\prime}(s), \gamma^{\prime \prime}(s)\right|=1, \forall s \in J=\sigma(I)$.

Fixando $t_{0} \in I$, dizemos que $\sigma$ é uma função comprimento de arco afim.

O Teorema (3.29) diz que, se $\gamma: I \rightarrow \mathbb{R}^{2}$ é uma curva não degenerada então existe uma mudança de parâmetro $\xi: J \rightarrow I$ tal que a curva $\gamma \circ \xi$ está parametrizada por comprimento de arco afim. Uma outra forma de dizer isto é que toda curva não degenerada tem uma reparametrização pelo comprimento de arco afim.

Corolário 3.31. Seja $\gamma: I \rightarrow \mathbb{R}^{2}$ uma curva não degenerada parametrizada por comprimento de arco, então existe um difeomorfismo $\xi: J \rightarrow I$ tal que $\gamma \circ \xi$ esta parametrizada por comprimento de arco afim.

Observação 3.32. As curvas não degeneradas, tem interpretação geométrica.

Para ver a ideia geométrica desta condição suponhamos que $|\dot{\gamma}(t), \ddot{\gamma}(t)|>0, \quad \forall t \in I$. 
Logo pelo Teorema de Taylor, para $h$ suficientemente pequeno

$$
\gamma(t+h)-\gamma(t)=h \dot{\gamma}(t)+\frac{1}{2} h^{2} \ddot{\gamma}(\tau)
$$

onde $\tau$ esta entre $t$ e $t+h$. Assim $|\dot{\gamma}(t), \gamma(t+h)-\gamma(t)|=\left|\dot{\gamma}(t), h \dot{\gamma}(t)+\frac{1}{2} h^{2} \ddot{\gamma}(\tau)\right|$ então $|\dot{\gamma}(t), \gamma(t+h)-\gamma(t)|=\frac{1}{2} h^{2}|\dot{\gamma}(t), \ddot{\gamma}(\tau)|$. Segue da continuidade da função determinante que $|\dot{\gamma}(t), \ddot{\gamma}(\tau)|>0$ e, portanto, tem-se $|\dot{\gamma}(t), \gamma(t+h)-\gamma(t)|>0$.

Concluimos que a secante desde $\gamma(t)$ para $\gamma(t+h)$ pertencem a um mesmo lado da reta tangente. Isto é, a curva $\gamma$ não têm pontos de inflexão. Com isto, dizer que a curva $\gamma$ é não degenerada significa que $\gamma$ não tem inflexões.

Em relação a curvas não degeneradas, em [11] estas são chamadas curvas que não tem pontos de inflexão.

\subsubsection{Curvatura afim}

Definição 3.33. Seja $\gamma: I \rightarrow \mathbb{R}^{2}$ uma curva não degenerada parametrizada por comprimento de arco afim, a curvatura afim de $\gamma$ é a função real $k_{a}: I \rightarrow \mathbb{R}$ definida por $k_{a}(s)=\left|\gamma^{\prime \prime}(s), \gamma^{\prime \prime \prime}(s)\right|$.

Teorema 3.34. Seja $\gamma: I \rightarrow \mathbb{R}^{2}$ uma curva não degenerada parametrizada por comprimento de arco afim. Então

$$
\gamma^{\prime \prime \prime}(s)=-k_{a}(s) \gamma^{\prime}(s)
$$

Demonstração: Como $\gamma$ está parametrizada pelo comprimento de arco afim, então temos $\left|\gamma^{\prime}(s), \gamma^{\prime \prime}(s)\right|=1$, Agora pela relação $(3.2)\left|\gamma^{\prime \prime}(s), \gamma^{\prime \prime}(s)\right|+\left|\gamma^{\prime}(s), \gamma^{\prime \prime \prime}(s)\right|=0$. Como $\left|\gamma^{\prime \prime}(s), \gamma^{\prime \prime}(s)\right|=0$ então $\left|\gamma^{\prime}(s), \gamma^{\prime \prime \prime}(s)\right|=0$. Agora, por propriedade do determinante,

$$
\exists \lambda(s) \in \mathbb{R} \text {, tal que } \gamma^{\prime \prime \prime}(s)=\lambda(s) \gamma^{\prime}(s)
$$

Por outro lado,

$$
\left|\gamma^{\prime \prime}(s), \gamma^{\prime \prime \prime}(s)\right|=\left|\gamma^{\prime \prime}(s), \lambda(s) \gamma^{\prime}(s)\right|=-\lambda(s)\left|\gamma^{\prime}(s), \gamma^{\prime \prime}(s)\right|=-\lambda(s)
$$

$\operatorname{assim} \lambda(s)=-k_{a}(s)$

Observação 3.35. Se $\gamma: I \rightarrow \mathbb{R}^{2}$ é uma curva parametrizada pelo comprimento de arco afim, então a curvatura Euclidiana é sempre positiva. 
Com efeito, a curvatura Euclidiana de uma curva plana é dada pela relação

$$
k(s)=\frac{\left|\gamma^{\prime}(s), \gamma^{\prime \prime}(s)\right|}{\left\|\gamma^{\prime}(s)\right\|^{3}} .
$$

Como $\gamma$ esta parametrizada por comprimento de arco afim então $\left|\gamma^{\prime}(s), \gamma^{\prime \prime}(s)\right|=1$ e portanto $k(s)=\frac{1}{\left\|\gamma^{\prime}(s)\right\|^{3}}>0$.

Exemplo 3.36. A curvatura afim da elipse $\frac{x^{2}}{a^{2}}+\frac{y^{2}}{b^{2}}=1$ é dada pela seguinte equação

$$
k_{a}(s)=(a b)^{-\frac{2}{3}}
$$

De fato, consideremos a parametrização $\alpha(t)=(a \cos t, b \operatorname{sen} t)$. Agora por diferenciação

$$
\dot{\alpha}(t)=(-a \operatorname{sen} t, b \cos t) \text { e } \ddot{\alpha}(t)=(-a \cos t,-b \operatorname{sen} t),
$$

daí $|\dot{\alpha}(t), \ddot{\alpha}(t)|=a b$.

Considerando $t_{0}=0$,

$$
\sigma(t)=\int_{0}^{t}|\dot{\alpha}(u), \ddot{\alpha}(u)|^{\frac{1}{3}} d u=\int_{0}^{t}(a b)^{\frac{1}{3}} d u
$$

Logo $\sigma(t)=(a b)^{\frac{1}{3}}(t)$ é um difeomorfismo com inversa $\sigma^{-1}(s)=(a b)^{-\frac{1}{3}} s$. Por outro lado defina

$$
\gamma(s)=\alpha\left(\sigma^{-1}(s)\right)=\left(a \cos \frac{t}{\sqrt[3]{a b}}, b \operatorname{sen} \frac{t}{\sqrt[3]{a b}}\right)
$$

Temos:

$\gamma^{\prime}(s)=\left(-\frac{a}{\sqrt[3]{a b}} \operatorname{sen} \frac{t}{\sqrt[3]{a b}}, \frac{b}{\sqrt[3]{a b}} \cos \frac{t}{\sqrt[3]{a b}}\right)$,

$\gamma^{\prime \prime}(s)=\left(-\frac{a}{\sqrt[3]{a b}^{2}} \cos \frac{t}{\sqrt[3]{a b}},-\frac{b}{\sqrt[3]{a b}^{2}} \operatorname{sen} \frac{t}{\sqrt[3]{a b}}\right)$,

$\gamma^{\prime \prime \prime}(s)=\left(\frac{1}{b} \operatorname{sen} \frac{t}{\sqrt[3]{a b}},-\frac{1}{a} \cos \frac{t}{\sqrt[3]{a b}}\right)$.

Logo,

$$
k_{a}(s)=\left|\gamma^{\prime \prime}(s), \gamma^{\prime \prime \prime}(s)\right|=(a b)^{-\frac{2}{3}}
$$

Exemplo 3.37. A curvatura afim da hipérbole $\frac{x^{2}}{a^{2}}-\frac{y^{2}}{b^{2}}=1$ é

$$
k_{a}(s)=-(a b)^{-\frac{2}{3}}
$$

De fato, consideremos a parametrização $\alpha(t)=(a \cosh t, b \operatorname{senh} t)$. Agora por diferen- 
ciação

$$
\dot{\alpha}(t)=(a \operatorname{senh} t, b \cosh t) \text { e } \ddot{\alpha}(t)=(a \cosh t, b \operatorname{senh} t),
$$

$\operatorname{logo}|\dot{\alpha}(t), \ddot{\alpha}(t)|=-a b$. Considerando $t_{0}=0$,

$$
\sigma(t)=\int_{0}^{t}|\dot{\alpha}(u), \ddot{\alpha}(u)|^{\frac{1}{3}} d u=\int_{0}^{t}-(a b)^{\frac{1}{3}} d u
$$

então $\sigma(t)=-(a b)^{\frac{1}{3}}(t)$ é um difeomorfismo com inversa $\sigma^{-1}(s)=-(a b)^{-\frac{1}{3}} s$. Por outro lado defina

$$
\gamma(s)=\alpha\left(\sigma^{-1}(s)\right)=\left(a \cosh \frac{t}{\sqrt[3]{a b}},-b \operatorname{senh} \frac{t}{\sqrt[3]{a b}}\right) .
$$

Temos:

$\gamma^{\prime}(s)=\left(\frac{a}{\sqrt[3]{a b}} \operatorname{senh} \frac{t}{\sqrt[3]{a b}},-\frac{b}{\sqrt[3]{a b}} \cosh \frac{t}{\sqrt[3]{a b}}\right)$,

$\gamma^{\prime \prime}(s)=\left(\frac{a}{\sqrt[3]{a b}^{2}} \cosh \frac{t}{\sqrt[3]{a b}},-\frac{b}{\sqrt[3]{a b}^{2}} \operatorname{senh} \frac{t}{\sqrt[3]{a b}}\right)$,

$\gamma^{\prime \prime \prime}(s)=\left(\frac{1}{b} \operatorname{senh} \frac{t}{\sqrt[3]{a b}},-\frac{1}{a} \cosh \frac{t}{\sqrt[3]{a b}}\right)$

assim a curvatura afim é,

$$
k_{a}(s)=\left|\gamma^{\prime \prime}(s), \gamma^{\prime \prime \prime}(s)\right|=-(a b)^{-\frac{2}{3}}
$$

Exemplo 3.38. A curvatura afim da parábola $y=x^{2}$ é zero.

De fato, consideremos a parametrização $\alpha(t)=\left(t, t^{2}\right)$, por diferenciação

$$
\dot{\alpha}(t)=(1,2 t) \text { e } \ddot{\gamma}(t)=(0,2)
$$

Logo $|\dot{\alpha}(t), \ddot{\alpha}(t)|=2$. Por outro lado considerando $t_{0}=0$, então o difeomorfismo $\sigma$ é dado pela relação $\sigma(t)=\sqrt{2} t$ onde sua inversa é $\sigma^{-1}(s)=2^{-\frac{1}{3}} s$. Assim

$$
\gamma(s)=\left(\alpha \circ \sigma^{-1}\right)(s)=\left(\frac{s}{\sqrt[3]{2}}, \frac{s^{2}}{\sqrt[3]{4}}\right)
$$

$\gamma^{\prime}(s)=\left(\frac{1}{\sqrt[3]{2}}, \frac{2 s}{\sqrt[3]{4}}\right), \gamma^{\prime \prime}(s)=\left(0, \frac{2}{\sqrt[3]{4}}\right)$ e $\gamma^{\prime \prime \prime}(s)=(0,0)$. Portanto

$$
k_{a}(s)=\left|\gamma^{\prime \prime}(s), \gamma^{\prime \prime \prime}(s)\right|=0
$$

Notemos que a curvatura afim da elipse, hipérbole e parábola são funções constante. 


\subsection{Tangentes afins e normais afins}

Definição 3.39. Seja $\gamma: I \rightarrow \mathbb{R}^{2}$ uma curva plana não degenerada parametrizada por comprimento de arco afim. O vetor $\gamma^{\prime}\left(s_{0}\right)$ é chamado vetor tangente afim a $\gamma$ em $\gamma\left(s_{0}\right)$. O vetor $\gamma^{\prime \prime}\left(s_{0}\right)$ é chamado vetor normal afim a $\gamma$ em $\gamma\left(s_{0}\right)$.

Definição 3.40. Seja $\gamma: I \rightarrow \mathbb{R}^{2}$ uma curva não degenerada parametrizada por comprimento de arco afim.

- Suponha $k_{a}\left(s_{0}\right) \neq 0$. Então o ponto $\gamma\left(s_{0}\right)+\frac{1}{k_{a}\left(s_{0}\right)} \gamma^{\prime \prime}\left(s_{0}\right)$ é chamado centro de curvatura afim de $\gamma$ em $\gamma\left(s_{0}\right)$.

- A evoluta afim é definida como o lugar geométrico de centros de curvatura afim.

- A curva $\gamma^{\prime \prime}: I \rightarrow \mathbb{R}^{2}$ é chamada de curva normal afim.

Definição 3.41. Seja $\gamma: I \rightarrow \mathbb{R}^{2}$ uma curva plana não degenerada parametrizada por comprimento de arco afim. A curva $\gamma$ tem uma inflexão afim em $\gamma\left(s_{0}\right)$ se $k_{a}\left(s_{0}\right)=0$. Uma inflexão afim é chamada ordinária se $k_{a}^{\prime}\left(s_{0}\right) \neq 0$. Uma inflexão afim é chamada degenerada se $k_{a}^{\prime}\left(s_{0}\right)=0$.

Definição 3.42. Seja $\gamma: I \rightarrow \mathbb{R}^{2}$ uma curva plana não degenerada parametrizada por comprimento de arco afim. A curva $\gamma$ tem um vértice afim em $\gamma\left(s_{0}\right)$ se $k_{a}\left(s_{0}\right) \neq 0$ e $k_{a}^{\prime}\left(s_{0}\right)=0$. Um vértice afim é chamado ordinário se $k_{a}^{\prime \prime}\left(s_{0}\right) \neq 0$. Um vértice afim é chamada degenerado se, $k_{a}^{\prime \prime}\left(s_{0}\right)=0$.

No que segue apresentamos resultados que exibem a relação de conceitos da geometria afim com os da geometria Euclidiana, tais como comprimento de arco afim e normal afim.

Lema 3.43. Seja $\gamma: I \rightarrow \mathbb{R}^{2}$ uma curva parametrizada por comprimento de arco e seja $\sigma$ uma função comprimento de arco afim, escrevendo $\xi=\sigma^{-1}: J \rightarrow I$ e pondo $t=\xi(s)$, então a curva $\alpha=\gamma \circ \xi$ está parametrizada por comprimento de arco afim e satisfaz as seguintes relações, onde $k$ é a curvatura de $\gamma$.

1. $\frac{d \xi}{d s}(s)=\frac{d t}{d s}(s)=k(t)^{-\frac{1}{3}}$.

2. $\frac{d^{2} \xi}{d s^{2}}(s)=\frac{d^{2} t}{d s^{2}}(s)=-\frac{1}{3} k(t)^{-\frac{5}{3}} \dot{k}(t)$.

3. $\frac{d^{3} \xi}{d s^{3}}(s)=\frac{d^{3} t}{d s^{3}}(s)=\frac{5}{9} k(t)^{-3} \dot{k}(t)^{2}-\frac{1}{3} k(t)^{-2} \ddot{k}(t)$. 
Demonstração: Por hipótese temos $\alpha(s)=\gamma(\xi(s))$. Logo por diferenciação tem-se $\alpha^{\prime}(s)=\dot{\gamma}(\xi(s))\left(\xi^{\prime}(s)\right)$. Por outro lado, $\dot{\gamma}(\xi(s))$ é linear e, identificando da forma usual $\dot{\gamma}(\xi(s))(1)=\dot{\gamma}(\xi(s))$, obtemos $\alpha^{\prime}(s)=\xi^{\prime}(s) \dot{\gamma}(\xi(s))$ daí,

$$
\alpha^{\prime}(s)=\xi^{\prime}(s) \dot{\gamma}(t)
$$

Por diferenciação $\quad \alpha^{\prime \prime}(s)=\xi^{\prime \prime}(s) \dot{\gamma}(\xi(s))+\xi^{\prime}(s) \ddot{\gamma}(\xi(s))\left(\xi^{\prime}(s)\right)$ e, com os argumentos recem mencionados (linearidade da derivada e identificando a derivada com um vetor), tem-se

$$
\alpha^{\prime \prime}(s)=\xi^{\prime \prime}(s) \dot{\gamma}(t)+\xi^{\prime}(s)^{2} \ddot{\gamma}(t)
$$

1. Por hipótese $\left|\alpha^{\prime}(s), \alpha^{\prime \prime}(s)\right|=1$, substituindo as relações acima tem-se

$$
\left|\xi^{\prime}(s) \dot{\gamma}(\xi(s)), \xi^{\prime \prime}(s) \dot{\gamma}(\xi(s))+\xi^{\prime}(s)^{2} \ddot{\gamma}(\xi(s))\right|=1 .
$$

Pela linearidade do determinante,

$$
1=\xi^{\prime}(s) \xi^{\prime \prime}(s)|\dot{\gamma}(\xi(s)), \dot{\gamma}(\xi(s))|+\xi^{\prime}(s) \xi^{\prime}(s)^{2}|\dot{\gamma}(\xi(s)), \ddot{\gamma}(\xi(s))|,
$$

logo, como $|\dot{\gamma}(\xi(s)), \dot{\gamma}(\xi(s))|=|\dot{\gamma}(t), \dot{\gamma}(t)|=0$, então

$$
1=\xi^{\prime}(s)^{3}|\dot{\gamma}(t), \ddot{\gamma}(t)|=\xi^{\prime}(s)^{3} k(t) \text { portanto } \xi^{\prime}(s)=k(t)^{-\frac{1}{3}} .
$$

2. $\frac{d^{2} \xi}{d s^{2}}(s)=\frac{d}{d s}\left(\frac{d \xi}{d s}(s)\right)=\frac{d}{d s}\left(k(\xi(s))^{-\frac{1}{3}}\right)=-\frac{1}{3} k(t)^{-\frac{4}{3}} \dot{k}(t) \dot{\xi}(s)$, substituindo $\dot{\xi}(s)=k(t)^{-\frac{1}{3}}$ na relação acima obtemos $\frac{d^{2} \xi}{d s^{2}}(s)=-\frac{1}{3} k(t)^{-\frac{5}{3}} \dot{k}(t)$.

3. $\frac{d^{3} \xi}{d s^{3}}(s)=\frac{d}{d s}\left(\frac{d^{2} \xi}{d s^{2}}(s)\right)=\frac{d}{d s}\left(-\frac{1}{3} k(\xi(s))^{-\frac{5}{3}} \dot{k}(\xi(s))\right)$,

$$
\begin{aligned}
& \frac{d^{3} \xi}{d s^{3}}(s)=-\frac{1}{3}\left(-\frac{5}{3} k(t)^{-\frac{8}{3}} \dot{k}(t)^{2} \dot{\xi}(s)+k(t)^{-\frac{5}{3}} \ddot{k}(t) \dot{\xi}(s)\right), \\
& \frac{d^{3} \xi}{d s^{3}}(s)=\frac{5}{9} k(t)^{-\frac{8}{3}} \dot{k}(t)^{2} k(t)^{-\frac{1}{3}}-\frac{1}{3} k(t)^{-\frac{5}{3}} \ddot{k}(t) k(t)^{-\frac{1}{3}},
\end{aligned}
$$

portanto,

$$
\frac{d^{3} \xi}{d s^{3}}(s)=\frac{5}{9} k(t)^{-3} \dot{k}(t)^{2}-\frac{1}{3} k(t)^{-2} \ddot{k}(t)
$$


Observação 3.44. Seja $\gamma: I \rightarrow \mathbb{R}^{2}$ uma curva plana parametrizada por comprimento de arco. Então tem-se as seguintes relações:

1. $|\ddot{\gamma}(t), \dddot{\gamma}(t)|=k(t)^{3}, \forall t \in I$

2. $|\dot{\gamma}(t), \dddot{\gamma}(t)|=\dot{k}(t)$, se $k(t) \neq 0$.

Com efeito, como $\gamma$ está parametrizada por comprimento de arco, $T(t)=\dot{\gamma}(t),(\mathrm{T}(\mathrm{t})$ vetor tangente e N(t) vetor normal). Dadas as equações de Frenet-Serret

$$
\dot{T}(t)=k(t) N(t) \text { e } \dot{N}(t)=-k(t) T(t)
$$

temos $\ddot{\gamma}(t)=\dot{T}(t)=k(t) N(t)$. Por diferenciação $\dddot{\gamma}(t)=\dot{k}(t) N(t)+k(t) \dot{N}(t)$. Substituindo as equações Serret-Frenet obtemos $\dddot{\gamma}(t)=\dot{k}(t) N(t)+k(t)(-k(t) \dot{\gamma}(t))$ e, portanto,

$$
\dddot{\gamma}(t)=\dot{k}(t) N(t)-k(t)^{2} \dot{\gamma}(t)
$$

Para demonstrar o item 1, usamos as relações acima

$$
|\ddot{\gamma}(t), \dddot{\gamma}(t)|=\left|k(t) N(t), \dot{k}(t) N(t)-k(t)^{2} \dot{\gamma}(t)\right|
$$

usando linearidade do determinante

$$
|\ddot{\gamma}(t), \dddot{\gamma}(t)|=-k(t)^{2}|k(t) N(t), \dot{\gamma}(t)|
$$

$\operatorname{assim}|\ddot{\gamma}(t), \dddot{\gamma}(t)|=-k(t)^{2}|\ddot{\gamma}(t), \dot{\gamma}(t)|$ e, portanto,

$$
|\ddot{\gamma}(t), \dddot{\gamma}(t)|=k(t)^{2}|\dot{\gamma}(t), \ddot{\gamma}(t)|=k(t)^{3}
$$

Analogamente para o item 2, substituimos as relações acima

$$
|\dot{\gamma}(t), \dddot{\gamma}(t)|=\left|\dot{\gamma}(t), \dot{k}(t) N(t)-k(t)^{2} \dot{\gamma}(t)\right|
$$

logo, pela linearidade do determinante, temos $|\dot{\gamma}(t), \dddot{\gamma}(t)|=\dot{k}(t)|\dot{\gamma}(t), N(t)|$. Por outro lado, como $k(t) \neq 0, N(t)=\frac{1}{k(t)} \ddot{\gamma}(t)$ daí

$$
|\dot{\gamma}(t), \dddot{\gamma}(t)|=\dot{k}(t)\left|\dot{\gamma}(t), \frac{1}{k(t)} \ddot{\gamma}(t)\right|
$$

portanto $|\dot{\gamma}(t), \dddot{\gamma}(t)|=\frac{\dot{k}(t)}{k(t)}|\dot{\gamma}(t), \ddot{\gamma}(t)|=\dot{k}(t)$ 
O seguinte teorema fornece uma relação para a curvatura afim em função de curvatura Euclidiana.

Teorema 3.45. Seja $\gamma: I \rightarrow \mathbb{R}^{2}$ uma curva parametrizada por comprimento de arco. Então a curvatura afim $k_{a}(s)$ é dada pela seguinte relação

$$
k_{a}(s)=k(t)^{\frac{4}{3}}-\frac{5}{9} k(t)^{-\frac{8}{3}} \dot{k}(t)^{2}+\frac{1}{3} k(t)^{-\frac{5}{3}} \ddot{k}(t) .
$$

Demonstração: Pelo Teorema (3.29), existe $\xi: J \rightarrow I$ difeomorfismo tal que a curva $\alpha$ : $J \rightarrow \mathbb{R}^{2}, \alpha(s)=\gamma(\xi(s))$ está parametrizada pelo comprimento de arco afim. Escrevendo $\xi(s)=t$, pela regra da cadeia temos $\alpha^{\prime}(s)=\dot{\gamma}(\xi(s))\left(\xi^{\prime}(s)\right)$. Usando linearidade da derivada e identificando $\dot{\gamma}(\xi(s))$ com $\dot{\gamma}(\xi(s))(1)$ tem-se

$$
\begin{gathered}
\alpha^{\prime}(s)=\xi^{\prime}(s) \dot{\gamma}(t), \\
\alpha^{\prime \prime}(s)=\xi^{\prime \prime}(s) \dot{\gamma}(t)+\xi^{\prime}(s)^{2} \ddot{\gamma}(t), \\
\alpha^{\prime \prime \prime}(s)=\xi^{\prime \prime \prime}(s) \dot{\gamma}(t)+3 \xi^{\prime}(s) \xi^{\prime \prime}(s) \ddot{\gamma}(t)+\xi^{\prime}(s)^{3} \dddot{\gamma}(t) .
\end{gathered}
$$

Por outro lado $k_{a}(s)=\left|\alpha^{\prime \prime}(s), \alpha^{\prime \prime \prime}(s)\right|$, substituindo as relações acima,

$$
\begin{aligned}
& k_{a}(s)=\left|\xi^{\prime \prime}(s) \dot{\gamma}(t)+\xi^{\prime}(s)^{2} \ddot{\gamma}(t), \xi^{\prime \prime \prime}(s) \dot{\gamma}(t)+3 \xi^{\prime}(s) \xi^{\prime \prime}(s) \ddot{\gamma}(t)+\xi^{\prime}(s)^{3} \dddot{\gamma}(t)\right|, \\
& =3 \xi^{\prime}(s) \xi^{\prime \prime}(s)^{2}|\dot{\gamma}(t), \ddot{\gamma}(t)|+\xi^{\prime}(s)^{3} \xi^{\prime \prime}(s)|\dot{\gamma}(t), \dddot{\gamma}(t)|+\xi^{\prime}(s)^{2} \xi^{\prime \prime \prime}(s)|\ddot{\gamma}(t), \dot{\gamma}(t)|+\xi^{\prime}(s)^{5}|\ddot{\gamma}(t), \dddot{\gamma}(t)|, \\
& =3 \xi^{\prime}(s) \xi^{\prime \prime}(s)^{2}|\dot{\gamma}(t), \ddot{\gamma}(t)|+\xi^{\prime}(s)^{3} \xi^{\prime \prime}(s)|\dot{\gamma}(t), \dddot{\gamma}(t)|+\xi^{\prime}(s)^{2} \xi^{\prime \prime \prime}(s)|\ddot{\gamma}(t), \dot{\gamma}(t)|+\xi^{\prime}(s)^{5}|\ddot{\gamma}(t), \dddot{\gamma}(t)| .
\end{aligned}
$$

Logo, das relações da Observação (3.44) e Lema (3.43) tem-se,

$$
\begin{gathered}
k_{a}(s)=3 k(t)^{-\frac{1}{3}}\left\{-\frac{1}{3} k(t)^{-\frac{5}{3}} \dot{k}(t)\right\} k(t)+k(t)\left\{-\frac{1}{3} k(t)^{-\frac{5}{3}} \dot{k}(t)\right\} \dot{k}(t)-k(t) k(t)^{-\frac{2}{3}}\left\{\frac{5}{9} k(t)^{-3} \dot{k}(t)^{2}\right. \\
\left.-\frac{1}{3} k(t)^{-2} \ddot{k}(t)\right\}+k(t)^{-\frac{5}{3}} k(t)^{3} \\
k_{a}(s)=\frac{1}{3} k(t)^{-\frac{1}{3}} k(t)^{-\frac{10}{3}} \dot{k}(t)^{2} k(t)-\frac{1}{3} k(t)^{-1} k(t)^{-\frac{5}{3}} \dot{k}(t)^{2}-\frac{5}{9} k(t) k(t)^{-\frac{2}{3}} k(t)^{-3} \dot{k}(t)^{2}+ \\
+\frac{1}{3} k(t) k(t)^{-\frac{2}{3}} k(t)^{-2} \ddot{k}(t)+k(t)^{\frac{4}{3}} \\
k_{a}(s)=\frac{1}{3} k(t)^{-\frac{8}{3}} \dot{k}(t)^{2}-\frac{1}{3} k(t)^{-\frac{8}{3}} \dot{k}(t)^{2}-\frac{5}{9} k(t)^{-\frac{8}{3}} \dot{k}(t)^{2}+\frac{1}{3} k(t)^{-\frac{5}{3}} \ddot{k}(t)+k(t)^{\frac{4}{3}} \\
k_{a}(s)=k(t)^{\frac{4}{3}}-\frac{5}{9} k(t)^{-\frac{8}{3}} \dot{k}(t)^{2}+\frac{1}{3} k(t)^{-\frac{5}{3}} \ddot{k}(t) .
\end{gathered}
$$


Observemos que, a equação (3.5) e o Lema (3.43) fornecem uma expressão para o vetor tangente afim em termos de um parâmetro arbitrario $t, \gamma^{\prime}(s)=k(t)^{-\frac{1}{3}} \dot{\gamma}(t)$.

Definição 3.46. Seja $\gamma: I \rightarrow \mathbb{R}^{2}$ uma curva não degenerada (não necessariamente parametrizada pelo comprimento de arco afim), definimos a curva normal afim como a curva $\gamma^{\prime \prime}: I \rightarrow \mathbb{R}^{2}$, onde o simbolo' representa a derivação em relação ao parametro comprimento de arco afim.

Observemos que $\gamma^{\prime \prime}(s)=\alpha^{\prime \prime}(s)$, onde $\alpha=\gamma \circ \xi$.

Proposição 3.47. Seja $\gamma: I \rightarrow \mathbb{R}^{2}$ uma curva plana não degenerada parametrizada por comprimento de arco. Então a curva normal afim está dada pela relação seguinte:

$$
\gamma^{\prime \prime}(s)=-\frac{1}{3} k(t)^{-\frac{5}{3}} \dot{k}(t) T(t)+k(t)^{\frac{1}{3}} N(t)
$$

Demonstração: Pelo Corolário (3.31) existe $\xi: J \rightarrow I$ difeomorfismo tal que a curva $\alpha: J \rightarrow \mathbb{R}^{2}, \alpha(s)=\gamma(\xi(s))$ é parametrizada pelo comprimento de arco afim. Escrevemos $\xi(s)=t$, nestas condições $T(t)=\dot{\gamma}(t)$ e $\ddot{\gamma}(t)=\dot{T}(\xi(s)) \xi^{\prime}(s)$, isto é

$$
\dot{\gamma}(t)=T(t) \text { e } \ddot{\gamma}(t)=\dot{T}(t) k(t)^{-\frac{1}{3}}
$$

Por outro lado, da equação (3.6) $\alpha^{\prime \prime}(s)=\xi^{\prime \prime}(s) \dot{\gamma}(t)+\xi^{\prime}(s)^{2} \ddot{\gamma}(t)$ e pelas relações do Lema $(3.43)$

$$
\alpha^{\prime \prime}(s)=-\frac{1}{3} \dot{k}(t) k(t)^{-\frac{5}{3}} \dot{\gamma}(t)+k(t)^{-\frac{2}{3}} \ddot{\gamma}(t)
$$

Como $\dot{\gamma}(t)=T(t)$ e $\ddot{\gamma}(t)=k(t) N(t)$ então conclui-se,

$$
\alpha^{\prime \prime}(s)=-\frac{1}{3} \dot{k}(t) k(t)^{-\frac{5}{3}} T(t)+k(t)^{\frac{1}{3}} N(t) .
$$

Observação 3.48. Se $\gamma: I \rightarrow \mathbb{R}^{2}$ é uma curva plana não degenerada (não necessariamente parametrizada por comprimento de arco afim) então, da proposição acima, $-\frac{1}{3} \dot{k}(t) k(t)^{-\frac{5}{3}} \dot{\gamma}(t)+k(t)^{-\frac{2}{3}} \ddot{\gamma}(t)$ fornece uma expressão para o vetor normal afim em termos do parâmetro comprimento de arco de $\gamma$.

\subsection{Reta tangente afim e reta normal afim}

Sejam $\gamma: I \rightarrow \mathbb{R}^{2}$ uma curva não degenerada parametrizada por comprimento de arco e $\sigma: I \rightarrow J$ uma função comprimento de arco afim. Considerando a mudança de 
parâmetro $\xi=\sigma^{-1}: J \rightarrow I \operatorname{com} t=\xi(s)$ a curva $\alpha=\gamma \circ \xi$ está parametrizada por comprimento de arco afim.

Definição 3.49. A reta tangente afim a $\gamma$ em $\gamma\left(t_{0}\right)$ é a reta paralela ao vetor $\gamma^{\prime}\left(\xi\left(s_{0}\right)\right)$ que passa por $\gamma\left(s_{0}\right)$, onde $\xi\left(s_{0}\right)=t_{0}$.

Como $\gamma^{\prime}(s)=k(t)^{-\frac{1}{3}} \dot{\gamma}(t)$ (equação (3.5)) temos que o vetor tangente afim é paralelo ao vetor tangente usual, com isto para $\gamma: I \rightarrow \mathbb{R}^{2} \operatorname{com} \gamma(t)=\left(x_{1}(t), x_{2}(t)\right)$ a reta tangente afim é dada por

$$
\left(x_{1}-x_{1}\left(t_{0}\right)\right) \dot{x_{2}}\left(t_{0}\right)-\left(x_{2}-x_{2}\left(t_{0}\right)\right) \dot{x_{1}}\left(t_{0}\right)=0,
$$

a qual fornece a seguinte equação matricial.

$$
\left\{x \in \mathbb{R}^{2}:\left|x-\gamma\left(t_{0}\right), \dot{\gamma}\left(t_{0}\right)\right|=0\right\}
$$

Definição 3.50. A reta normal afim a $\gamma$ em $\gamma\left(t_{0}\right)$ é a reta paralela a $\gamma^{\prime \prime}\left(\xi\left(s_{0}\right)\right)$ que passa por $\gamma\left(t_{0}\right)$, onde $t_{0}=\xi\left(s_{0}\right)$.

Proposição 3.51. Seja $\gamma: I \rightarrow \mathbb{R}^{2}$ uma curva plana não degenerada com $\gamma(t)=$ $(X(t), Y(t)), \forall t \in I$. Então a equação para a reta normal afim para $\gamma$ é dada pela seguinte equação:

$$
(y-Y(t))(\dot{k}(t) \dot{X}(t)-3 k(t) \ddot{X}(t))+(x-X(t))(3 k(t) \ddot{Y}(t)-\dot{k}(t) \dot{Y}(t))=0
$$

Demonstração: Desde a observação (3.48)

$$
\gamma^{\prime \prime}(s)=-\frac{1}{3} \dot{k}(t) k(t)^{-\frac{5}{3}} \dot{\gamma}(t)+k(t)^{-\frac{2}{3}} \ddot{\gamma}(t)
$$

onde $t=\xi(s)$, agora escrevemos da forma vetorial

$$
\gamma^{\prime \prime}(s)=\frac{1}{3} k(t)^{-\frac{5}{3}}(3 k(t) \ddot{X}(t)-\dot{k}(t) \dot{X}(t), 3 k(t) \ddot{Y}(t)-\dot{k}(t) \dot{Y}(t)) .
$$

Portanto a reta tocando o ponto $\gamma(t)$ a qual é paralela para $\gamma^{\prime \prime}(s)$ é fornecida pela seguinte equação

$$
\frac{1}{3} k(t)^{-\frac{5}{3}} \operatorname{det}\left(\begin{array}{cc}
3 k(t) \ddot{X}(t)-\dot{k}(t) \dot{X}(t) & 3 k(t) \ddot{Y}(t)-\dot{k}(t) \dot{Y}(t) \\
x-X(t) & y-Y(t)
\end{array}\right)=0 .
$$




\subsection{Transformação equiafim}

A geometria equiafim estuda propriedades invariantes por tranformações equiafins. O plano afim $\mathbb{R}^{2}$ utiliza um sistema de coordenadas denotado por $\{x, y\}$.

Definição 3.52. Uma tranformação equiafimde $\mathbb{R}^{2}$ é uma função $f: \mathbb{R}^{2} \rightarrow \mathbb{R}^{2}$ definida como segue $(x, y) \mapsto(\bar{x}, \bar{y})$ onde:

$$
\begin{aligned}
& \bar{x}(x, y)=a x+b y+p \\
& \bar{y}(x, y)=c x+d y+q
\end{aligned}
$$

tal que $\operatorname{det}\left(\begin{array}{ll}a & b \\ c & d\end{array}\right)=1$.

Teorema 3.53. O parâmetro comprimento de arco afim e a curvatura afim são invariantes por transformações equiafins.

Demonstração: Seja $f: \mathbb{R}^{2} \rightarrow \mathbb{R}^{2}$ uma transformação equiafim, isto é $(x, y) \mapsto(\bar{x}, \bar{y})$, onde

$$
\bar{x}(x, y)=a x+b y+p, \quad \bar{y}(x, y)=c x+d y+q .
$$

Dada $\gamma: I \rightarrow \mathbb{R}^{2}, \gamma(s)=(x(s), y(s))$ uma curva parametrizada por comprimento de arco afim, seja $\bar{\gamma}(s)=f(\gamma(s))$.

- $\bar{\gamma}$ esta parametrizada por comprimento de arco afim.

De fato, $\bar{\gamma}(s)=(\bar{x}(s), \bar{y}(s))$,

$$
\begin{aligned}
\bar{x}(s)=a x(s)+b y(s)+p, \quad \bar{y}(s)=c x(s)+d y(s)+q, \\
\left|\bar{\gamma}^{\prime}(s), \bar{\gamma}^{\prime \prime}(s)\right|=\left|\begin{array}{ll}
\bar{x}^{\prime}(s) & \bar{x}^{\prime \prime}(s) \\
\bar{y}^{\prime}(s) & \bar{y}^{\prime \prime}(s)
\end{array}\right|=\left|\begin{array}{ll}
a x^{\prime}(s)+b y^{\prime}(s) & a x^{\prime \prime}(s)+b y^{\prime \prime}(s) \\
c x^{\prime}(s)+d y^{\prime}(s) & c x^{\prime \prime}(s)+d y^{\prime \prime}(s)
\end{array}\right| \\
\left|\bar{\gamma}^{\prime}(s), \bar{\gamma}^{\prime \prime}(s)\right|=\left|\left(\begin{array}{ll}
a & b \\
c & d
\end{array}\right)\left(\begin{array}{ll}
x^{\prime}(s) & x^{\prime \prime}(s) \\
y^{\prime}(s) & y^{\prime \prime}(s)
\end{array}\right)\right|=\left|\begin{array}{ll}
a & b \\
c & d
\end{array}\right|\left|\begin{array}{ll}
x^{\prime}(s) & x^{\prime \prime}(s) \\
y^{\prime}(s) & y^{\prime \prime}(s)
\end{array}\right|
\end{aligned}
$$

como $f$ é uma transformação equiafim $a d-b c=1$, daí

$$
\left|\bar{\gamma}^{\prime}(s), \bar{\gamma}^{\prime \prime}(s)\right|=\left|\gamma^{\prime}(s), \gamma^{\prime \prime}(s)\right|=1
$$

Portanto $\bar{\gamma}$ está parametrizada por comprimento de arco afim. 
- A curvatura afim da curva $\gamma$ em $s$ coincide com a curvatura de $\bar{\gamma}$ em $s$.

De fato,

$$
\begin{aligned}
\left|\bar{\gamma}^{\prime \prime}(s), \bar{\gamma}^{\prime \prime \prime}(s)\right| & =\left|\begin{array}{ll}
\bar{x}^{\prime \prime}(s) & \bar{x}^{\prime \prime \prime}(s) \\
\bar{y}^{\prime \prime}(s) & \bar{y}^{\prime \prime \prime}(s)
\end{array}\right|=\left|\begin{array}{cc}
a x^{\prime \prime}(s)+b y^{\prime \prime}(s) & a x^{\prime \prime \prime}(s)+b y^{\prime \prime \prime}(s) \\
c x^{\prime \prime}(s)+d y^{\prime \prime}(s) & c x^{\prime \prime \prime}(s)+d y^{\prime \prime \prime}(s)
\end{array}\right| \\
\left|\bar{\gamma}^{\prime \prime}(s), \bar{\gamma}^{\prime \prime \prime}(s)\right| & =\left|\left(\begin{array}{ll}
a & b \\
c & d
\end{array}\right)\left(\begin{array}{ll}
x^{\prime \prime}(s) & x^{\prime \prime \prime}(s) \\
y^{\prime \prime}(s) & y^{\prime \prime \prime}(s)
\end{array}\right)\right|=\left|\begin{array}{ll}
a & b \\
c & d
\end{array}\right|\left|\begin{array}{cc}
x^{\prime \prime}(s) & x^{\prime \prime \prime}(s) \\
y^{\prime \prime}(s) & y^{\prime \prime \prime}(s)
\end{array}\right| .
\end{aligned}
$$

Como $f$ é uma transformação equiafim segue

$$
\left|\bar{\gamma}^{\prime \prime}(s), \bar{\gamma}^{\prime \prime \prime}(s)\right|=\left|\gamma^{\prime \prime}(s), \gamma^{\prime \prime \prime}(s)\right|=k_{a}(s)
$$

$\operatorname{assim} k_{a}(\bar{\gamma})(s)=k_{a}(s)$

Observação 3.54. Se $\gamma: I \rightarrow \mathbb{R}^{2}$ é uma curva não degenerada, então a seguinte relação é satisfeita:

$$
\frac{d A}{d s}(s)=A(s)\left(\begin{array}{cc}
0 & -k_{a}(s) \\
1 & 0
\end{array}\right)
$$

onde $A(s)=\left[\alpha^{\prime}(s) \alpha^{\prime \prime}(s)\right] \in S L(2, \mathbb{R})$, com $\alpha=\gamma \circ \xi$ e $\gamma^{\prime \prime}(s) \operatorname{denota} \alpha^{\prime \prime}(s)$.

De fato, sejam $\gamma: I \rightarrow \mathbb{R}^{2}$ uma curva não degenerada e $\sigma: I \rightarrow J$ uma função comprimento de arco afim com $A(s)=\left[\gamma^{\prime}(s) \gamma^{\prime \prime}(s)\right] \in S L(2, \mathbb{R}), \forall s=\sigma(t)$.

Consideremos a matriz

$$
C(s)=A(s)^{-1} \frac{d A}{d s}(s) .
$$

Escrevemos $\gamma(s)=(x(s), y(s))$, daí $A(s)=\left(\begin{array}{ll}x^{\prime}(s) & x^{\prime \prime}(s) \\ y^{\prime}(s) & y^{\prime \prime}(s)\end{array}\right)$.

Por outro lado como $A(s) \in S L(2, \mathbb{R})$ então,

$$
\begin{aligned}
A(s)^{-1} & =\left(\begin{array}{cc}
y^{\prime \prime}(s) & -x^{\prime \prime}(s) \\
-y^{\prime}(s) & x^{\prime}(s)
\end{array}\right) \text { e } \frac{d A}{d s}(s)=\left(\begin{array}{cc}
x^{\prime \prime}(s) & x^{\prime \prime \prime}(s) \\
y^{\prime \prime}(s) & y^{\prime \prime \prime}(s)
\end{array}\right) \text { portanto, } \\
C(s) & =\left(\begin{array}{cc}
y^{\prime \prime}(s) x^{\prime \prime}(s)-x^{\prime \prime}(s) y^{\prime \prime}(s) & y^{\prime \prime}(s) x^{\prime \prime \prime}(s)-x^{\prime \prime}(s) y^{\prime \prime \prime}(s) \\
-y^{\prime}(s) x^{\prime \prime}(s)+x^{\prime}(s) y^{\prime \prime}(s) & -y^{\prime}(s) x^{\prime \prime \prime}(s)+x^{\prime}(s) y^{\prime \prime \prime}(s)
\end{array}\right)=\left(\begin{array}{cc}
0 & -k_{a}(s) \\
1 & 0
\end{array}\right) .
\end{aligned}
$$

O seguinte teorema é o análogo afim do teorema fundamental no plano Euclidiano, onde a unicidade é a menos de isometrias.

Teorema 3.55. Dada uma função suave $k: J \rightarrow \mathbb{R}$, então existe uma curva plana com 
$\sigma$ como parâmetro comprimento de arco afim e $k$ como curvatura afim. Uma tal curva é única salvo transformações equiafins do plano.

Demonstração: Ver ([14], p. 3).

Já tínhamos visto que as elipses, hipérboles e parábolas tem curvatura afim constante, o seguinte teorema diz que estas são as únicas com curvatura afim constante.

Teorema 3.56. Uma curva $\gamma: I \rightarrow \mathbb{R}^{2}$ não degenerada com curvatura afim $k_{a}$ constante é uma curva quadrática.

Demonstração: Ver ([14], p. 4).

\subsection{Funções suporte afim}

Nesta seção estudamos duas importantes famílias de funções sobre uma curva plana não degenerada as quais são importantes para o estudo das propriedades geométricas de uma curva.

\subsubsection{Funções distância ao cubo afim}

Definição 3.57. Seja $\gamma: I \rightarrow \mathbb{R}^{2}$ uma curva plana não degenerada parametrizada por comprimento de arco afim. A família de funções $F: I \times \mathbb{R}^{2} \rightarrow \mathbb{R}$ definida por

$$
F(s, x)=\left|\gamma^{\prime}(s), \gamma(s)-x\right|
$$

é chamada de função distância ao cubo afim sobre $\gamma$. A função $F$ fornece uma família de funções a dois-parâmetros definida sobre a curva $\gamma$.

Para $x_{0} \in \mathbb{R}^{2}$ e $s_{0} \in I$, a distância afim de $x_{0}$ a $\gamma\left(s_{0}\right)$ é $F\left(s_{0}, x_{0}\right)$.

Em [6] e [10] a função distância ao cubo afim $F$, também é chamada de família de funções distância afim.

Para cada $x \in \mathbb{R}^{2}$, definimos $F_{x}: I \rightarrow \mathbb{R} \operatorname{com} F_{x}(s)=F(s, x)$. Observemos que $F_{x}$ é uma função real suave.

Lema 3.58. Sejam $x \in \mathbb{R}^{2}$ e $F: I \times \mathbb{R}^{2} \rightarrow \mathbb{R}$ uma função distância ao cubo afim sobre $\gamma$, então satisfaz as seguintes relações:

(a) $F_{x}^{\prime}(s)=\left|\gamma^{\prime \prime}(s), \gamma(s)-x\right|$,

(b) $F_{x}^{\prime \prime}(s)=\left|\gamma^{\prime \prime \prime}(s), \gamma(s)-x\right|-1$, 
(c) $F_{x}^{\prime \prime \prime}(s)=\left|\gamma^{(i v)}(s), \gamma(s)-x\right|$,

(d) $F_{x}^{(i v)}(s)=\left|\gamma^{(v)}(s), \gamma(s)-x\right|+\left|\gamma^{(i v)}(s), \gamma^{\prime}(s)\right|$.

Demonstração: Para a prova de cada um dos itens usamos a equação (3.2).

(a) Por diferenciação de $F_{x}$ tem-se $F_{x}^{\prime}(s)=\left|\gamma^{\prime \prime}(s), \gamma(s)-x\right|+\left|\gamma^{\prime}(s), \gamma\left(s^{\prime}\right)\right|$. Como $\left|\gamma^{\prime}(s), \gamma\left(s^{\prime}\right)\right|=0$ segue que $F_{x}^{\prime}(s)=\left|\gamma^{\prime \prime}(s), \gamma(s)-x\right|$.

(b) Por diferenciação de $F_{x}^{\prime}$, temos $F_{x}^{\prime \prime}(s)=\left|\gamma^{\prime \prime \prime}(s), \gamma(s)-x\right|+\left|\gamma^{\prime \prime}(s), \gamma^{\prime}(s)\right|$. Por outro lado como $\gamma$ está parametrizada pelo comprimento de arco afim, então $\left|\gamma^{\prime}(s), \gamma^{\prime \prime}(s)\right|=1$ daí, $F_{x}^{\prime \prime}(s)=\left|\gamma^{\prime \prime \prime}(s), \gamma(s)-x\right|-1$.

(c) Por diferenciação de $\left|\gamma^{\prime}(s), \gamma^{\prime \prime}(s)\right|=1$ segue que $\left|\gamma^{\prime}(s), \gamma^{\prime \prime \prime}(s)\right|=0$, agora diferenciando $F_{x}^{\prime \prime}$ tem-se $F_{x}^{\prime \prime \prime}(s)=\left|\gamma^{(i v)}(s), \gamma(s)-x\right|+\left|\gamma^{\prime \prime \prime}(s), \gamma^{\prime}(s)\right|$, portanto $F_{x}^{\prime \prime \prime}(s)=\left|\gamma^{(i v)}(s), \gamma(s)-x\right|$.

(d) Por diferenciação de $F_{x}^{\prime \prime \prime}$ obtemos $F_{x}^{(i v)}(s)=\left|\gamma^{(v)}(s), \gamma(s)-x\right|+\left|\gamma^{(i v)}(s), \gamma^{\prime}(s)\right|$.

A seguinte proposição caracteriza os pontos da evoluta e normal afim.

Proposição 3.59. Seja $\gamma: I \rightarrow \mathbb{R}^{2}$ uma curva plana não degenerada parametrizada por comprimento de arco afim. Então,

(a) $F_{x}^{\prime}\left(s_{0}\right)=0$ se, e somente se, existe $\lambda\left(s_{0}\right) \in \mathbb{R}$ tal que $\gamma\left(s_{0}\right)-x=\lambda\left(s_{0}\right) \gamma^{\prime \prime}\left(s_{0}\right)$.

(b) $F_{x}^{\prime}\left(s_{0}\right)=F_{x}^{\prime \prime}\left(s_{0}\right)=0$ se, e somente se, $k_{a}\left(s_{0}\right) \neq 0$ e $x=\gamma\left(s_{0}\right)+\frac{1}{k_{a}\left(s_{0}\right)} \gamma^{\prime \prime}\left(s_{0}\right)$.

(c) $F_{x}^{\prime}\left(s_{0}\right)=F_{x}^{\prime \prime}\left(s_{0}\right)=F_{x}^{\prime \prime \prime}\left(s_{0}\right)=0$ se, e somente se, $k_{a}\left(s_{0}\right) \neq 0, x=\gamma\left(s_{0}\right)+\frac{1}{k_{a}\left(s_{0}\right)} \gamma^{\prime \prime}\left(s_{0}\right)$ e $k_{a}^{\prime}\left(s_{0}\right)=0$.

(d) $F_{x}^{\prime}\left(s_{0}\right)=F_{x}^{\prime \prime}\left(s_{0}\right)=F_{x}^{\prime \prime \prime}\left(s_{0}\right)$ e $F_{x}^{(i v)}\left(s_{0}\right) \neq 0$ se, e somente se, $k_{a}\left(s_{0}\right) \neq 0, k_{a}^{\prime}\left(s_{0}\right)=0$, $x=\gamma\left(s_{0}\right)+\frac{1}{k_{a}\left(s_{0}\right)} \gamma^{\prime \prime}\left(s_{0}\right)$ e $k_{a}^{\prime \prime}\left(s_{0}\right) \neq 0$.

Demonstração: Observemos que $\gamma^{\prime \prime}\left(s_{0}\right) \neq 0$, pois $\gamma$ está parametrizada pelo comprimento de arco afim.

(a) Do Lema (3.58), $F_{x}^{\prime}\left(s_{0}\right)=0$ se, e somente se, $\left|\gamma^{\prime \prime}\left(s_{0}\right), \gamma\left(s_{0}\right)-x\right|=0$. Por outro lado, como $\gamma^{\prime \prime}\left(s_{0}\right) \neq 0$ temos $\left|\gamma^{\prime \prime}\left(s_{0}\right), \gamma\left(s_{0}\right)-x\right|=0$ se, e somente se, $\exists \lambda\left(s_{0}\right) \in \mathbb{R}$ tal que $\gamma\left(s_{0}\right)-x=\lambda\left(s_{0}\right) \gamma^{\prime \prime}\left(s_{0}\right)$.

(b) Do Lema (3.58) $F_{x}^{\prime \prime}\left(s_{0}\right)=0 \Leftrightarrow\left|\gamma^{\prime \prime \prime}\left(s_{0}\right), \gamma\left(s_{0}\right)-x\right|=1$. Pelo item (a) $F_{x}^{\prime}\left(s_{0}\right)=0 \Leftrightarrow$ existe $\lambda\left(s_{0}\right) \in \mathbb{R}$ tal que $\gamma\left(s_{0}\right)-x=\lambda\left(s_{0}\right) \gamma^{\prime \prime}\left(s_{0}\right)$. Assim, $F_{x}^{\prime}\left(s_{0}\right)=F_{x}^{\prime \prime}\left(s_{0}\right)=0 \Leftrightarrow\left|\gamma^{\prime \prime \prime}\left(s_{0}\right), \gamma\left(s_{0}\right)-x\right|=1$ e $\gamma\left(s_{0}\right)-x=\lambda\left(s_{0}\right) \gamma^{\prime \prime}\left(s_{0}\right)$ 


$$
\begin{aligned}
& \Leftrightarrow\left|\gamma^{\prime \prime \prime}\left(s_{0}\right), \lambda\left(s_{0}\right) \gamma^{\prime \prime}\left(s_{0}\right)\right|=1 \text { e } x=\gamma\left(s_{0}\right)-\lambda\left(s_{0}\right) \gamma^{\prime \prime}\left(s_{0}\right) \\
& \Leftrightarrow-\lambda\left(s_{0}\right) k_{a}\left(s_{0}\right)=1 \text { e } x=\gamma\left(s_{0}\right)-\lambda\left(s_{0}\right) \gamma^{\prime \prime}\left(s_{0}\right) \\
& \Leftrightarrow k_{a}\left(s_{0}\right) \neq 0, \lambda\left(s_{0}\right)=-\frac{1}{k_{a}\left(s_{0}\right)} \text { e } x=\gamma\left(s_{0}\right)-\lambda\left(s_{0}\right) \gamma^{\prime \prime}\left(s_{0}\right) \\
& \Leftrightarrow k_{a}\left(s_{0}\right) \neq 0 \text { e } x=\gamma\left(s_{0}\right)+\frac{1}{k_{a}\left(s_{0}\right)} \gamma^{\prime \prime}\left(s_{0}\right)
\end{aligned}
$$

Lembremos que o ponto definido pela equação abaixo é o centro de curvatura afim de $\gamma$ em $\gamma\left(s_{0}\right)$.

$$
x=\gamma\left(s_{0}\right)+\frac{1}{k_{a}\left(s_{0}\right)} \gamma^{\prime \prime}\left(s_{0}\right) .
$$

(c) Primeiro observemos que a derivada de $k_{a}(s)=\left|\gamma^{\prime \prime}(s), \gamma^{\prime \prime \prime}(s)\right|$ é fornecida pela equação

$$
k_{a}^{\prime}(s)=\left|\gamma^{\prime \prime}(s), \gamma^{(i v)}(s)\right|
$$

Agora do Lema (3.58), $F_{x}^{\prime \prime \prime}\left(s_{0}\right)=0$ se, e somente se, $\left|\gamma^{(i v)}\left(s_{0}\right), \gamma\left(s_{0}\right)-x\right|=0$. Usando o item(b), tem-se, $F_{x}^{\prime}\left(s_{0}\right)=F_{x}^{\prime \prime}\left(s_{0}\right)=F_{x}^{\prime \prime \prime}\left(s_{0}\right)=0 \Leftrightarrow k_{a}\left(s_{0}\right) \neq 0, x=\gamma\left(s_{0}\right)+\frac{1}{k_{a}\left(s_{0}\right)} \gamma^{\prime \prime}\left(s_{0}\right)$ $\mathrm{e}\left|\gamma^{(i v)}\left(s_{0}\right), \gamma\left(s_{0}\right)-x\right|=0$

$$
\begin{aligned}
& \Leftrightarrow k_{a}\left(s_{0}\right) \neq 0, x=\gamma\left(s_{0}\right)+\frac{1}{k_{a}\left(s_{0}\right)} \gamma^{\prime \prime}\left(s_{0}\right) \text { e }\left|\gamma^{(i v)}\left(s_{0}\right),-\frac{1}{k_{a}\left(s_{0}\right)} \gamma^{\prime \prime}\left(s_{0}\right)\right|=0, \\
& \Leftrightarrow k_{a}\left(s_{0}\right) \neq 0, x=\gamma\left(s_{0}\right)+\frac{1}{k_{a}\left(s_{0}\right)} \gamma^{\prime \prime}\left(s_{0}\right) \text { e } \quad-\frac{1}{k_{a}\left(s_{0}\right)}\left|\gamma^{(i v)}\left(s_{0}\right), \gamma^{\prime \prime}\left(s_{0}\right)\right|=0, \\
& \Leftrightarrow k_{a}\left(s_{0}\right) \neq 0, x=\gamma\left(s_{0}\right)+\frac{1}{k_{a}\left(s_{0}\right)} \gamma^{\prime \prime}\left(s_{0}\right) \text { e } \frac{1}{k_{a}\left(s_{0}\right)} k_{a}^{\prime}\left(s_{0}\right)=0, \\
& \Leftrightarrow k_{a}\left(s_{0}\right) \neq 0, x=\gamma\left(s_{0}\right)+\frac{1}{k_{a}\left(s_{0}\right)} \gamma^{\prime \prime}\left(s_{0}\right) \text { e } \quad k_{a}^{\prime}\left(s_{0}\right)=0 .
\end{aligned}
$$

(d) Por diferenciação de $k_{a}^{\prime}$ na equação (3.11) tem-se,

$$
k_{a}^{\prime \prime}(s)=\left|\gamma^{\prime \prime \prime}(s), \gamma^{(i v)}(s)\right|+\left|\gamma^{\prime \prime}(s), \gamma^{(v)}(s)\right|
$$

Por outro lado $\left|\gamma^{\prime}(s), \gamma^{\prime \prime}(s)\right|=1$ ( $\gamma$ está parametrizada por comprimento de arco afim), logo por diferenciação tem-se

$$
\left|\gamma^{\prime}(s), \gamma^{\prime \prime \prime}(s)\right|=0 \quad \text { e }\left|\gamma^{\prime \prime}(s), \gamma^{\prime \prime \prime}(s)\right|+\left|\gamma^{\prime}(s), \gamma^{(i v)}(s)\right|=0
$$

Portanto

$$
\left|\gamma^{\prime}(s), \gamma^{(i v)}(s)\right|=-k_{a}(s)
$$

Também $\left|\gamma^{\prime \prime \prime}(s), \gamma^{(i v)}(s)\right|=\left|-k_{a}(s) \gamma^{\prime}(s), \gamma^{(i v)}(s)\right|=-k_{a}(s)\left|\gamma^{\prime}(s), \gamma^{(i v)}(s)\right|$. Logo

$$
\left|\gamma^{\prime \prime \prime}(s), \gamma^{(i v)}(s)\right|=k_{a}(s)^{2}
$$


Substituindo (3.14) em (3.12) obtemos

$$
k_{a}^{\prime \prime}(s)=k_{a}(s)^{2}+\left|\gamma^{\prime \prime}(s), \gamma^{(v)}(s)\right|
$$

Do Lema $(3.58), F_{x}^{(i v)}\left(s_{0}\right)=0$ se, e somente se, $\left|\gamma^{(v)}\left(s_{0}\right), \gamma\left(s_{0}\right)-x\right|=-\left|\gamma^{(i v)}\left(s_{0}\right), \gamma^{\prime}\left(s_{0}\right)\right|$ agora pela equação (3.13),

$$
\left|\gamma^{(v)}\left(s_{0}\right), \gamma\left(s_{0}\right)-x\right|=-k_{a}\left(s_{0}\right)
$$

Agora $F_{x}^{\prime}\left(s_{0}\right)=F_{x}^{\prime \prime}\left(s_{0}\right)=F_{x}^{\prime \prime \prime}\left(s_{0}\right)=F_{x}^{(i v)}\left(s_{0}\right)=0 \Leftrightarrow k_{a}\left(s_{0}\right) \neq 0, x=\gamma\left(s_{0}\right)+\frac{1}{k_{a}\left(s_{0}\right)} \gamma^{\prime \prime}\left(s_{0}\right)$, $k_{a}^{\prime}\left(s_{0}\right)=0$ e $\left|\gamma^{(v)}\left(s_{0}\right), \gamma\left(s_{0}\right)-x\right|=-k_{a}\left(s_{0}\right)$,

$\Leftrightarrow x=\gamma\left(s_{0}\right)+\frac{1}{k_{a}\left(s_{0}\right)} \gamma^{\prime \prime}\left(s_{0}\right), k_{a}^{\prime}\left(s_{0}\right)=0$ e $\left|\gamma^{(v)}\left(s_{0}\right),-\frac{1}{k_{a}\left(s_{0}\right)} \gamma^{\prime \prime}\left(s_{0}\right)\right|=-k_{a}\left(s_{0}\right)$,

$\Leftrightarrow x=\gamma\left(s_{0}\right)+\frac{1}{k_{a}\left(s_{0}\right)} \gamma^{\prime \prime}\left(s_{0}\right), k_{a}^{\prime}\left(s_{0}\right)=0$ e $\left|\gamma^{(v)}\left(s_{0}\right), \gamma^{\prime \prime}\left(s_{0}\right)\right|=k_{a}\left(s_{0}\right)^{2}$,

$\Leftrightarrow x=\gamma\left(s_{0}\right)+\frac{1}{k_{a}\left(s_{0}\right)} \gamma^{\prime \prime}\left(s_{0}\right), k_{a}^{\prime}\left(s_{0}\right)=0$ e $\quad k_{a}\left(s_{0}\right)^{2}-k_{a}^{\prime \prime}\left(s_{0}\right)=k_{a}\left(s_{0}\right)^{2}$,

$\Leftrightarrow x=\gamma\left(s_{0}\right)+\frac{1}{k_{a}\left(s_{0}\right)} \gamma^{\prime \prime}\left(s_{0}\right), k_{a}^{\prime}\left(s_{0}\right)=0$ e $\quad k_{a}^{\prime \prime}\left(s_{0}\right)=0$.

Notemos que, da proposição acima tem-se as seguintes caracterizações:

- $F_{x_{0}}^{\prime}\left(s_{0}\right)=0$ se, e somente se, $x_{0}$ pertence à reta normal afim da curva $\gamma$ em $\gamma\left(s_{0}\right)$.

- $F_{x_{0}}^{\prime}\left(s_{0}\right)=F_{x_{0}}^{\prime \prime}\left(s_{0}\right)=0$ se, e somente se, $x_{0}$ pertence à evoluta afim.

Proposição 3.60. Seja $\gamma: I \rightarrow \mathbb{R}^{2}$ uma curva parametrizada por comprimento de arco afim. Então, $\forall x \in \mathbb{R}^{2}$, tem-se:

$$
x=\gamma(s)+F_{x}^{\prime}(s) \gamma^{\prime}(s)-F_{x}(s) \gamma^{\prime \prime}(s)
$$

Demonstração: Por hipótese $\gamma$ está parametrizada por comprimento de arco afim, $\operatorname{logo}\left|\gamma^{\prime}(s), \gamma^{\prime \prime}(s)\right|=1, \forall s \in I$, daí $\gamma^{\prime}(s)$ e $\gamma^{\prime \prime}(s)$ são linearmente independentes. Então existem famílias de funções $u, v: \mathbb{R}^{2} \times I \rightarrow \mathbb{R}$ tal que,

$$
x-\gamma(s)=u(x, s) \gamma^{\prime}(s)+v(x, s) \gamma^{\prime \prime}(s) .
$$

Por outro lado,

$$
\begin{aligned}
F_{x}(s) & =\left|x-\gamma(s), \gamma^{\prime}(s)\right|=\left|u(x, s) \gamma^{\prime}(s)+v(x, s) \gamma^{\prime \prime}(s), \gamma^{\prime}(s)\right| \\
& =u(x, s)\left|\gamma^{\prime}(s), \gamma^{\prime}(s)\right|+v(x, s)\left|\gamma^{\prime \prime}(s), \gamma^{\prime}(s)\right| \\
& =v(x, s)\left|\gamma^{\prime \prime}(s), \gamma^{\prime}(s)\right|
\end{aligned}
$$




$$
\begin{aligned}
& =-v(x, s) \\
F_{x}^{\prime}(s) & =\left|x-\gamma(s), \gamma^{\prime \prime}(s)\right|=\left|u(x, s) \gamma^{\prime}(s)+v(x, s) \gamma^{\prime \prime}(s), \gamma^{\prime \prime}(s)\right| \\
& =u(x, s)\left|\gamma^{\prime}(s), \gamma^{\prime \prime}(s)\right|+v(x, s)\left|\gamma^{\prime \prime}(s), \gamma^{\prime \prime}(s)\right| \\
& =u(x, s)\left|\gamma^{\prime}(s), \gamma^{\prime \prime}(s)\right| \\
& =u(x, s)
\end{aligned}
$$

Teorema 3.61. Sejam $\gamma: I \rightarrow \mathbb{R}^{2}$ uma curva não degenerada e $\sigma: I \rightarrow J$ uma função comprimento de arco afim. Se $\xi=\sigma^{-1}: J \rightarrow I$ e pondo $t=\xi(s)$, então a função distância ao cubo afim de $\alpha=\gamma \circ \xi$ é dada por:

$$
|x-\gamma(t), \dot{\gamma}(t)| k(t)^{-\frac{1}{3}}
$$

Demonstração: Pelo Lema (3.43) tem-se $\xi^{\prime}(s)=k(t)^{-\frac{1}{3}}$. Por diferenciação de $\alpha=\gamma \circ \xi$ temos $\alpha^{\prime}(s)=\dot{\gamma}(t) \xi^{\prime}(s)$. Daí $\alpha^{\prime}(s)=\dot{\gamma}(t) k(t)^{-\frac{1}{3}}$. Assim :

$$
\begin{aligned}
F(x, t) & =\left|x-\alpha(s), \alpha^{\prime}(s)\right| \\
& =\left|x-\gamma(t), \dot{\gamma}(t) \xi^{\prime}(s)\right| \\
& =|x-\gamma(t), \dot{\gamma}(t)| \xi^{\prime}(s) \\
& =|x-\gamma(t), \dot{\gamma}(t)| k(t)^{-\frac{1}{3}} .
\end{aligned}
$$

\subsubsection{Função altura afim}

Denotaremos como $\mathbb{S}^{1}$ o circulo unitário $\mathbb{S}^{1}=\left\{\left(x_{1}, x_{2}\right) \in \mathbb{R}^{2} / x_{1}^{2}+x_{2}^{2}=1\right\}$

Definição 3.62. Seja $\gamma: I \rightarrow \mathbb{R}^{2}$ uma curva plana não degenerada parametrizada por comprimento de arco afim, a família de funções suaves $H: I \times \mathbb{S}^{1} \rightarrow \mathbb{R}$, definida por

$$
H(s, u)=\left|\gamma^{\prime}(s), u\right|
$$

é chamada função altura afim sobre $\gamma$.

Para cada $u \in \mathbb{S}^{1}$ definimos $H_{u}: I \rightarrow \mathbb{R}$ como $H_{u}(s)=H(s, u)$. Observemos que, para cada $u$ fixo, $H_{u}$ é suave.

Lema 3.63. Seja $\gamma: I \rightarrow \mathbb{R}^{2}$ uma curva plana não degenerada parametrizada por comprimento de arco afim, então tem-se:

(a) $H_{u}^{\prime}(s)=\left|\gamma^{\prime \prime}(s), u\right|$,

(b) $H_{u}^{\prime \prime}(s)=\left|\gamma^{\prime \prime \prime}(s), u\right|$,

(c) $H_{u}^{\prime \prime \prime}(s)=\left|\gamma^{(i v)}(s), u\right|$. 
Demonstração: Segue por diferenciação.

Proposição 3.64. Seja $\gamma: I \rightarrow \mathbb{R}^{2}$ uma curva plana parametrizada pelo comprimento de arco afim então,

(a) $H_{u}^{\prime}\left(s_{0}\right)=0$ se, e somente se, existe $\lambda\left(s_{0}\right) \in \mathbb{R}$ tal que $u=\lambda\left(s_{0}\right) \gamma^{\prime \prime}\left(s_{0}\right)$,

(b) $H_{u}^{\prime}\left(s_{0}\right)=H_{u}^{\prime \prime}\left(s_{0}\right)=0$ se, e somente se, existe $\lambda\left(s_{0}\right) \in \mathbb{R}$ tal que $u=\lambda\left(s_{0}\right) \gamma^{\prime \prime}\left(s_{0}\right)$ e $k_{a}\left(s_{0}\right)=0, e$

(c) $H_{u}^{\prime}\left(s_{0}\right)=H_{u}^{\prime \prime}\left(s_{0}\right)=H_{u}^{\prime \prime \prime}\left(s_{0}\right)=0$ se, e somente se, existe $\lambda\left(s_{0}\right) \in \mathbb{R}$ tal que $u=$ $\lambda\left(s_{0}\right) \gamma^{\prime \prime}\left(s_{0}\right)$ e $k_{a}\left(s_{0}\right)=k_{a}^{\prime}\left(s_{0}\right)=0$.

Observemos que $\lambda(s)= \pm \frac{1}{\sqrt{x_{1}^{\prime \prime}(s)^{2}+x_{2}^{\prime \prime}(s)^{2}}}$ onde $\gamma(s)=\left(x_{1}(s), x_{2}(s)\right)$.

Demonstração: Por hipótese $\gamma$ está parametrizada pelo comprimento de arco afim. Então $\gamma^{\prime \prime}\left(s_{0}\right) \neq 0$.

(a) $H_{u}^{\prime}\left(s_{0}\right)=\left|\gamma^{\prime \prime}\left(s_{0}\right), u\right|$. Como $\gamma^{\prime \prime}\left(s_{0}\right) \neq 0$, então $\left|\gamma^{\prime \prime}\left(s_{0}\right), u\right|=0$ se, e somente se, existe $\lambda\left(s_{0}\right) \in \mathbb{R}$ tal que $u=\lambda\left(s_{0}\right) \gamma^{\prime \prime}\left(s_{0}\right)$. Além disso, se $\gamma(s)=\left(x_{1}(s), x_{2}(s)\right), \forall s \in I$ então, como $u \in \mathbb{S}^{1}$ tem-se, $\lambda\left(s_{0}\right)= \pm \frac{1}{\sqrt{x_{1}^{\prime \prime}\left(s_{0}\right)^{2}+x_{2}^{\prime \prime}\left(s_{0}\right)^{2}}}$.

(b) Pelo lema acima temos $H_{u}^{\prime \prime}\left(s_{0}\right)=0$ se, e somente se, $\left|\gamma^{\prime \prime \prime}\left(s_{0}\right), u\right|=0$. Por outro lado, usando o item (a), tem-se

$$
\begin{aligned}
H_{u}^{\prime}\left(s_{0}\right)=H_{u}^{\prime \prime}\left(s_{0}\right)=0 & \Leftrightarrow u=\lambda\left(s_{0}\right) \gamma^{\prime \prime}\left(s_{0}\right) \text { e }\left|\gamma^{\prime \prime \prime}\left(s_{0}\right), u\right|=0, \\
& \Leftrightarrow u=\lambda\left(s_{0}\right) \gamma^{\prime \prime}\left(s_{0}\right) \text { e }\left|\gamma^{\prime \prime \prime}\left(s_{0}\right), \lambda\left(s_{0}\right) \gamma^{\prime \prime}\left(s_{0}\right)\right|=0, \\
& \Leftrightarrow u=\lambda\left(s_{0}\right) \gamma^{\prime \prime}\left(s_{0}\right) \text { e } \lambda\left(s_{0}\right) k_{a}\left(s_{0}\right)=0, \\
& \Leftrightarrow u=\lambda\left(s_{0}\right) \gamma^{\prime \prime}\left(s_{0}\right) \text { e } k_{a}\left(s_{0}\right)=0 .
\end{aligned}
$$

(c) Pelo lema acima $H_{u}^{\prime \prime \prime}\left(s_{0}\right)=0$ se, e somente se, $\left|\gamma^{(i v)}\left(s_{0}\right), u\right|=0$. Por outro lado, usando o item (b) tem-se,

$$
\begin{aligned}
& H_{u}^{\prime}\left(s_{0}\right)=H_{u}^{\prime \prime}\left(s_{0}\right)=H_{u}^{\prime \prime \prime}\left(s_{0}\right)=H^{(i v)}\left(s_{0}\right)=0 \Leftrightarrow u=\lambda\left(s_{0}\right) \gamma^{\prime \prime}\left(s_{0}\right) \text { e } k_{a}\left(s_{0}\right)=0 \mathrm{e} \\
& \left|\gamma^{(i v)}\left(s_{0}\right), u\right|=0, \\
& \quad \Leftrightarrow u=\lambda\left(s_{0}\right) \gamma^{\prime \prime}\left(s_{0}\right) \text { e } k_{a}\left(s_{0}\right)=0 \text { e }\left|\gamma^{(i v)}\left(s_{0}\right), \lambda\left(s_{0}\right) \gamma^{\prime \prime}\left(s_{0}\right)\right|=0, \\
& \Leftrightarrow u=\lambda\left(s_{0}\right) \gamma^{\prime \prime}\left(s_{0}\right) \text { e } k_{a}\left(s_{0}\right)=0 \text { e } \lambda\left(s_{0}\right)\left|\gamma^{(i v)}\left(s_{0}\right), \gamma^{\prime \prime}\left(s_{0}\right)\right|=0, \\
& \left.\quad \Leftrightarrow u=\lambda\left(s_{0}\right) \gamma^{\prime \prime}\left(s_{0}\right) \text { e } k_{a}\left(s_{0}\right)=0 \text { e }-\lambda\left(s_{0}\right) k_{a}^{\prime}(s)=0 \text { (equação }(3.11)\right), \\
& \Leftrightarrow u=\lambda\left(s_{0}\right) \gamma^{\prime \prime}\left(s_{0}\right) \text { e } k_{a}\left(s_{0}\right)=0 \text { e } k_{a}^{\prime}(s)=0 .
\end{aligned}
$$




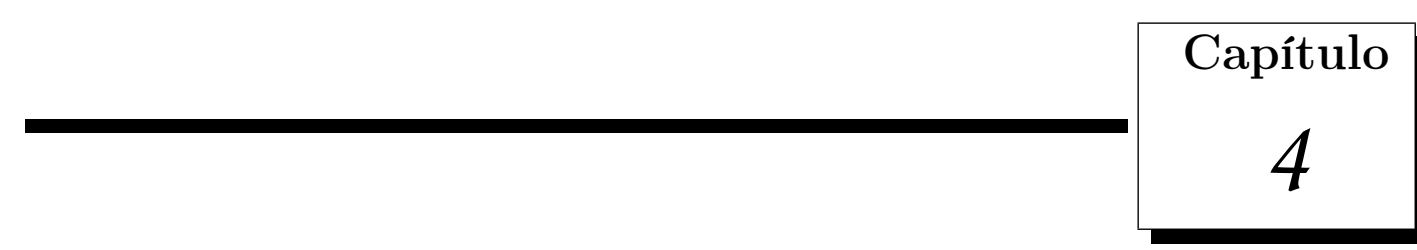

\section{Aberração}

Neste capítulo apresentamos uma interpretação geométrica para o contato entre duas curvas estudando a geometria das primeira, segunda e terceira derivada de uma função real de variável real. Tambem estudamos a ordem de contato de uma curva com uma cônica obtendo equações explícitas para a ordem de contato 4, 5 e 6 . Seguimos neste capítulo as notações e resultados desenvolvidos em Schot [15].

Para fixar a notação neste capítulo consideramos uma função suave $f: I \rightarrow \mathbb{R}$, onde $I$ é um intervalo aberto de $\mathbb{R}$. Falamos da curva $y=f(x)$ para representar a curva $\gamma: I \rightarrow \mathbb{R}^{2}$ definida por $\gamma(x)=(x, f(x)), \forall x \in I$.

\subsection{Interpretação geométrica da primeira derivada}

A intepretação da primeira derivada usa a inclinação da reta tangente à curva. Se $f: \mathbb{R} \rightarrow \mathbb{R}$ é uma função suave pondo $y=f(x)$, a primeira derivada satisfaz,

$$
y^{\prime}=\tan \psi=m
$$

onde: ' denota a diferenciação com respeito a $x$ e $\psi$ é o ângulo entre a reta tangente à curva e o eixo- $x$.

A reta tangente em $\mathrm{P}$ pode-se considerar como o limite de uma reta secante tocando $\mathrm{P}$ e um ponto vizinho $\mathrm{Q}$ na curva, quando $\mathrm{Q}$ tende para $\mathrm{P}$ (ver Figura (4.1)). Como a reta tangente é $y=g(x)=m\left(x-x_{0}\right)+f\left(x_{0}\right)$ então $g\left(x_{0}\right)=f\left(x_{0}\right), g^{\prime}\left(x_{0}\right)=m=f^{\prime}\left(x_{0}\right)$, assim a reta tangente tem ao menos 2-ponto de contato em $\mathrm{P}$ com a curva $y=f(x)$, (ver Definição (2.6)). 


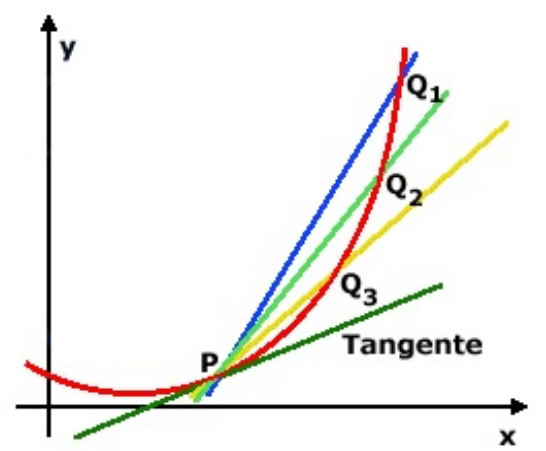

Figura 4.1: Reta tangente

\subsection{Interpretação geométrica da segunda derivada}

Seja $f: \mathbb{R} \rightarrow \mathbb{R}$ uma função suave, exprimindo $y=f(x)$. A segunda derivada $y^{\prime \prime}$ pode ser interpretada geometricamente em termos da curvatura. A curvatura de uma curva num ponto $\mathrm{P}, k(P)$ é dada pela razão de mudança da direção relativo ao parâmetro comprimento de arco no ponto $\mathrm{P}$ (ver equação (1.5), do Capítulo I).

$$
k=\frac{d \psi}{d s}=\frac{d \psi}{d x} \frac{d x}{d s}=\frac{y^{\prime \prime}}{1+\left(y^{\prime}\right)^{2}} \frac{1}{\sqrt{1+\left(y^{\prime}\right)^{2}}}=\frac{y^{\prime \prime}}{\left(1+\left(y^{\prime}\right)^{2}\right)^{\frac{3}{2}}}
$$

É conhecido que a curvatura $k$ é igual ao recíproco do raio de curvatura $\rho$, ou seja $k=\frac{1}{\rho}$, onde $\rho$ é o raio do círculo o qual tem a mesma curvatura no ponto que a curva.

O círculo limite de círculos tocando $\mathrm{P}$ e dois pontos vizinhos $P_{1}$ e $P_{2}$ na curva, quando $P_{1}$ e $P_{2}$ se aproximan a $P$ é chamado círculo osculador (ver Figura (4.2)).

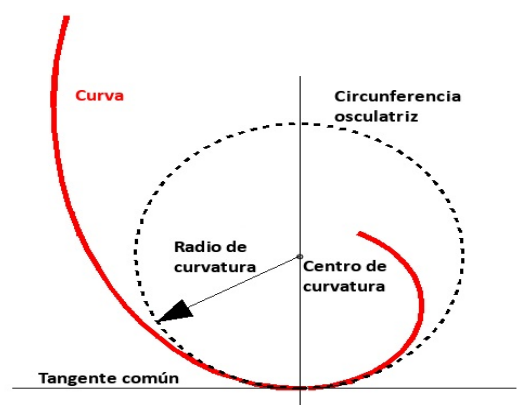

Figura 4.2: Círculo osculador

Alternativamente como as derivadas $y, y^{\prime}$ e $y^{\prime \prime}$ do círculo osculador são iguais às da 
curva em P. Logo segue da Definição (2.6) que o círculo osculador tem ao menos 3-ponto de contato em $\mathrm{P}$ com a curva $y=f(x)$.

Observação 4.1. Se $f: \mathbb{R} \rightarrow \mathbb{R}$ é uma função suave. O centro de curvatura 0 num ponto $P=\left(x_{0}, y_{0}\right)$, é definido como a posição limite do ponto de interseção de retas normais à curva $y=f(x)$ vizinhas a $P$. Se mostra que o raio de curvatura $\rho$ em $P$ é o comprimento do segmento de reta $P 0$.

\subsection{Interpretação geométrica da terceira derivada}

Para interpretar a primeira derivada usamos a inclinação da reta tangente à curva e também da reta tangente a curva no ponto $\mathrm{P}$, analogamente se fez para interpretar a segunda derivada usando o conceito de curvatura e do círculo osculador. Para apresentar interpretação geométrica da terceira derivada usamos o conceito de aberração e de parábola osculadora.

Definição 4.2. A aberração de uma curva num ponto $P$ é a tangente do ângulo $\delta$, onde $\delta$ é o ângulo formado entre a normal à curva em $P$ e a posição limite de uma reta que passa por $P$ e pelo ponto médio da corda paralela à reta tangente em $P$ com extremidades na curva, quando as cordas se aproximam a $P$.

Em [15] obtem-se a seguinte relação para a aberração,

$$
\tan \delta=\frac{\dot{\rho}}{3 \rho}
$$

onde $\rho$ é o raio de curvatura e o símbolo • indica a derivação em relação ao parâmetro $\psi, \operatorname{com} \psi$ o ângulo entre a reta tangente à curva e o eixo- $x$.

Definição 4.3. A parábola osculadora é a parábola por $P$ a qual é o limite das parábolas por $P$ e três pontos vizinhos $P_{1}, P_{2}$ e $P_{3}$ da curva, quando $P_{1}, P_{2}$ e $P_{3}$ se aproximan a $P$.

A aberração em $\mathrm{P}$ pode definir-se alternativamente como a tangente do ângulo entre o eixo da parábola osculadora e a normal para a curva em P.

Observemos que a aberração pode não existir, desde que a parábola osculadora não necessariamente existe.

Na Seção Cônica osculadora verificamos que a parábola osculadora tem ao menos 4-ponto de contato em $\mathrm{P}$ com a curva $y=f(x)$.

Para ter uma interpretação geométrica da aberração consideremos a figura (4.3). 


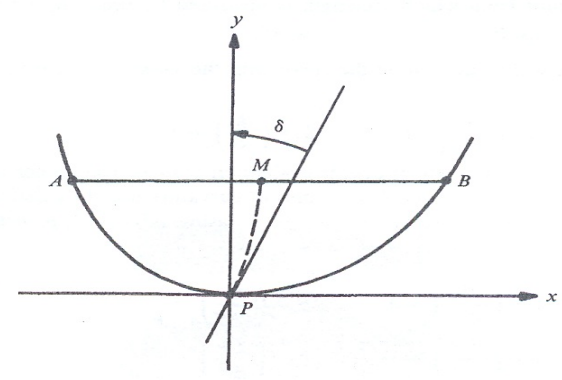

Figura 4.3: Interpretação da aberração

Isto é, suponhamos a curva seja tangente ao eixo- $x$ na origem $\mathrm{P}$, seja $A B$ a corda paralela para a tangente em $\mathrm{P}$ e $\mathrm{M}$ o ponto medio de $A B$. Como a corda paralela aproximase da tangente, o ângulo entre $P M$ e a normal aproxima um ângulo limite $\delta$. Este ângulo, se existe é chamado o ângulo de aberração e a posição limite de $P M$ é chamado o eixo de aberração.

Observação 4.4. Analogamente a definição de centro de curvatura, o centro de aberração $C$ de um ponto $P$, é definido como a posição limite do ponto de interseção de eixos de aberração vizinhas a $P$ e o comprimento da corda $P C$ é chamado raio de aberração e é denotado por $R$.

Em [15] usando relações trigonométricas elementares obtem-se uma relação para o raio de aberração .

$$
R=\frac{\rho \cos \delta}{1-\dot{\delta}}
$$

Considerando que a aberração existe, deduzimos algumas relações explícitas para a aberração e conceitos relacionados à aberração.

Primeiro encontraremos uma expressão local (numa vizinhança da origem) para uma curva que passa pelo origem e que tem como reta tangente o eixo- $x$.

Seja $\alpha: I \rightarrow \mathbb{R}^{2}$ uma curva com velocidade unitária, pondo $\alpha(s)=(x(s), y(s))$ e com $\alpha(0)=(0,0), \alpha^{\prime}(0)=(1,0)$.

Consideremos a expansão canônica de uma curva numa vizinhança de (0,0). Essa expansão é obtida a partir da expansão da série de Maclaurin da curva no ponto $\mathrm{P}$, em termos de comprimento de arco $s$ ao longo da curva:

$$
\begin{aligned}
& x(s)=a_{0}+\frac{a_{1}}{1 !} s+\frac{a_{2}}{2 !} s^{2}+\frac{a_{3}}{3 !} s^{3}+\ldots \\
& y(s)=b_{0}+\frac{b_{1}}{1 !} s+\frac{b_{2}}{2 !} s^{2}+\frac{b_{3}}{3 !} s^{3}+\ldots
\end{aligned}
$$


$a_{0}=x(0)=0, b_{0}=y(0)=0$,

$a_{1}=\left(\frac{d x}{d s}\right)_{0}=1, b_{1}=\left(\frac{d y}{d s}\right)_{0}=0$

onde o sub-índice 0 indica a estimativa em $s=0$.

Os coeficientes de ordem superior se expressam em termos do raio de curvatura $\rho$ e suas derivadas com respeito a $\psi$, onde $\psi(s)$ é o ângulo formado entre a tangente a $\alpha$ em $s$ e o eixo- $x$.

Denotamos com "'" a derivação com respeito a $\psi$.

Têm-se

$$
\frac{d x}{d s}=\cos \psi \text { e } \frac{d y}{d s}=\operatorname{sen} \psi,(\alpha \text { tem velocidade unitária }) .
$$

Usando o fato

$$
\frac{d \psi}{d s}=\frac{1}{\rho}
$$

segue por diferenciação,

- $\frac{d^{2} \psi}{d s^{2}}=\frac{d}{d s}\left(\frac{1}{\rho}\right)=-\frac{1}{\rho^{2}} \frac{d \rho}{d s}=-\frac{1}{\rho^{2}} \frac{d \rho}{d \psi} \frac{d \psi}{d s}=-\frac{\dot{\rho}}{\rho^{3}}$,

- $\frac{d^{3} \psi}{d s^{3}}=\frac{d}{d s}\left(-\frac{\dot{\rho}}{\rho^{3}}\right)=-\left(\frac{1}{\rho^{3}} \frac{d}{d s} \dot{\rho}+\dot{\rho} \frac{d}{d s} \rho^{-3}\right)=-\frac{\ddot{\rho}}{\rho^{4}}+\frac{3 \dot{\rho}^{2}}{\rho^{5}}$

- $\frac{d^{4} \psi}{d s^{4}}=\frac{10 \rho \dot{\rho} \ddot{\rho}-\rho^{2} \dddot{\rho}-15 \dot{\rho}^{3}}{\rho^{7}}$.

$\frac{d^{2} x}{d s^{2}}=\frac{d}{d s}(\cos \psi)=-\frac{d \psi}{d s} \operatorname{sen} \psi=-\frac{1}{\rho} \operatorname{sen} \psi$,

$\frac{d^{2} y}{d s^{2}}=\frac{d}{d s}(\operatorname{sen} \psi)=\frac{\mathrm{d} \psi}{\mathrm{ds}} \cos \psi=\frac{1}{\rho} \cos \psi$. Então

$$
a_{2}=\left(\frac{d^{2} x}{d s^{2}}\right)_{0}=0 \text { e } b_{2}=\left(\frac{d^{2} y}{d s^{2}}\right)_{0}=\frac{1}{\rho},
$$

$\frac{d^{3} x}{d s^{3}}=\frac{d}{d s}\left(-\frac{d \psi}{d s} \operatorname{sen} \psi\right)=-\frac{\mathrm{d}^{2} \psi}{\mathrm{ds}^{2}} \operatorname{sen} \psi-\left(\frac{\mathrm{d} \psi}{\mathrm{ds}}\right)^{2} \cos \psi=\frac{\dot{\rho}}{\rho^{3}} \operatorname{sen} \psi-\frac{1}{\rho^{2}} \cos \psi$,
$\frac{d^{3} y}{d s^{3}}=\frac{d}{d s}\left(\frac{d \psi}{d s} \cos \psi\right)=\frac{d^{2} \psi}{d s^{2}} \cos \psi-\left(\frac{d \psi}{d s}\right)^{2} \operatorname{sen} \psi=-\frac{\dot{\rho}}{\rho^{3}} \cos \psi-\frac{1}{\rho^{2}} \operatorname{sen} \psi$. Então

$$
a_{3}=\left(\frac{d^{3} x}{d s^{3}}\right)_{0}=-\frac{1}{\rho^{2}} \text { e } b_{3}=\left(\frac{d^{3} y}{d s^{3}}\right)_{0}=-\frac{\dot{\rho}}{\rho^{3}}
$$

$\frac{d^{4} x}{d s^{4}}=\frac{d}{d s}\left(-\frac{d^{2} \psi}{d s} \operatorname{sen} \psi-\left(\frac{\mathrm{d} \psi}{\mathrm{ds}}\right)^{2} \cos \psi\right)=-\frac{\mathrm{d}^{3} \psi}{\mathrm{ds}^{3}} \operatorname{sen} \psi-3 \frac{\mathrm{d}^{2} \psi}{\mathrm{ds}^{2}} \frac{\mathrm{d} \psi}{\mathrm{ds}} \cos \psi+\left(\frac{\mathrm{d} \psi}{\mathrm{ds}}\right)^{3} \operatorname{sen} \psi$ 


$$
\begin{gathered}
=\frac{\rho \ddot{\rho}-3 \dot{\rho}^{2}}{\rho^{5}} \operatorname{sen} \psi+3 \frac{\dot{\rho}}{\rho^{4}} \cos \psi+\frac{1}{\rho^{3}} \operatorname{sen} \psi \\
\frac{d^{4} y}{d s^{4}}=\frac{d}{d s}\left(\frac{d^{2} \psi}{d s} \cos \psi-\left(\frac{d \psi}{d s}\right)^{2} \operatorname{sen} \psi\right)=\frac{\mathrm{d}^{3} \psi}{\mathrm{ds}^{3}} \cos \psi-3 \frac{\mathrm{d}^{2} \psi}{\mathrm{ds}^{2}} \frac{\mathrm{d} \psi}{\mathrm{ds}} \operatorname{sen} \psi-\left(\frac{\mathrm{d} \psi}{\mathrm{ds}}\right)^{3} \cos \psi \\
=-\frac{\rho \ddot{\rho}-3 \dot{\rho}^{2}}{\rho^{5}} \cos \psi+3 \frac{\dot{\rho}}{\rho^{4}} \operatorname{sen} \psi-\frac{1}{\rho^{3}} \cos \psi . \text { Então } \\
a_{4}=\left(\frac{d^{4} x}{d s^{4}}\right)_{0}=\frac{3 \dot{\rho}}{\rho^{4}} \text { e } b_{4}=\left(\frac{d^{4} x}{d s^{4}}\right)_{0}=-\frac{\rho^{2}-3 \dot{\rho}^{2}+\rho \ddot{\rho}}{\rho^{5}} .
\end{gathered}
$$

Substituindo estes coeficientes na serie de Mclaurin, obtem-se a expansão canônica da curva tangente para o eixo- $x$ em $P$.

$$
\begin{gathered}
x(s)=1 s-\frac{1}{6 \rho^{2}} s^{3}+\frac{\dot{\rho}}{8 \rho^{4}} s^{4}+\frac{\rho^{2}-15 \dot{\rho}^{2}+4 \rho \ddot{\rho}}{120 \rho^{6}} s^{5}+\ldots \\
y(s)=\frac{1}{2 \rho} s^{2}-\frac{\dot{\rho}}{6 \rho^{3}} s^{3}-\frac{\rho^{2}-3 \dot{\rho}^{2}+\rho \ddot{\rho}}{24 \rho^{5}} s^{4}+\frac{6 \rho^{2} \dot{\rho}+10 \rho \dot{\rho} \ddot{\rho}-\rho^{2} \dddot{\rho}-15 \dot{\rho}^{3}}{120 \rho^{7}} s^{5}+\ldots
\end{gathered}
$$

Alternativamente, a variável $y$ pode ser expressa diretamente em termos de $x$. Considerando a expansão geral da forma $y=A x^{2}+B x^{3}+C x^{4}+D x^{5}+\ldots$, substituindo da expansão canônica acima obtem-se,

$$
y=\frac{1}{2 \rho} x^{2}-\frac{\dot{\rho}}{6 \rho^{3}} x^{3}+\frac{3 \rho^{2}+3 \dot{\rho}^{2}-\rho \ddot{\rho}}{24 \rho^{5}} x^{4}+\frac{10 \rho \dot{\rho} \ddot{\rho}-19 \rho^{2} \dot{\rho}-\rho^{2} \dddot{\rho}-15 \dot{\rho}^{3}}{120 \rho^{7}} x^{5}+\ldots
$$

\subsubsection{Raio de aberração}

Nesta seção apresentamos relações explícitas para o raio de aberração. O que procuramos nesta seção é relações sensiveis ao raio de aberração. Da equação (4.4) temos uma relação para o raio de aberração.

Das equações (4.4) e (4.3) tem-se

$$
R=\frac{\rho \cos \delta}{1-\dot{\delta}} \quad \text { e } \quad \tan \delta=\frac{\dot{\rho}}{3 \rho}
$$

Por outro lado,

$$
\cos \delta=\frac{1}{\sqrt{1+\tan ^{2} \delta}}=\frac{1}{\sqrt{1+\left(\frac{\dot{\rho}}{3 \rho}\right)^{2}}}=\frac{3 \rho}{\sqrt{9 \rho^{2}+\dot{\rho}^{2}}}=\frac{3 \rho \sqrt{9 \rho^{2}+\dot{\rho}^{2}}}{9 \rho^{2}+\dot{\rho}^{2}}
$$


também temos, $\delta=\arctan \left(\frac{\dot{\rho}}{3 \rho}\right)$ logo por diferenciação,

$\dot{\delta}=\frac{d \delta}{d \psi}=\frac{1}{1+\left(\frac{\dot{\rho}}{3 \rho}\right)^{2}} \frac{d}{d \psi}\left(\frac{\dot{\rho}}{3 \rho}\right)=\frac{9 \rho^{2}}{9 \rho^{2}+\dot{\rho}^{2}}\left(\frac{\ddot{\rho}}{3 \rho}-\frac{\dot{\rho}^{2}}{3 \rho^{2}}\right)=\frac{3 \rho \ddot{\rho}-3 \dot{\rho}^{2}}{9 \rho^{2}+\dot{\rho}^{2}}$.

Substituimos as relações acima na relação para o raio de aberração e obtemos,

$$
R=\frac{3 \rho^{2} \sqrt{9 \rho^{2}+\dot{\rho}^{2}}}{9 \rho^{2}+4 \dot{\rho}^{2}-3 \rho \ddot{\rho}}
$$

Agora uma relação para a aberração,

$\frac{\dot{\rho}}{3 \rho}=\frac{1}{3} \frac{d \rho}{d \psi} \frac{d \psi}{d s}=\frac{1}{3} \frac{d \rho}{d s}=\frac{1}{3} \frac{d}{d s}\left(\frac{1}{k}\right)=-\frac{1}{3 k^{2}} \frac{d k}{d s}=-\frac{1}{3 k^{2}} \frac{d k}{d x} \frac{d x}{d s}=-\frac{1}{3 k^{2}} \frac{d k}{d x} \frac{1}{\left(1+\left(y^{\prime}\right)^{2}\right)^{\frac{1}{2}}}$

por derivação de $k$ na equação (4.2) tem-se,

$$
\frac{d k}{d x}=\frac{y^{\prime \prime \prime}\left(1+\left(y^{\prime}\right)^{2}\right)^{\frac{3}{2}}-3 y^{\prime} y^{\prime \prime}\left(1+\left(y^{\prime}\right)^{2}\right)^{\frac{1}{2}}}{\left(1+\left(y^{\prime}\right)^{2}\right)^{3}}
$$

e substituindo na relação acima tem-se uma relação para a aberração. Além disso,

$$
\tan \delta=\frac{\dot{\rho}}{3 \rho}=y^{\prime}-\frac{1+\left(y^{\prime}\right)^{2}}{3\left(y^{\prime \prime}\right)^{2}} y^{\prime \prime \prime}
$$

Agora considere

$$
\delta=\arctan \left(y^{\prime}-\frac{1+\left(y^{\prime}\right)^{2}}{3\left(y^{\prime \prime}\right)^{2}} y^{\prime \prime \prime}\right),
$$

e fazendo contas análogas como se fez para obter o raio de aberração acima, obtemos a equação cartesiana para o raio de aberração.

$$
R=\frac{3 y^{\prime \prime} \sqrt{\left(y^{\prime \prime \prime}\right)^{2}+\left(y^{\prime} y-3\left(y^{\prime \prime}\right)^{2}\right)^{2}}}{3 y^{\prime \prime} y^{(i v)}-5\left(y^{\prime \prime \prime}\right)^{2}} .
$$

\subsubsection{Centro de aberração}

Em [15] por aplicações de trigonometria encontra-se relações para o centro de aberração $(\xi, \eta)$.

$$
x-\xi=R \sin (\psi-\delta) \quad \eta-y=R \cos (\psi-\delta) .
$$

Para obter equações cartesianas para o centro de aberração consideremos as seguintes relações trigonometricas,

$$
\operatorname{sen} \alpha=\frac{\tan \alpha}{\sqrt{1+\tan ^{2} \alpha}}
$$




$$
\cos \alpha=\frac{1}{\sqrt{1+\tan ^{2} \alpha}}
$$

Considerando as relações trigonometricas acima e as equações (4.1) e (4.7) obtem-se a equação cartesiana para o centro de aberração,

$$
\begin{gathered}
\xi=x-\frac{3 y^{\prime \prime} y^{\prime \prime \prime}}{3 y^{\prime \prime} y^{(i v)}-5\left(y^{\prime \prime \prime}\right)^{2}} \\
\eta=y+\frac{3 y^{\prime \prime}\left(3\left(y^{\prime \prime}\right)^{2}-y^{\prime} y^{\prime \prime \prime}\right)}{3 y^{\prime \prime} y^{(i v)}-5\left(y^{\prime \prime \prime}\right)^{2}}
\end{gathered}
$$

Definição 4.5. O lugar de centros de aberração de uma curva é chamada de curva de aberração.

A curva de aberração é o análogo à evoluta de uma curva, a qual é o lugar dos centros de curvatura da curva dada.

\subsection{Contato de curvas}

Lembremos que, de acordo com a Definição (2.6), duas curvas planas dadas por $y=$ $f(x)$ e $y=g(x)$ tem $n$-ponto de contato em $x=a$ se,

$$
\begin{gathered}
f^{(k)}(a)=g^{(k)}(a) \text { para } k=0,1, \ldots, n-1, \mathrm{e} \\
f^{(n)}(a) \neq g^{(n)}(a),
\end{gathered}
$$

onde as derivadas são em relação de $x$.

Este caso se reduz por translação e rotação ao estudo de duas curvas que tem ponto comum na origem de coordenadas (ver Proposição (1.13)).

Suponhamos que duas curvas tem uma mesma reta tangente num ponto comum. Escolhendo o ponto comum como $(0,0)$ e a tangente comum como $y=0$, pode-se parametrizar a curva localmente por $x$, escrevendo estas curvas como $y=f(x)$ e $y=g(x)$, onde $f$ e $g$ são suaves e $f(0)=g(0)=0$ e $f^{\prime}(0)=g^{\prime}(0)=0$. As curvas acima tem $n$-ponto de contato em $(0,0)$ se,

$$
f^{(k)}(0)=g^{(k)}(0) \text { para } k=2,3, \ldots, n-1 \text { e } f^{(n)}(0) \neq g^{(n)}(0),
$$

onde as derivadas são em relação a $x$. 
Seja $F: \mathbb{R}^{2} \rightarrow \mathbb{R}$ uma curva suave e 0 um valor regular para $F$, então a equação $F(x, y)=0$, define uma curva em $\mathbb{R}^{2}$.

Definição 4.6. O elemento $A_{0} x^{2}+2 B_{0} x y+C_{0} y^{2}+D_{0} x+E_{0} y+F_{0}=0$ da família de cônicas

$$
A x^{2}+2 B x y+C y^{2}+D x+E y+F=0
$$

que tem n-ponto de contato com a curva $F^{-1}(0)$ é chamada uma cônica osculadora para a curva $F^{-1}(0)$ no ponto $P$.

Observação 4.7. Pode existir mais de uma cônica osculadora para uma curva num ponto dado.

\subsection{Ordem do contato}

Nesta seção estudaremos a ordem do contato de uma curva com uma cônica a partir das cônicas osculadoras.

Consideremos uma curva $\nu: I \rightarrow \mathbb{R}^{2}$ parametrizada pelo comprimento de arco. Por conveniencia escolhemos esta curva tangente ao eixo- $x$ na origem. Esta curva pode ser representada pela seguinte expansão canônica (ver equação (4.5)).

$$
y=\frac{1}{2 \rho} x^{2}-\frac{\dot{\rho}}{6 \rho^{3}} x^{3}+\frac{3 \rho^{2}+3 \dot{\rho}^{2}-\rho \ddot{\rho}}{24 \rho^{5}} x^{4}+\ldots
$$

A fim de encontrar cônicas osculantes de ordem três a cinco para esta curva na origem, consideremos a família de cônicas tangentes ao eixo- $x$ na origem,

$$
A x^{2}+2 B x y+C y^{2}+D x+E y+F=0 .
$$

Impondo a condição de que a família seja orientada, já se determina dois dos cinco parâmetros na equação da cônica geral, a saber $F=0$ e $D=0$. Assim a família de cônicas tangentes para o eixo- $x$ na origem é a família a 3-parâmetros,

$$
y=\alpha x^{2}+2 \beta x y+\gamma y^{2} .
$$

Esta família consiste de elipses, parábolas ou hipérboles dependendo do valor do discriminante $\Delta=\alpha \gamma-\beta^{2}$ ser positivo, zero ou negativo, respectivamente.

No caso $\Delta=0$, a família de (4.8) é uma família de parábolas tangentes ao eixo- $x$ na 
origem, e a equação (4.8) reduz-se à família a 2-parâmetros

$$
y=\frac{1}{\alpha}(\alpha x+\beta y)^{2}
$$

Além disso, para o caso da família de círculos tangentes para o eixo- $x$ na origem a equação (4.8) se reduz à família a 1-parâmetro

$$
y=\alpha\left(x^{2}+y^{2}\right)
$$

Usamos a família de círculos (4.10), parábolas (4.9), e cônicas (4.8) para fazer ao menos, 3 -ponto de contato, 4-ponto de contato e 5-ponto de contato com a curva na origem, respectivamente. Daí resultam os chamados círculo-osculador, parábola-osculadora, e cônica osculadora respectivamente.

Primeiro vejamos a expressão local da cônica $y=\alpha x^{2}+2 \beta x y+\gamma y^{2}$, para isto usamos a expansão canônica,

$$
y=\frac{1}{2 \rho} x^{2}-\frac{\dot{\rho}}{6 \rho^{3}} x^{3}+\frac{3 \rho^{2}+3 \dot{\rho}^{2}-\rho \ddot{\rho}}{24 \rho^{5}} x^{4}+\frac{10 \rho \dot{\rho} \ddot{\rho}-19 \rho^{2} \dot{\rho}-\rho^{2} \dddot{\rho}-15 \dot{\rho}^{3}}{120 \rho^{7}} x^{5}+\ldots,
$$

fazendo produto tem-se

$$
y^{2}=\frac{1}{4^{2}} x^{4}-\frac{\dot{\rho}}{6 \rho^{4}} x^{5}+\ldots
$$

e assim,

$$
y=\alpha x^{2}+2 \beta x\left(\frac{1}{2 \rho} x^{2}-\frac{\dot{\rho}}{6 \rho^{3}} x^{3}+\frac{3 \rho^{2}+3 \dot{\rho}^{2}-\rho \ddot{\rho}}{24 \rho^{5}} x^{4}+\ldots\right)+\gamma\left(\frac{1}{4^{\rho} 2} x^{4}-\frac{\dot{\rho}}{6 \rho^{4}} x^{5}+\ldots\right)
$$

portanto,

$$
y=\alpha x^{2}+\frac{\beta}{\rho} x^{3}+\left(\frac{\gamma}{4 \rho^{2}}-\frac{\beta \dot{\rho}}{3 \rho^{3}}\right) x^{4}+\left(\beta \frac{3 \rho^{2}+3 \dot{\rho}^{2}-\rho \ddot{\rho}}{12 \rho^{5}}-\gamma \frac{\dot{\rho}}{6 \rho^{4}}\right) x^{5}+\ldots
$$

Observação 4.8. Comparando as equações (4.11) e (4.12) obtemos informação da ordem de contato da cônica com a curva $\gamma$.

A cônica e a curva $\gamma$ em $t=0$ tem ordem de contato,

1. $\geq 3$ se, e somente se, $\alpha=\frac{1}{2 \rho}$.

2. $\geq 4$ se, e somente se, $\alpha=\frac{1}{2 \rho}$ e $\beta=-\frac{\dot{\rho}}{6 \rho^{2}}$. 
3. $\geq 5$ se, e somente se, $\alpha=\frac{1}{2 \rho}$ e $\quad \beta=-\frac{\dot{\rho}}{6 \rho^{2}} \quad e \quad \gamma=\frac{9 \rho^{2}+5 \dot{\rho}^{2}-3 \rho \ddot{\rho}}{18 \rho^{3}}$.

\subsubsection{Círculo osculador}

Da equação (4.8), uma cônica é um círculo osculador, se $\alpha=\gamma$ e $\beta=0$. Comparando (4.12) com (4.10) obtemos $\alpha=\frac{1}{2 \rho}$, daí (4.10) produz o único círculo osculador

$$
x^{2}+y^{2}-2 \rho y=0
$$

Da observação (4.8), com $\alpha=\frac{1}{2 \rho}$ a família de círculos (4.10) é faz 3-ponto de contato com a curva dada. O raio do círculo osculador é o raio de curvatura $\rho$ e o centro de curvatura é $(0, \rho)$.

\subsubsection{Parábola osculadora}

Da equação (4.8), uma cônica é uma parábola osculador se, $\gamma \alpha=\beta^{2}$. Comparando (4.12) com (4.10) obtemos $\alpha=\frac{1}{2 \rho}$ e $\beta=-\frac{\dot{\rho}}{6 \rho^{2}}$ e $\gamma=\frac{\dot{\rho}^{2}}{18 \rho^{3}}$.

A família de parábolas (4.9) tem 4-ponto de contato com a curva dada. Comparando (4.12) com (4.9) e usando (4.7) tem-se $\alpha=\frac{1}{2 \rho}, \beta=-\frac{\dot{\rho}}{6 \rho^{2}}=-\frac{1}{2 \rho} \tan \delta$.

Substituindo $\alpha$ e $\beta=-\frac{\dot{\rho}}{6 \rho^{2}}$ em (4.9) produz a única parábola osculadora.

$$
(x-\tan \delta)^{2}-2 \rho y=0,
$$

para a curva dada na origem. Seu foco é

$$
\left(-\frac{\rho}{4} \operatorname{sen} 2 \delta, \frac{\rho}{2} \cos ^{2} \delta\right)
$$

sua diretriz é

$$
x \tan \delta+y+\frac{\rho}{2}=0
$$

e seu eixo é

$$
x-y \tan \delta+\frac{\rho}{2} \operatorname{sen} 2 \delta=0
$$

O eixo da parábola osculante é paralelo ao eixo de aberração na origem, a saber $y=$ $x \cot \delta$. Como observado anteriormente, esse fato pode ser usado para dar uma definição alternativa de aberração que não envolve um argumento de limite explícito. 


\subsubsection{Cônica osculadora}

A família de cônicas (4.8) é usada para fazer 5-ponto de contato com a curva dada. Comparando (4.5) com (4.8) se satisfaz

$$
\alpha=\frac{1}{2 \rho}, \quad \beta=-\frac{\dot{\rho}}{6 \rho^{2}}=-\frac{1}{2 \rho} \tan \delta, \quad \gamma=\frac{9 \rho^{2}+5 \dot{\rho}^{2}-3 \rho \ddot{\rho}}{18 \rho^{3}} .
$$

O parâmetro $\gamma$ é determinado em termos de suas derivadas até a ordem 4. Substituindo $\alpha, \beta$ e $\gamma$ em (4.8) fornece uma única cônica osculadora para a curva dada no origem

$$
(3 \rho x-\dot{\rho} y)^{2}+\left(9 \rho^{2}+4 \dot{\rho}^{2}-3 \rho \ddot{\rho}\right) y^{2}-18 \rho^{3} y=0,
$$

ou mais simplesmente

$$
(x-y \tan \delta)^{2}+\sec ^{2} \delta(1-\dot{\delta}) y^{2}-2 \rho y=0 .
$$

O discriminante da cônica é

$$
\Delta=\alpha \gamma-\beta^{2}=\frac{1}{36 \rho^{4}}\left(9 \rho^{2}+4 \dot{\rho}^{2}-3 \rho \ddot{\rho}\right)=\frac{\sec ^{2} \delta}{4 \rho^{2}}(1-\dot{\delta}) .
$$

A cônica é uma elipse, parábola, ou hipérbole dependendo se $\Delta$ seja positivo, zero, ou negativo, respectivamente. Assim um ponto na curva original na qual a cônica é uma elipse, parábola, ou hipérbole é chamado um ponto de curvatura eliptica, parabólica, ou hiperbólica, respectivamente.

O centro da cônica osculante (4.15) é:

$$
\left(\frac{3 \dot{\rho} \rho^{2}}{9 \rho^{2}+4 \dot{\rho}^{2}-3 \rho \ddot{\rho}}, \frac{9 \rho^{3}}{9 \rho^{2}+4 \dot{\rho}^{2}-3 \rho \ddot{\rho}}\right) .
$$

Seu centro pertence ao eixo de aberração

$$
y=\frac{3 \rho}{\dot{\rho}} x,
$$

e em uma distância

$$
R=\frac{3 \rho^{2} \sqrt{9 \rho^{2}+\dot{\rho}^{2}}}{9 \rho^{2}+4 \dot{\rho}^{2}-3 \rho \ddot{\rho}}
$$

desde a origem.

Comparando com (4.6) demonstra-se que o centro da cônica osculante é o centro de aberração. 
Escrevendo o discriminante (4.16) da cônica osculante, na forma cartesiana tem-se

$$
\Delta=\frac{3 y^{\prime \prime} y^{(i v)}-5\left(y^{\prime \prime \prime}\right)^{2}}{36\left(y^{\prime \prime}\right)^{2}\left(1+\left(y^{\prime}\right)^{2}\right)}
$$

Quando um ponto tem curvatura parabólica, a equação (4.18) pode ser escrita na forma

$$
3 y^{\prime \prime} y^{(i v)}-5\left(y^{\prime \prime \prime}\right)^{2}=0
$$

mais compactamente, $\left(\left(y^{\prime \prime}\right)^{-\frac{2}{3}}\right)^{\prime \prime}=0$.

\subsubsection{Contato de ordem 6}

Nesta seção apresentamos uma caracterização, para que uma cônica osculante, faça ao menos 6-ponto de contato com uma curva num ponto dado.

A seguir algumas relações entre as derivadas da curvatura e as derivadas do raio de curvatura.

Por diferenciação de $\rho=\frac{1}{k}$ tem-se,

- $\dot{\rho}=\frac{d \rho}{d \psi}=-\frac{1}{k^{2}} \frac{d k}{d \psi}=-\frac{1}{k^{2}} \frac{d k}{d s} \frac{d s}{d \psi}=-\frac{k^{\prime}}{k^{3}}$,

- $\ddot{\rho}=\frac{d}{d \psi}\left(-\frac{k^{\prime}}{k^{3}}\right)=\frac{3\left(k^{\prime}\right)^{2}-k^{\prime \prime} k}{k^{5}}$

- $\dddot{\rho}=\frac{10 k k^{\prime} k^{\prime \prime}-k^{\prime \prime \prime} k^{2}-15\left(k^{\prime}\right)^{3}}{k^{7}}$

Daí os coeficientes $\alpha, \beta$ e $\gamma$ são dados em função de $k$ pelas seguintes relações,

$$
\alpha=\frac{k}{2}, \beta=\frac{k^{\prime}}{6 k} \text { e } \gamma=\frac{9 k^{4}-5\left(k^{\prime}\right)^{2}+3 k k^{\prime \prime}}{36 k^{2}} .
$$

Assim obtem-se também a relação para o discriminante $\Delta=\alpha \gamma-\beta^{2}$,

$$
\Delta=\frac{9 k^{4}-5\left(k^{\prime}\right)^{2}+3 k k^{\prime \prime}}{36 k^{2}}
$$

A seguir uma relação para o eixo de aberração.

O eixo de aberração tem a forma $y=\frac{3 \rho}{\dot{\rho}} x$, substituindo as relações acima tem-se, $y=-\frac{3 k^{2}}{k^{\prime}} x$ portanto,

$$
((x, y)-(0,0)) \cdot\left(3 k^{2}(1,0)+k^{\prime}(0,1)\right)=0 .
$$


Analogamente podemos conhecer o centro da cônica osculadora em função da curvatura. De fato como,

$$
\left(\frac{3 \dot{\rho} \rho^{2}}{9 \rho^{2}+4 \dot{\rho}^{2}-3 \rho \ddot{\rho}}, \frac{9 \rho^{3}}{9 \rho^{2}+4 \dot{\rho}^{2}-3 \rho \ddot{\rho}}\right) .
$$

Substituindo as relações acima, temos

$$
9 \rho^{2}+4 \dot{\rho}^{2}-3 \rho \ddot{\rho}=\frac{9 k^{4}-5\left(k^{\prime}\right)^{2}+3 k k^{\prime \prime}}{k^{6}},
$$

logo o centro da cônica osculadora é

$$
\left(\frac{-3 k k^{\prime}}{9 k^{4}-5\left(k^{\prime}\right)^{2}+3 k k^{\prime \prime}}, \frac{9 k^{3}}{9 k^{4}-5\left(k^{\prime}\right)^{2}+3 k k^{\prime \prime}}\right)
$$

Agora apresentamos uma relação para que o contato entre uma curva e uma cônica num ponto dado, tenha ordem 6 .

Seja $\nu: I \rightarrow \mathbb{R}^{2}$ uma curva parametrizada por comprimento de arco, com $\nu(0)=(0,0)$ e $\nu^{\prime}(0)=(1,0)$. Da seção anterior sabemos que dita curva tem uma expressão local da forma,

$$
y=\frac{1}{2 \rho} x^{2}-\frac{\dot{\rho}}{6 \rho^{3}} x^{3}+\frac{3 \rho^{2}+3 \dot{\rho}^{2}-\rho \ddot{\rho}}{24 \rho^{5}} x^{4}+\frac{10 \rho \dot{\rho} \ddot{\rho}-19 \rho^{2} \dot{\rho}-\rho^{2} \dddot{\rho}-15 \dot{\rho}^{3}}{120 \rho^{7}} x^{5}+\ldots
$$

Agora consideremos a conica osculadora $y=\alpha x^{2}+2 \beta x y+\gamma y^{2}$ que faz ao menos 5-ponto contato com a curva $\nu$, da seção anterior vimos que os coeficientes tem a forma,

$$
\alpha=\frac{1}{2 \rho}, \quad \beta=-\frac{\dot{\rho}}{6 \rho^{2}}=-\frac{1}{2 \rho} \tan \delta, \quad \gamma=\frac{9 \rho^{2}+5 \dot{\rho}^{2}-3 \rho \ddot{\rho}}{18 \rho^{3}} .
$$

Esta cônica tem a seguinte forma local

$$
y=\frac{1}{2 \rho} x^{2}-\frac{\dot{\rho}}{6 \rho^{3}} x^{3}+\frac{3 \rho^{2}+3 \dot{\rho}^{2}-\rho \ddot{\rho}}{24 \rho^{5}} x^{4}+\frac{-27 \rho^{2} \dot{\rho}-19 \dot{\rho}^{3}+9 \rho \dot{\rho} \ddot{\rho}}{216 \rho^{7}} x^{5}+\ldots .
$$

Se desejamos que a cônica tenha ao menos 6-ponto de contato então deve-se de satisfazer:

$$
\frac{-27 \rho^{2} \dot{\rho}-19 \dot{\rho}^{3}+9 \rho \dot{\rho} \ddot{\rho}}{216 \rho^{7}}=\frac{10 \rho \dot{\rho} \ddot{\rho}-19 \rho^{2} \dot{\rho}-\rho^{2} \dddot{\rho}-15 \dot{\rho}^{3}}{120 \rho^{7}}
$$

desenvolviendo, isto é equivalente a ter,

$$
36 \rho^{2} \dot{\rho}-45 \rho \dot{\rho} \ddot{\rho}+40 \dot{\rho}^{3}+9 \rho^{2} \dddot{\rho}=0
$$


usando as relações:

$\dot{\rho}=-\frac{k^{\prime}}{k^{3}}, \quad \ddot{\rho}=\frac{3\left(k^{\prime}\right)^{2}-k^{\prime \prime} k}{k^{5}}, \quad \dddot{\rho}=\frac{10 k k^{\prime} k^{\prime \prime}-k^{\prime \prime \prime} k^{2}-15\left(k^{\prime}\right)^{3}}{k^{7}}$, obtem-se

$$
36 k^{4} k^{\prime}+40\left(k^{\prime}\right)^{3}-45 k k^{\prime} k^{\prime \prime}+9 k^{2} k^{\prime \prime \prime}=0 .
$$

Esta é a caracterização para que a cônica osculadora tenha ao menos 6-ponto de contato com a curva $\nu$. 


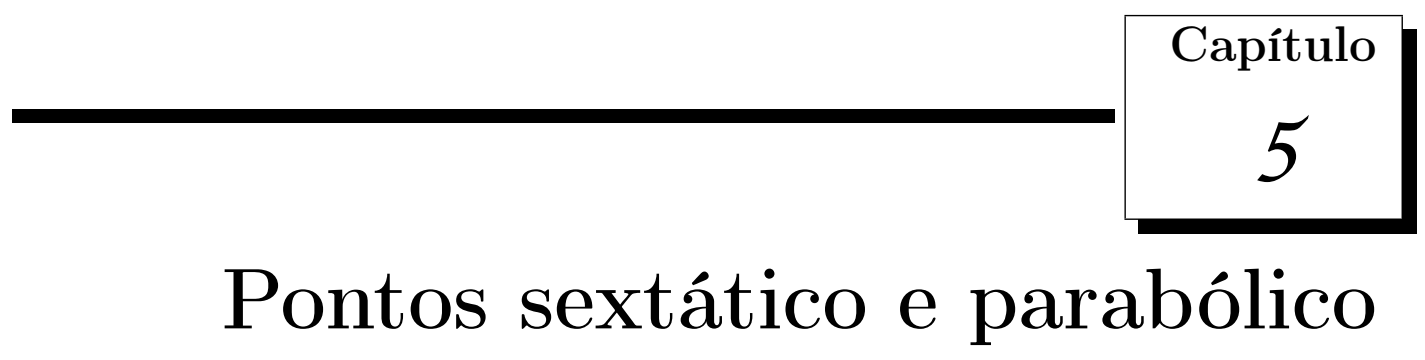

Nesta seção definimos as propriedades genéricas como faz Bruce e Giblin em [3], provamos a existência do pontos sextáticos seguindo o que faz Fidall em [8] e estudamos a geometria da evoluta afim e normal afim, usando teoria de singularidades e o contato entre curvas, isto seguindo [11].

Neste capítulo usamos dois parâmetros, a saber o comprimento de arco e o comprimento de arco afim, e usamos os símbolos, · e ' para fazer referência à diferenciação em relação aos parâmetros comprimento de arco e comprimento de arco afim, respectivamente.

\subsection{Propriedade genérica}

Definição 5.1. Uma propriedade $P$ é dita ser uma propriedade densa, ou que se satisfaz para um conjunto denso de curvas planas quando se tem o seguinte:

Para qualquer curva $\gamma: I \rightarrow \mathbb{R}^{2}$ existe uma vizinhança aberta $U$ de 0 em algum espaço Euclidiano $\mathbb{R}^{N}$ e uma família de curvas planas regulares $\tilde{\gamma}: I \times U \rightarrow \mathbb{R}^{2}$ tal que,

- $\tilde{\gamma}(t, 0)=\gamma(t)$,

- para alguma sequência $\left\{u_{n}\right\}$ em $U$ com $\lim _{n \rightarrow \infty} u_{n}=0$, a propriedade $P$ verifica-se para a sequência de curvas $\gamma_{n}$ definida por $\gamma_{n}(t)=\tilde{\gamma}\left(t, u_{n}\right)$.

Definição 5.2. Uma propriedade $P$ é dita ser uma propriedade aberta, ou que se satisfaz para um conjunto aberto de curvas planas quando para qualquer curva $\gamma: I \rightarrow \mathbb{R}^{2}$ com a propriedade $P$ e qualquer família $\tilde{\gamma}: I \times U \rightarrow \mathbb{R}^{2}$ de curvas $\tilde{\gamma}_{u}$ onde $\tilde{\gamma}_{u}(t)=\tilde{\gamma}(t, u)$, a propriedade $P$ se satisfaz para toda curva $\tilde{\gamma}_{u}$, com u em alguma vizinhança $U_{1}$ de 0 . 
Definição 5.3. Uma propriedade $P$ se diz uma propriedade genérica ou se satisfaz para um conjunto genérico de curvas se, é aberta e densa.

Assim dizer que uma propriedade $\mathrm{P}$ é densa significa que, para cualquer curva que escolhamos pode-se encontrar uma outra curva numa vizinhança desta que satisfaz esta propriedade $\mathrm{P}$.

\subsection{Existência dos pontos sextáticos}

Nesta seção consideramos curvas compactas que são imagens do círculo unitário $\mathbb{S}^{1}$ no lugar de intervalos abertos de $\mathbb{R}$.

Seja $\mathbb{S}^{1} \subset \mathbb{R}^{2}$ um círculo unitário e seja $\theta: \mathbb{R} \rightarrow \mathbb{S}^{1}$, definido por $\theta(s)=(\cos s, \sin s)$. Lembre-se que $\theta$ assim definido é uma imersão $C^{\infty}$ não injetora (para isto ver [13], p. 41).

Definição 5.4. Dizemos que $\gamma: \mathbb{S}^{1} \rightarrow \mathbb{R}^{2}$ é suave se, e somente se, $\gamma \circ \theta: \mathbb{R} \rightarrow \mathbb{R}^{2}$ é suave. De igual forma $\gamma$ é uma curva regular quando $\gamma \circ \theta$ é uma curva regular.

Dando orientação anti-horaria para $\mathbb{S}^{1}$, obtemos uma tangente e normal bem definida em qualquer ponto $\gamma(t)$, com $t \in \mathbb{S}^{1}$.

Se $I$ é um subconjunto aberto de $\mathbb{S}^{1}$, dizemos que $\gamma: I \rightarrow \mathbb{R}^{2}$ é uma curva regular quando para qualquer componente conexa $J$ de $\theta^{-1}(I), \gamma \circ \theta: J \rightarrow \mathbb{R}^{2}$ é uma curva regular.

Uma curva plana $M$, fechada e não degenerada (sem inflexões) é também chamada de oval (em [8]). No que segue desta seção utilizamos este nome. Observemos que, com abuso de linguagem, associamos uma curva $\gamma: \mathbb{S}^{1} \rightarrow \mathbb{R}^{2}$ com sua imagem $M=\gamma\left(\mathbb{S}^{1}\right)$.

A seguir apresentamos propriedades genéricas que serão usadas no que segue do capítulo.

$M_{1}$ : Não existe cônica com mais que 6-ponto de contato com $\mathrm{M}$.

$M_{2}$ : Não existe círculo com mais que 4-ponto de contato com $\mathrm{M}$.

$M_{3}$ : $\mathrm{O}$ número de pontos $p \in \mathbf{M}$ onde a única cônica não singular tocando $\mathbf{M}$ em $p$ com ao menos 5-ponto de contato é uma parábola é finito.

$M_{4}$ : Não exíste parábola que tem 6-ponto de contato com $\mathrm{M}$.

Nos trabalhos de Fidall ([8],[9]), Izumiya [11], entre outros são usadas estas propriedades genéricas. 
Definição 5.5. Seja $\gamma: \mathbb{S}^{1} \rightarrow \mathbb{R}^{2}$ uma oval.

1. Dizemos que um ponto $p_{0}=\gamma\left(t_{0}\right)$ é um ponto sextático se existe uma cônica tocando $\gamma(I)$ em $p_{0}$ com ao menos 6-ponto de contato.

2. Dizemos que um ponto $p_{0}=\gamma\left(t_{0}\right)$ é um ponto parabólico se existe uma única parabóla tocando $\gamma(I)$ em $p_{0}$ com 5-ponto de contato.

Teorema 5.6. Seja $\gamma: I \rightarrow \mathbb{R}^{2}$ uma curva regular, sem inflexões e parametrizada por comprimento de arco. Então $p_{0}=\gamma\left(t_{0}\right)$ é um ponto sextático se, e somente se,

$$
36 k\left(t_{0}\right)^{4} \dot{k}\left(t_{0}\right)+40 \dot{k}\left(t_{0}\right)^{3}-45 k\left(t_{0}\right) \dot{k}\left(t_{0}\right) \ddot{k}\left(t_{0}\right)+9 k\left(t_{0}\right)^{2} \dddot{k}\left(t_{0}\right)=0 .
$$

Demonstração: Decorre da equação (4.23) do Capítulo 4.

Seja $\gamma: \mathbb{S}^{1} \rightarrow \mathbb{R}^{2}$ uma curva com velocidade unitária com imagem $M=\gamma\left(\mathbb{S}^{1}\right)$.

Denotemos a única cônica não singular tendo ponto de contato $\geq 5 \operatorname{com~} M$ em $\gamma(t)$ por $C_{t}$, e o centro de $C_{t}$ por $c_{t}$.

Em qualquer $\gamma(t)$, os centros das cônicas tendo contato maior que 4 com $M$ em $\gamma(t)$ pertencem a uma reta que passa por $\gamma(t)$. Da seção anterior sabemos que tal reta é o eixo de aberração, o qual denotamos por $A_{t}$ (ver Equação (4.21)).

Da Equação (4.21) e por rotação e translação obtemos o eixo de aberração,

$$
A_{t}=\left\{x \in \mathbb{S}^{1}:(x-\gamma(t)) \cdot\left(3 k^{2}(t) T(t)+\dot{k}(t) N(t)\right)=0\right\}
$$

onde $T(t)$ e $N(t)$ são os vetores tangente e normal a $M$ em $\gamma(t)$ e $k(t)$ é a curvatura de $M$ em $\gamma(t)$. Agora definimos a função $F$ como,

$$
\begin{aligned}
F: \mathbb{S}^{1} \times \mathbb{R}^{2} \rightarrow \mathbb{R} \\
(t, x) \mapsto(x-\gamma(t)) \cdot\left(3 k^{2}(t) T(t)+\dot{k}(t) N(t)\right) .
\end{aligned}
$$

Então $F^{-1}(0) \cap\left\{(t, x) / x \in \mathbb{R}^{2}\right\}=A_{t}$.

Observe que $x \in A_{t}$ se, e somente se,

$$
x=\gamma(t)+\lambda_{t}\left(-\dot{k}(t) T(t)+3 k^{2}(t) N(t)\right)
$$

para algum $\lambda_{t} \in \mathbb{R}$. 
Teorema 5.7. Seja M uma oval com velocidade unitária satisfazendo as condições $\left(M_{1}\right)$ $\left(M_{4}\right)$. Então,

1. O lugar geométrico de centros $c_{t}$ das cônicas osculadoras de $M$ é exatamente o conjunto discriminante de $F$.

$A \operatorname{sim} c_{t}=\gamma(t)+\lambda_{t}\left(-\dot{k}(t) T(t)+3 k^{2}(t) N(t)\right)$, onde

$$
\lambda_{t}=\frac{3 k(t)}{9 k^{4}(t)+3 k(t) \ddot{k}(t)-5 \dot{k}(t)^{2}} .
$$

2. Supondo que $x$ é finito, $F(-, x)$ tem uma $A_{2}$ singularidade em $t$ se, e somente se, $\gamma(t)$ é um ponto sextático de $M$.

Demonstração: Para o item 1, vejamos primeiro o conjunto discriminante de F.

$$
\mathcal{D}_{F}=\left\{x \in \mathbb{R}^{2}: \exists t \in \mathbb{S}^{1} \operatorname{com} F(t, x)=\frac{\partial F}{\partial t}(t, x)=0\right\} .
$$

$F(t, x)=0$ se, e somente se, $\exists \lambda_{t} \in \mathbb{R}$ tal que

$$
x-\gamma(t)=\lambda_{t}\left(-\dot{k}(t) T(t)+3 k^{2}(t) N(t)\right) .
$$

$$
\begin{aligned}
\frac{\partial F}{\partial t}(t, x) & =\left(-\dot{\gamma}(t)\left(3 k^{2}(t) T(t)+\dot{k}(t) N(t)\right)+(x-\gamma(t)) \cdot\left(6 k(t) \dot{k}(t) T(t)+3 k^{2} \dot{T}(t)+\right.\right. \\
& +\ddot{k}(t) N(T)+\dot{k}(t) \dot{N}(t))) .
\end{aligned}
$$

Pelas equações de Frenet-Serret obtemos,

$\frac{\partial F}{\partial t}(t, x)=-3 k^{2}(t)+(x-\gamma(t)) \cdot\left(5 k(t) \dot{k}(t) T(t)+\left(3 k^{3}+\ddot{k}(t)\right) N(t)\right)$,

$\operatorname{logo} \frac{\partial F}{\partial t}(t, x)=0$ se, e somente se,

$$
-3 k^{2}(t)+(x-\gamma(t)) \cdot\left(5 k(t) \dot{k}(t) T(t)+3 k^{3} \ddot{k}(t) N(t)\right)=0 .
$$

Substituindo a equação (5.1) em (5.2), obtemos

$$
\lambda_{t}=\frac{3 k(t)}{9 k^{4}(t)+3 k(t) \ddot{k}(t)-5 \dot{k}(t)^{2}} .
$$

Portanto $x \in \mathcal{D}_{F}$ se, e somente se,

$$
x=\gamma(t)+\frac{3 k(t)}{9 k^{4}(t)+3 k(t) \ddot{k}(t)-5 \dot{k}(t)^{2}}\left(-\dot{k}(t) T(t)+3 k^{2}(t) N(t)\right),
$$


logo da equação (4.22) e por translação e rotação, tem-se $x=c_{t}$.

Agora vejamos o item 2. Suponhamos $x$ é finito. Para simplificar as contas omitiremos o parâmetro $t$, e denotaremos com $F_{x}=F(-, x)$. Logo $F_{x}=(x-\gamma) \cdot\left(3 k^{2} T+\dot{k} N\right)$ por diferenciação

$$
\begin{aligned}
& \dot{F}_{x}=-3 k^{2}+(x-\gamma) \cdot\left(5 k \dot{k} T+\left(3 k^{3}+\ddot{k}\right) N\right), \mathrm{e} \\
& \ddot{F}_{x}=-11 k \dot{k}+(x-\gamma) \cdot\left(\left(-3 k^{4}+4 k \ddot{k}+5 \dot{k}^{2}\right) T+\left(14 k^{2} \dot{k}+\dddot{k}\right) N\right) .
\end{aligned}
$$

Pelo item $1, F_{x}(t)=\dot{F}_{x}(t)=0$ se, e somente se,

$$
x-\gamma(t)=\lambda_{t}\left(-\dot{k}(t) T(t)+3 k^{2}(t) N(t)\right) \text { e, } \lambda_{t}=\frac{3 k(t)}{9 k^{4}(t)+3 k(t) \ddot{k}(t)-5 \dot{k}(t)^{2}} .
$$

Agora adicionando a condição $\ddot{F}_{x}(t)=0$, tem-se

$$
0=-11 k \dot{k}+\frac{3 k}{9 k^{4}+3 k \ddot{k}-5 \dot{k}^{2}}\left(-\dot{k} T+3 k^{2} N\right) \cdot\left(\left(-3 k^{4}+4 k \ddot{k}+5 \dot{k}^{2}\right) T+\left(14 k^{2} \dot{k}+\dddot{k}\right) N\right) .
$$

Desenvolvendo esta relação obtemos

$$
0=\frac{36 k^{4} \dot{k}-45 k \dot{k} \ddot{k}+40 \dot{k}^{3}+9 k^{2} \dddot{k}}{9 k^{4}+3 k \ddot{k}-5 \dot{k}^{2}} .
$$

Como $x$ é finito, $9 k^{4}+3 k \ddot{k}-5 \dot{k}^{2} \neq 0$. Logo esta condição é equivalente a

$$
36 k^{4} \dot{k}-45 k \dot{k} \ddot{k}+40 \dot{k}^{3}+9 k^{2} \dddot{k}=0 .
$$

Pelas condições genéricas $\left(M_{1}\right)-\left(M_{4}\right), F_{x}$ tem tipo $A_{\leq 2}$, logo $F_{x}$ tem singularidade $A_{2}$ se, e somente se,

$$
x-\gamma=\lambda_{t}\left(-\dot{k} T+3 k^{2} N\right), \lambda_{t}=\frac{3 k}{9 k^{4}+3 k \ddot{k}-5 \dot{k}^{2}} .
$$

e

$$
36 k^{4} \dot{k}-45 k \dot{k} \ddot{k}+40 \dot{k}^{3}+9 k^{2} \dddot{k}=0 \text {. }
$$

Isto é, $x$ é centro da cônica osculante e a cônica osculante tem 6-ponto de contato com a curva $\gamma$.

Teorema 5.8. Seja $M$ uma oval, satisfazendo as condições genéricas $M_{3}$ e $M_{4}$. Então existe um ponto sextático em $M$.

Demonstração: Por $M_{3}$ e $M_{4}$ existe um número finito de parábolas quintáticas, ou 
seja parábolas que tem 5 -ponto de contato com $M$, a saber $\gamma\left(t_{1}\right), \ldots, \gamma\left(t_{n}\right)$, definimos a seguinte função:

$$
\begin{gathered}
A: \mathbb{S}^{1}-\left\{t_{1}, \ldots, t_{n}\right\} \rightarrow \mathbb{R} \\
t \mapsto 3 \lambda_{t}(k(t))^{\frac{5}{3}},
\end{gathered}
$$

onde

$$
\lambda_{t}=\frac{3 k(t)}{9 k^{4}(t)+3 k(t) k^{\prime \prime}(t)-5\left(k^{\prime}(t)\right)^{2}} .
$$

Caso 1 Se não existem parábolas quintáticas, isto é se $n=0$, então $A$ está definido para todo $\mathbb{S}^{1}$. Daí existe ponto de máximo para a função $A$, isto é, existe $t \in \mathbb{S}^{1}$ tal que $A(t)$ é um máximo local e portanto $\frac{\partial A}{\partial t}(t)=0$.

Caso 2 Agora suponhamos que $n \geq 1$, então $\mathbb{S}^{1}-\left\{t_{1}, \ldots, t_{n}\right\}$ é particionado num número finito de intervalos abertos. Escolhemos um desses intervalos, chamamos de $I$, sem perda de generalidade, suponhamos que $\bar{I}-I=\left\{t_{1}, t_{2}\right\}$.

$A(t) \neq 0, \forall t \in I$, pois se $A(t)=0$ implica $\lambda_{t}=0 \operatorname{logo}$ como $c_{t} \in A_{t}$ então $c_{t}=\gamma(t)$ portanto, existe uma cônica $C_{t}$, com $c_{t} \in C_{t}$ o qual é um absurdo.

Daí, por continuidade, como $A(t) \neq 0$ para todo $t \in I, A(t)$ tem o mesmo sinal para todo $t \in I$.

Como $t_{1}$ e $t_{2}$ são pontos parabólicos quintáticos $\lim _{t \rightarrow t_{i}}|A(t)|=\infty, i=1,2$, portanto existe um sub-intervalo fechado $\left[s_{1}, s_{2}\right]$ de $I$ tal que $A\left(s_{1}\right)=A\left(s_{2}\right)$. Aplicando o Teorema de Rolle para $A$ no intervalo $J$, existe $t \in\left(s_{1}, s_{2}\right)$ tal que $\frac{\partial A}{\partial t}(t)=0$.

Por outro lado,

$\frac{\partial A}{\partial t}(t)=\frac{\left(3(k(t))^{\frac{5}{3}}\right)}{9 k(t)^{4}+3 k(t) k^{\prime \prime}(t)-5 k^{\prime}(t)^{2}} \cdot\left(36 k(t)^{4} \dot{k}(t)+40 \dot{k}(t)^{3}-45 k(t) \dot{k}(t) \ddot{k}(t)+9 k(t)^{2} \ddot{k}(t)\right)$.

Agora desde que existe $t \in \mathbb{S}^{1}$ tal que $\frac{\partial A}{\partial t}(t)=0$, então existe $t \in \mathbb{S}^{1}$ tal que

$$
36 k(t)^{4} \dot{k}(t)+40 \dot{k}(t)^{3}-45 k(t) \dot{k}(t) \ddot{k}(t)+9 k(t)^{2} \ddot{k}(t)=0,
$$

portanto $\gamma(t)$ é um ponto sextático.

\subsection{Caracterização dos pontos sextáticos e parabólicos}

Proposição 5.9. Seja $\gamma: \mathbb{S}^{1} \rightarrow \mathbb{R}^{2}$ uma curva suave sem pontos de inflexão que satisfaz as condições $M_{1}$ e $M_{3}$. Então temos: 
(i) $p=\gamma\left(s_{0}\right)$ é um ponto parabólico de $\gamma\left(\mathbb{S}^{1}\right)$ se, e somente se, $k_{a}\left(s_{0}\right)=0$.

(ii) $p=\gamma\left(s_{0}\right)$ é um ponto sextático de $\gamma\left(\mathbb{S}^{1}\right)$ se, e somente se, $k_{a}^{\prime}\left(s_{0}\right)=0$.

Demonstração: Para o item (i) consideremos a equação (4.20). Logo $p=\gamma\left(s_{0}\right)$ é um ponto parabólico de $\gamma\left(\mathbb{S}^{1}\right)$ se, e somente se,

$$
9 k^{4}(t)+3 k(t) \ddot{k}(t)-5 \dot{k}(t)^{2}=0 .
$$

Por outro lado, pelo Teorema (3.45) tem-se

$$
9 k_{a}(s) k(t)^{\frac{8}{3}}=9 k^{4}(t)-5 \dot{k}(t)^{2}+3 k(t) \ddot{k}(t) .
$$

Portanto $p=\gamma\left(s_{0}\right)$ é um ponto parabólico de $\gamma\left(\mathbb{S}^{1}\right)$ se, e somente se, $k_{a}\left(s_{0}\right)=0$.

Para o item (ii) usamos o Teorema (3.45), daí

$$
k_{a}(s)=k(t)^{\frac{4}{3}}-\frac{5}{9} k(t)^{-\frac{8}{3}} \dot{k}(t)^{2}+\frac{1}{3} k(t)^{-\frac{5}{3}} \ddot{k}(t) .
$$

Por diferenciação tem-se,

$$
\begin{aligned}
& k_{a}^{\prime}(s)=\frac{4}{3} k(t)^{\frac{1}{3}} \dot{k}(t) \dot{\xi}(s)-\frac{5}{9}\left\{-\frac{8}{3} k(t)^{-\frac{11}{3}} \dot{k}(t)^{3} \dot{\xi}(s)+2 k(t)^{-\frac{8}{3}} \dot{k}(t) \ddot{k}(t) \dot{\xi}(s)\right\}+ \\
&+\frac{1}{3}\left\{-\frac{5}{3} k(t)^{-\frac{8}{3}} \dot{k}(t) \ddot{k}(t) \dot{\xi}(s)+k(t)^{-\frac{5}{3}} \dddot{k}(t) \dot{\xi}(s)\right\}, \\
& k_{a}^{\prime}(s)=\frac{4}{3} \dot{k}(t)+\frac{40}{27} k(t)^{-4} \dot{k}(t)^{3}-\frac{10}{9} k(t)^{-3} \dot{k}(t) \ddot{k}(t)-\frac{5}{9} k(t)^{-3} \dot{k}(t) \ddot{k}(t)+\frac{1}{3} k(t)^{-2} \dddot{k}(t), \\
& 27 k(t)^{4} k_{a}^{\prime}(s)=36 \dot{k}(t) k(t)^{4}+40 \dot{k}(t)^{3}-45 k(t) \dot{k}(t) \ddot{k}(t)+9 k(t)^{2} \dddot{k}(t) .
\end{aligned}
$$

Portanto,

$$
k_{a}^{\prime}\left(s_{0}\right)=0 \Leftrightarrow 36 \dot{k}\left(t_{0}\right) k\left(t_{0}\right)^{4}+40 \dot{k}\left(t_{0}\right)^{3}-45 k\left(t_{0}\right) \dot{k}\left(t_{0}\right) \ddot{k}\left(t_{0}\right)+9 k\left(t_{0}\right)^{2} \dddot{k}\left(t_{0}\right)=0 .
$$

Do Teorema (5.6), $\gamma\left(t_{0}\right)$ é ponto sextático se, e somente se, $k_{a}^{\prime}\left(s_{0}\right)=0$.

Observação 5.10. Da Definição (3.41), $\gamma$ tem inflexão afim em $\gamma\left(s_{0}\right)$ se $k_{a}\left(s_{0}\right)=0$, isto é $\gamma\left(s_{0}\right)$ é um ponto parabólico. Analogamente da Definição (3.42), $\gamma$ tem vértice afim em $\gamma\left(s_{0}\right)$ se $k_{a}^{\prime}\left(s_{0}\right)=0$, isto é, $\gamma\left(s_{0}\right)$ é um ponto sextático. 


\subsection{Classificação de curvas planas}

Teorema 5.11. Seja $\gamma: I \rightarrow \mathbb{R}^{2}$ uma curva sem pontos de inflexão satisfazendo $M_{1}, M_{3}$ e $M_{4}$.

1. Seja $p$ um ponto da evoluta afim de $\gamma$ em $s_{0}$. Então, localmente em $p$, a evoluta afim é:

(a) difeomorfa a uma reta em $\mathbb{R}^{2}$ se $\gamma\left(s_{0}\right)$ não é um ponto sextático,

(b) difeomorfa a uma cúspide em $\mathbb{R}^{2}$ se $\gamma\left(s_{0}\right)$ é um ponto sextático.

2. Seja $p=\gamma^{\prime \prime}\left(s_{0}\right)$ um ponto da normal afim de $\gamma$ em $s_{0}$. Então, localmente em $p$, a curva normal afim é:

(a) difeomorfa a uma reta em $\mathbb{R}^{2}$ se $\gamma\left(s_{0}\right)$ não é um ponto parabólico de $\gamma$,

(b) difeomorfa a uma cúspide em $\mathbb{R}^{2}$ se $\gamma\left(s_{0}\right)$ é um ponto parabólico de $\gamma$.

Demonstração: Como $\gamma$ não tem pontos de inflexão, sem perda de generalidade esta se considera parametrizada pelo comprimento de arco afim.

(1): Consideremos a função distância ao cubo afim sobre $\gamma, F: \mathbb{R} \times \mathbb{R}^{2},\left(s_{0}, x_{0}\right) \rightarrow \mathbb{R}$,

$$
F(s, x)=\left|\gamma^{\prime}(s), \gamma(s)-x\right|
$$

Primeiro descrevemos o conjunto bifurcação $\mathcal{B}_{F}$ de $F$,

$$
\mathcal{B}_{F}=\left\{x \in \mathbb{R}^{2}: \exists t \text { tal que } \frac{\partial F}{\partial s}=\frac{\partial^{2} F}{\partial s^{2}}=0 \text { em }(t, x)\right\}
$$

Se $x \in \mathcal{B}_{F}$, então existe $s_{0} \in \mathbb{R}$, tal que:

$$
\frac{\partial F}{\partial s}\left(s_{0}, x\right)=0 \quad e \frac{\partial^{2} F}{\partial s^{2}}\left(s_{0}, x\right)=0
$$

Por outro lado

$$
\begin{gathered}
F_{x}^{\prime}\left(s_{0}\right)=\frac{\partial F_{x}}{\partial s}\left(s_{0}\right)=\frac{\partial F}{\partial s}\left(s_{0}, x\right) \\
F_{x}^{\prime \prime}\left(s_{0}\right)=\frac{\partial^{2} F_{x}}{\partial s^{2}}\left(s_{0}\right)=\frac{\partial^{2} F}{\partial s^{2}}\left(s_{0}, x\right)
\end{gathered}
$$

Da Proposição (3.59), $x \in \mathcal{B}_{F}$ se, e somente se, $x$ é um ponto da evoluta afim. Isto é 
$k_{a}\left(s_{0}\right) \neq 0$ e $x=\gamma\left(s_{0}\right)+\frac{1}{k\left(s_{0}\right)} \gamma^{\prime \prime}\left(s_{0}\right)$.

Escrevendo $\gamma(s)=\left(x_{1}(s), x_{2}(s)\right)$ e $x=\left(x_{1}, x_{2}\right)$ tem-se

$$
F(s, x)=x_{1}^{\prime}(s) x_{2}(s)-x_{1}^{\prime}(s) x_{2}-x_{2}^{\prime}(s) x_{1}(s)+x_{2}^{\prime}(s) x_{1}
$$

Daí por diferenciação,

$$
\frac{\partial}{\partial x_{1}} F\left(s, x_{1}, x_{2}\right)=x_{2}^{\prime}(s) \text { e } \frac{\partial}{\partial x_{2}} F\left(s, x_{1}, x_{2}\right)=-x_{1}^{\prime}(s)
$$

Por outro lado,

$$
\begin{aligned}
& \bullet j^{1}\left(\frac{\partial F}{\partial x_{i}}\left(s, x_{0}\right)\right)\left(s_{0}\right)=\alpha_{1, i} s, \text { onde } \alpha_{1, i}=\frac{\partial}{\partial s}\left(\frac{\partial F}{\partial x_{i}}\left(s_{0}, x_{0}\right)\right),(i=1,2) \\
& \log \alpha_{1,1}=x_{2}^{\prime \prime}\left(s_{0}\right) \text { e } \alpha_{1,2}=-x_{1}^{\prime \prime}\left(s_{0}\right) \\
& \bullet j^{2}\left(\frac{\partial F}{\partial x_{i}}\left(s, x_{0}\right)\right)\left(s_{0}\right)=\alpha_{1, i} s+\alpha_{2, i} s^{2}, \text { onde } \alpha_{1, i}=\frac{\partial}{\partial s}\left(\frac{\partial F}{\partial x_{i}}\left(s_{0}, x_{0}\right)\right) \mathrm{e} \\
& \alpha_{2, i}=\frac{1}{2} \frac{\partial^{2}}{\partial s^{2}}\left(\frac{\partial F}{\partial x_{i}}\left(s_{0}, x_{0}\right)\right),(i=1,2) \\
& \log , \alpha_{1,1}=x_{2}^{\prime \prime}\left(s_{0}\right), \alpha_{1,2}=-x_{1}^{\prime \prime}\left(s_{0}\right), \alpha_{2,1}=\frac{1}{2} x_{2}^{\prime \prime}\left(s_{0}\right), \alpha_{2,2}=-\frac{1}{2} x_{1}^{\prime \prime}\left(s_{0}\right)
\end{aligned}
$$

Agora vejamos as demonstrações dos itens (a) e (b) do item (1) do teorema.

(a) Suponhamos que $x_{0}$ é um ponto da evoluta afim de $\gamma$ em $s_{0}$ e $\gamma\left(s_{0}\right)$ não é um ponto sextático.

Como $x_{0}=\gamma\left(s_{0}\right)$ não é um ponto sextático, da Proposição (5.9) temos $k_{a}^{\prime}\left(s_{0}\right) \neq 0$; além disso $x_{0} \in \mathcal{B}_{F}$ pois $x_{0}$ é um ponto da evoluta afim de $\gamma$ em $s_{0}$.

Para obter nosso resultado verificamos as condições da Proposição (2.29) isto é, verificamos que $F$ é um desdobramento (p)versal a 2-parâmetros de $f=F_{x_{0}}$ e que $f$ tem singularidade do tipo $A_{2}$ em $s_{0}$.

Afirmação 1. $f: \mathbb{R}, s_{0} \rightarrow \mathbb{R}$ definida como $f(s)=F\left(s, x_{0}\right)$ tem singularidade de tipo $A_{2}$ em $s_{0}$.

De fato, observemos que $f=F_{x_{0}}$, como $x_{0}$ é um ponto da evoluta afim em $s_{0}$ logo da proposição (3.59) $f^{\prime}\left(s_{0}\right)=f^{\prime \prime}\left(s_{0}\right)=0$ além disso, de $k_{a}^{\prime}\left(s_{0}\right) \neq 0$ e da proposição (3.59) tem-se que $f^{\left({ }^{\prime \prime \prime}\right)}\left(s_{0}\right) \neq 0$, logo $f$ tem singularidade do tipo $A_{2}$ em $s_{0}$.

Afirmação 2. F é um desdobramento (p)versal a 2-parâmetros de $f$. 
Para isto, usamos o Teorema (2.20) isto é, a matriz $1 \times 2$ dos coeficientes $\alpha$ tem posto 1 , onde os coeficientes de $\alpha$ vêm de

$$
j^{1}\left(\frac{\partial F}{\partial x_{i}}\left(s, x_{0}\right)\right)\left(s_{0}\right)=\alpha_{1, i} s, i=1,2
$$

assim basta verificar que a matriz $\left(x_{2}^{\prime \prime}\left(s_{0}\right)-x_{1}^{\prime \prime}\left(s_{0}\right)\right)$ tem posto 1 e isto decorre de $\left|\gamma^{\prime}\left(s_{0}\right), \gamma^{\prime \prime}\left(s_{0}\right)\right|=1$.

(b) Suponhamos que $x_{0}$ é um ponto da evoluta afim de $\gamma$ em $s_{0}$ e $x_{0}=\gamma\left(s_{0}\right)$ é um ponto sextático.

Como $x_{0}$ é um ponto sextático da Proposição (5.9), $k_{a}^{\prime}\left(s_{0}\right)=0$ e $x_{0} \in \mathcal{B}_{F}\left(x_{0}\right.$ pertence à evoluta afim).

Igual que acima, para obter nosso resultado verificamos as condições da Proposição (2.29), isto é, verificamos que $F$ é um desdobramento (p)versal 2-parâmetros de $f=F_{x_{0}}$ e $f$ tem singularidade do tipo $A_{3}$ em $s_{0}$.

Afirmação 3. $f: \mathbb{R}, s_{0} \rightarrow \mathbb{R}$ definida como $f(s)=F\left(s, x_{0}\right)$ tem singularidade de tipo $A_{3}$ em $s_{0}$.

De fato, $f=F_{x_{0}}$, como $x_{0}$ é um ponto da evoluta afim em $s_{0}$ logo pela proposição (3.59) $f^{\prime}\left(s_{0}\right)=f^{\prime \prime}\left(s_{0}\right)=0$, além disso de $k_{a}^{\prime}\left(s_{0}\right)=0$ e da proposição $(3.59), f^{\left({ }^{\prime \prime \prime}\right)}\left(s_{0}\right)=0$, logo $f$ tem singularidade do tipo $A_{\geq 3}$ em $s_{0}$ e pelas condições de genericidade $\left(M_{1}\right),\left(M_{3}\right)$ e $\left(M_{4}\right), f$ tem exatamente singularidade do tipo $A_{3}$ em $s_{0}$.

Afirmação 4. F é um desdobramento (p)versal a 2-parâmetros de $f$.

Usamos o Teorema (2.20) é dizer, a matriz $2 \times 2$ dos coeficientes $\alpha$ tem posto 2 onde, os coeficientes de $\alpha$ vêm de

$$
j^{2}\left(\frac{\partial F}{\partial x_{i}}\left(s, x_{0}\right)\right)\left(s_{0}\right)=\alpha_{1, i} s+\alpha_{2, i} s^{2}, i=1,2
$$

assim verificamos que a matriz $\left(\begin{array}{cc}x_{2}^{\prime \prime}\left(s_{0}\right) & -x_{1}^{\prime \prime}\left(s_{0}\right) \\ \frac{1}{2} x_{2}^{\prime \prime \prime}\left(s_{0}\right) & -\frac{1}{2} x_{1}^{\prime \prime \prime}\left(s_{0}\right)\end{array}\right)$ tem posto 2. e isto segue pois, seu determinante é

$$
\frac{1}{2}\left|\gamma^{\prime \prime}\left(s_{0}\right), \gamma^{\prime \prime \prime}\left(s_{0}\right)\right|=\frac{1}{2} k_{a}\left(s_{0}\right) \neq 0 \text {. }
$$

(2): Consideremos a função $\widetilde{H}: I \times \mathbb{S}^{1} \times \mathbb{R} \rightarrow \mathbb{R}$ definida por

$$
\widetilde{H}(s, u, y)=H(s, u)-y
$$


onde $H$ é a função altura afim sobre $\gamma, H(s, u)=\left|\gamma^{\prime}(s), u\right|$, é dizer

$$
\widetilde{H}(s, u, y)=x_{1}^{\prime}(s) u_{2}-x_{2}^{\prime}(s) u_{1}-y
$$

onde $\gamma(s)=\left(x_{1}(s), x_{2}(s)\right), u=\left(u_{1}, u_{2}\right) \in \mathbb{S}^{1}$

Primeiro descrevemos o conjunto discriminante $\mathcal{D}_{\widetilde{H}}$ de $\widetilde{H}$

$$
\mathcal{D}_{\widetilde{H}}=\left\{(u, y) \in \mathbb{S}^{1} \times \mathbb{R}: \text { existe } s \in \mathbb{R} \text { com } \widetilde{H}=\frac{\partial \widetilde{H}}{\partial s}=0 \text { em }(s, u, y)\right\}
$$

Seja $(u, y) \in \mathcal{D}_{\widetilde{H}}$ então,

- $H_{u}^{\prime}(s)=\frac{\partial H}{\partial s}(s, u)=\frac{\partial \widetilde{H}}{\partial s}(s, u, y)=0$.

Da Proposição (3.64), $H_{u}^{\prime}(s)=0$ se, e somente se, $\exists \lambda(s) \in \mathbb{R}$ tal que $u=\lambda(s) \gamma^{\prime \prime}(s)$ então

$$
\begin{gathered}
u_{1}=\lambda(s) x_{1}^{\prime \prime}(s) \text { e } u_{2}=\lambda x_{2}^{\prime \prime}(s) \\
1=u_{1}^{2}+u_{2}^{2}=\lambda(s)^{2}\left(x_{1}^{\prime \prime}(s)^{2}+x_{2}^{\prime \prime}(s)^{2}\right) \text { daí } \lambda(s)= \pm \frac{1}{\sqrt{x_{1}^{\prime \prime}(s)^{2}+x_{2}^{\prime \prime}(s)^{2}}}\left(\gamma^{\prime \prime}(s) \neq 0 .\right)
\end{gathered}
$$

- $\widetilde{H}(s, u, y)=0$ se, e somente se,

$$
y=x_{1}^{\prime}(s) u_{2}-x_{2}^{\prime}(s) u_{1}=x_{1}^{\prime}(s) \lambda(s) x_{2}^{\prime \prime}(s)-x_{2}^{\prime}(s) \lambda(s) x_{1}^{\prime \prime}(s)
$$

então $y=\lambda(s)\left|\gamma^{\prime}(s), \gamma^{\prime \prime}(s)\right|=\lambda(s)$, portanto $y=\lambda(s)$.

Portanto, o conjunto discriminante é

$$
\mathcal{D}^{ \pm}{ }_{\widetilde{H}}=\left\{\left(\lambda(s) \gamma^{\prime \prime}(s), \lambda(s)\right): \lambda(s)= \pm \frac{1}{\sqrt{x_{1}^{\prime \prime}(s)^{2}+x_{2}^{\prime \prime}(s)^{2}}}\right\}
$$

consideremos somente o conjunto $\mathcal{D}_{\widetilde{H}}^{+}$.

Definimos a função $\Phi: \mathbb{R}^{2}-\{0\} \rightarrow \mathbb{S}^{1} \times \mathbb{R}_{+}$, como

$$
\Phi\left(x_{1}, x_{2}\right)=\left(\left(\frac{x_{1}}{\sqrt{x_{1}^{2}+x_{2}^{2}}}, \frac{x_{2}}{\sqrt{x_{1}^{2}+x_{2}^{2}}}\right), \frac{1}{\sqrt{x_{1}^{2}+x_{2}^{2}}}\right)
$$

onde $\mathbb{R}_{+}$é o conjunto dos números reais positivos.

Observemos que $\Phi$ é um difeomorfismo e que $\Phi\left(\operatorname{Imagem}\left(\gamma^{\prime \prime}\right)\right)=\mathcal{D}_{\widetilde{H}}^{+}$, com isto basta estudar o conjunto Imagem $\left(\gamma^{\prime \prime}\right)$. 
Agora definimos a função $F: I \times \mathbb{R}^{2} \rightarrow \mathbb{R}$ dada pela seguinte relação

$$
F\left(s, x_{1}, x_{2}\right)=x_{1}^{\prime}(s) \operatorname{sen} \mathrm{x}_{1}-\mathrm{x}_{2}^{\prime}(\mathrm{s}) \cos \mathrm{x}_{1}-\mathrm{x}_{2} .
$$

Esta função é uma representação local da função $\widetilde{H}$ numa vizinhança de um ponto em $\mathcal{D}^{+}{ }_{\widetilde{H}}$, por meio de $F\left(s, x_{1}, x_{2}\right)=\widetilde{H}\left(s,\left(\cos x_{1}\right.\right.$, sen $\left.\left.\mathrm{x}_{1}\right), \mathrm{x}_{2}\right)$. Usamos a função $F$ no lugar da função $\widetilde{H}$.

Note que $\left(\cos x_{1}, \operatorname{sen} \mathrm{x}_{1}, \mathrm{x}_{2}\right) \in \mathcal{D}_{\mathrm{H}}$ se, e somente se, $\left(\cos x_{1}, \operatorname{sen} \mathrm{x}_{1}\right)=\left(\lambda(\mathrm{s}) \mathrm{x}_{1}^{\prime \prime}(\mathrm{s}), \lambda(\mathrm{s}) \mathrm{x}_{2}^{\prime \prime}(\mathrm{s})\right)$ e $x_{2}=\lambda(s)$, para algum $s \in I$.

Escrevemos $\gamma(s)=\left(x_{1}(s), x_{2}(s)\right)$ e $u=\left(\cos x_{1}, \operatorname{sen} x_{1}\right)$

$$
\begin{gathered}
\frac{\partial}{\partial x_{1}} F\left(s, x_{1}, x_{2}\right)=x_{1}^{\prime}(s) \cos x_{1}+x_{2}^{\prime}(s) \operatorname{sen} \mathrm{x}_{1} \quad \text { e } \frac{\partial}{\partial \mathrm{x}_{2}} \mathrm{~F}\left(\mathrm{~s}, \mathrm{x}_{1}, \mathrm{x}_{2}\right)=-1 \\
\frac{\partial}{\partial s}\left(\frac{\partial}{\partial x_{1}} F\left(s, x_{1}, x_{2}\right)\right)=x_{1}^{\prime \prime}(s) \cos x_{1}+x_{2}^{\prime \prime}(s) \operatorname{sen} \mathrm{x}_{1} \quad \text { e } \frac{\partial}{\partial \mathrm{s}}\left(\frac{\partial}{\partial \mathrm{x}_{2}} \mathrm{~F}\left(\mathrm{~s}, \mathrm{x}_{1}, \mathrm{x}_{2}\right)\right)=0 .
\end{gathered}
$$

Daí obtemos,

- 0 -jato com constante $\frac{\partial F}{\partial x_{1}}\left(s_{0}, 0,0\right)$ em $s$ é: $\alpha_{0,1}=x_{1}^{\prime}\left(s_{0}\right) \cos x_{1}+x_{2}^{\prime}\left(s_{0}\right) \sin x_{1}$.

- 0 -jato com constante $\frac{\partial F}{\partial x_{2}}\left(s_{0}, 0,0\right)$ em $s$ é: $\alpha_{0,2}=-1$.

- 1-jato com constante $\frac{\partial F}{\partial x_{1}}\left(s_{0}, 0,0\right)$ em $s$ é:

$$
\alpha_{0,1}+\alpha_{1,1} s=x_{1}^{\prime}\left(s_{0}\right) \cos x_{1}+x_{2}^{\prime}\left(s_{0}\right) \operatorname{sen} \mathrm{x}_{1}+\left(\mathrm{x}_{1}^{\prime \prime}\left(\mathrm{s}_{0}\right) \cos \mathrm{x}_{1}+\mathrm{x}_{2}^{\prime \prime}\left(\mathrm{s}_{0}\right) \operatorname{sen} \mathrm{x}_{1}\right) \mathrm{s}
$$

- 1-jato com constante $\frac{\partial F}{\partial x_{2}}\left(s_{0}, 0,0\right)$ em $s$ é: $\alpha_{0,2}+\alpha_{1,2} s=-1+0 s$

Agora vejamos os itens (a) e (b) do item 2 do teorema.

(a) Suponhamos que $\left(\left(\lambda\left(s_{0}\right) x_{1}^{\prime \prime}\left(s_{0}\right), \lambda\left(s_{0}\right) x_{2}^{\prime \prime}\left(s_{0}\right)\right), \lambda\left(s_{0}\right)\right) \in \mathcal{D}_{\widetilde{H}} \quad$ e $\quad \gamma\left(s_{0}\right)$ não é um ponto parabólico de $\gamma$.

Exprimindo $\left(\left(\lambda\left(s_{0}\right) x_{1}^{\prime \prime}\left(s_{0}\right), \lambda\left(s_{0}\right) x_{2}^{\prime \prime}\left(s_{0}\right)\right)\right)=\left(\cos x_{1}, \operatorname{sen}_{1}\right)$ e $\lambda\left(s_{0}\right)=x_{2}$.

Para obter nosso resultado verificamos as condições da Proposiçao (2.29) isto é, verificamos que $H$ é um desdobramento versal de $f$ e $f$ tem singularidade do tipo $A_{1}$.

Afirmação 5. $f$ tem singularidade do tipo $A_{1}$.

$f\left(s_{0}\right)=F_{u}\left(s_{0}\right)=x_{1}^{\prime}\left(s_{0}\right) \operatorname{sen} \mathrm{x}_{1}-\mathrm{x}_{2}^{\prime}\left(\mathrm{s}_{0}\right) \cos \mathrm{x}_{1}-\mathrm{x}_{2}$, substituindo as relações acima, 


$$
\begin{aligned}
& =\lambda\left(s_{0}\right)\left(x_{1}^{\prime}\left(s_{0}\right) x_{2}^{\prime \prime}\left(s_{0}\right)-x_{2}^{\prime}\left(s_{0}\right) x_{1}^{\prime \prime}\left(s_{0}\right)-1\right)=0 \text { pois }\left|\gamma^{\prime}, \gamma^{\prime \prime}\right|=1 . \\
f^{\prime}\left(s_{0}\right) & =F_{u}^{\prime}\left(s_{0}\right)=x_{1}^{\prime \prime}\left(s_{0}\right) \operatorname{sen} \mathrm{x}_{1}-\mathrm{x}_{2}^{\prime \prime}\left(\mathrm{s}_{0}\right) \cos \mathrm{x}_{1}=\lambda\left(\mathrm{s}_{0}\right)\left(\mathrm{x}_{1}^{\prime \prime}\left(\mathrm{s}_{0}\right) \mathrm{x}_{2}^{\prime \prime}\left(\mathrm{s}_{0}\right)-\mathrm{x}_{2}^{\prime \prime}\left(\mathrm{s}_{0}\right) \mathrm{x}_{1}^{\prime \prime}\left(\mathrm{s}_{0}\right)\right)=0 . \\
f^{\prime \prime}\left(s_{0}\right) & =x_{1}^{\prime \prime \prime}\left(s_{0}\right) \operatorname{sen} \mathrm{x}_{1}-\mathrm{x}_{2}^{\prime \prime \prime}\left(\mathrm{s}_{0}\right) \cos \mathrm{x}_{1}=\lambda\left(\mathrm{s}_{0}\right)\left(\mathrm{x}_{1}^{\prime \prime \prime}\left(\mathrm{s}_{0}\right) \mathrm{x}_{2}^{\prime \prime}\left(\mathrm{s}_{0}\right)-\mathrm{x}_{2}^{\prime \prime \prime}\left(\mathrm{s}_{0}\right) \mathrm{x}_{1}^{\prime \prime}\left(\mathrm{s}_{0}\right)\right) \\
& =\lambda\left(s_{0}\right)\left|\gamma^{\prime \prime \prime}\left(s_{0}\right), \gamma^{\prime \prime}\left(s_{0}\right)\right|=-\lambda\left(s_{0}\right) k_{a}\left(s_{0}\right) \neq 0 .
\end{aligned}
$$

Afirmação 6. $H$ é um desdobramento versal de $f$.

Usando o Teorema (2.20), o resultado segue pois, a matriz $1 \times 2$ dos coeficientes $\left(\alpha_{j, i}\right)$ dada por

$$
\left(x_{1}^{\prime}\left(s_{0}\right) \cos x_{1}+x_{2}^{\prime}\left(s_{0}\right) \operatorname{sen} \mathrm{x}_{1}-1\right)
$$

tem posto 1 (Teorema (2.5)).

(b) Suponhamos que $\left(\left(\lambda\left(s_{0}\right) x_{1}^{\prime \prime}\left(s_{0}\right), \lambda\left(s_{0}\right) x_{2}^{\prime \prime}\left(s_{0}\right)\right), \lambda\left(s_{0}\right)\right) \in \mathcal{D}_{\widetilde{H}} \quad$ e $\quad \gamma\left(s_{0}\right)$ é um ponto parabólico de $\gamma$ em $s_{0}$.

Para obter nosso resultado verificamos as condições da Proposiçao (2.29) isto é, verificamos que $H$ é um desdobramento versal de $f$ e que $f$ tem singularidade do tipo $A_{2}$.

Afirmação 7. $f$ tem singularidade do tipo $A_{2}$.

Como $k_{a}\left(s_{0}\right)=0$, então $f^{\prime \prime}\left(s_{0}\right)=0$, e pelo item anterior temos $f\left(s_{0}\right)=f^{\prime \prime}\left(s_{0}\right)=0$. Assim $f$ tem singularidade do tipo $A_{\geq 2}$ e pelas condições de generecidade $\left(M_{1}\right),\left(M_{3}\right)$ e $\left(M_{4}\right), f$ tem singularidade $A_{2}$.

Afirmação 8. $H$ é um desdobramento versal de $f$.

Usando o Teorema (2.20). Verificamos que a matriz $2 \times 2$ dos coeficientes $\left(\alpha_{j, i}\right)$ dada por

$$
\left(\begin{array}{cc}
x_{1}^{\prime}\left(s_{0}\right) \cos x_{1}+x_{2}^{\prime}\left(s_{0}\right) \operatorname{sen} \mathrm{x}_{1} & -1 \\
x_{1}^{\prime \prime}\left(s_{0}\right) \cos x_{1}+x_{2}^{\prime \prime}\left(s_{0}\right) \operatorname{sen} \mathrm{x}_{1} & 0
\end{array}\right),
$$

tem posto 2 isto é que o determinante da matriz é não nulo.

De fato, substituindo

$$
\left(\cos x_{1}, \operatorname{sen} \mathrm{x}_{1}\right)=\frac{\left(\mathrm{x}_{1}^{\prime \prime}\left(\mathrm{s}_{0}\right), \mathrm{x}_{2}^{\prime \prime}\left(\mathrm{s}_{0}\right)\right)}{\sqrt{\left(\mathrm{x}_{1}^{\prime \prime}\left(\mathrm{s}_{0}\right)\right)^{2}+\left(\mathrm{x}_{2}^{\prime \prime}\left(\mathrm{s}_{0}\right)\right)^{2}}}
$$

em $x_{1}^{\prime \prime}\left(s_{0}\right) \cos x_{1}+x_{2}^{\prime \prime}\left(s_{0}\right) \operatorname{sen} \mathrm{x}_{1}$ tem-se,

$$
x_{1}^{\prime \prime}\left(s_{0}\right) \cos x_{1}+x_{2}^{\prime \prime}\left(s_{0}\right) \operatorname{sen} \mathrm{x}_{1}=\sqrt{\left(\mathrm{x}_{1}^{\prime \prime}\left(\mathrm{s}_{0}\right)\right)^{2}+\left(\mathrm{x}_{2}^{\prime \prime}\left(\mathrm{s}_{0}\right)\right)^{2}} \neq 0 .
$$




\section{Referências Bibliográficas}

[1] M. Audin. Geometry. Universitext. Springer-Verlag, Berlin, 2003. Translated from the 1998 French original.

[2] D. A. Brannan, M. F. Esplen, and J. J. Gray. Geometry. Cambridge University Press, Cambridge, 1999.

[3] J. W. Bruce and P. J. Giblin. Curves and singularities. Cambridge University Press, Cambridge, second edition, 1992. A geometrical introduction to singularity theory.

[4] J. W. Bruce, P. J. Giblin, and C. G. Gibson. On caustics of plane curves. Amer. Math. Monthly, 88(9):651-667, 1981.

[5] D. Davis. Generic affine differential geometry of curves in $\mathbb{R}^{n}$. Proc. Roy. Soc. Edinburgh Sect. A, 136(6):1195-1205, 2006.

[6] D. Davis. Affine diferential geometry and singularity theory. PhD thesis, University of Liverpool, April 2008.

[7] D. Davis. Affine normal curvature of hypersurfaces from the point of view of singularity theory. Geom. Dedicata, 141:137-145, 2009.

[8] D. L. Fidal. The existence of sextactic points. Math. Proc. Cambridge Philos. Soc., 96(3):433-436, 1984.

[9] D. L. Fidal and P. J. Giblin. Generic 1-parameter families of caustics by reflexion in the plane. Math. Proc. Cambridge Philos. Soc., 96(3):425-432, 1984.

[10] P. J. Giblin and G. Sapiro. Affine-invariant distances, envelopes and symmetry sets. Geom. Dedicata, 71(3):237-261, 1998.

[11] S. Izumiya and T. Sano. Generic affine differential geometry of plane curves. Proc. Edinburgh Math. Soc. (2), 41(2):315-324, 1998. 
[12] S. Izumiya and T. Sano. Generic affine differential geometry of space curves. Proc. Roy. Soc. Edinburgh Sect. A, 128(2):301-314, 1998.

[13] E. L. Lima. Variedades diferenciáveis, volume 15 of Monografías de Matemática [Mathematical Monographs]. Instituto de Matemática Pura e Aplicada, Rio de Janeiro, 1977.

[14] K. Nomizu and T. Sasaki. Affine differential geometry, volume 111 of Cambridge Tracts in Mathematics. Cambridge University Press, Cambridge, 1994. Geometry of affine immersions.

[15] S. H. Schot. Aberrancy: geometry of the third derivative. Math. Mag., 51(5):259-275, 1978.

[16] S. H. Schot. Geometrical properties of the penosculating conics of a plane curve. Amer. Math. Monthly, 86(6):449-457, 1979.

[17] A. W. Walker. The differential equation of a conic and its relation to the aberrancy. Amer. Math. Monthly, 59:531-538, 1952. 